\title{
Leptin receptor somatic mutations are frequent in HCV-infected cirrhotic liver and associated with hepatocellular carcinoma.
}

\section{$\operatorname{AUTHOR}(S)$ :}

Ikeda, Atsuyuki; Shimizu, Takahiro; Matsumoto, Yuko; Fujii, Yosuke; Eso, Yuji; Inuzuka, Tadashi; Mizuguchi, Aya; ... Uemoto, Shinji; Chiba, Tsutomu; Marusawa, Hiroyuki

\section{CITATION:}

Ikeda, Atsuyuki ... [et al]. Leptin receptor somatic mutations are frequent in HCV-infected cirrhotic liver and associated with hepatocellular carcinoma.. Gastroenterology 2014, 146(1): 222-232.e35

\section{ISSUE DATE:}

2014-01

URL:

http://hdl.handle.net/2433/180778

\section{RIGHT:}

(C) 2014 AGA Institute. Published by Elsevier Inc.; This is not the

published version. Please cite only the published version.; この論文は 出版社版でありません。引用の際には出版社版をご確認ご利用くださ し。 


\title{
Leptin Receptor Somatic Mutations are Frequent in HCV-Infected
}

\section{Cirrhotic Liver and Associate with Hepatocellular Carcinoma}

\author{
Atsuyuki Ikeda ${ }^{1}$, Takahiro Shimizu', Yuko Matsumoto ${ }^{1}$, Yosuke Fujii ${ }^{1}$, \\ Yuji Eso ${ }^{1}$, Tadashi Inuzuka' ${ }^{1}$ Aya Mizuguchi ${ }^{1}$, Kazuharu Shimizu $^{2}$,
}

Etsuro Hatano $^{3}$, Shinji Uemoto ${ }^{3}$, Tsutomu Chiba ${ }^{1}$, and Hiroyuki Marusawa ${ }^{1}$

1. Department of Gastroenterology and Hepatology,

Graduate School of Medicine, Kyoto University, Kyoto, Japan

2. Department of Nanobio Drug Discovery, Graduate School of Pharmaceutical

Sciences, Kyoto University, Kyoto, Japan

3. Department of Surgery, Graduate School of Medicine, Kyoto University, Kyoto, Japan

Corresponding \& Reprint Author: Hiroyuki Marusawa at:

Department of Gastroenterology and Hepatology, Graduate School of Medicine,

Phone; +81-75-751-4319, Fax; +81-75-751-4303

E-mail; maru@kuhp.kyoto-u.ac.jp

Short Title: $L E P R$ mutations in cirrhotic liver

Grant Support: This work was supported by Japan Society for the Promotion of Science (JSPS) Grants-in-aid for Scientific Research, and Health and Labour Sciences Research Grants for Research on Intractable Diseases, and Research on Hepatitis from the Ministry of Health, Labour and Welfare, Japan.

\section{Abbreviations:}


AID: activation-induced cytidine deaminase, HCC: hepatocellular carcinoma, HCV: hepatitis C virus, LEPR: leptin receptor, STAT3: signal transducer and activator of transcription 3, $d b / d b$ mouse: $\mathrm{C} 57 \mathrm{BL} / \mathrm{KsJ}-d b / d b$ mouse, TAA: thioacetamide, Ig: immunoglobulin

Disclosures: The authors have no conflicts of interest.

\section{Data Profiling:}

Sequence reads with Genome Analyzer were deposited in the DNA Data Bank of Japan Sequence Read Archive (http://trace.ddbj.nig.ac.jp/dra/index_e.shtml) under accession number DRA000867.

Author Contributions: A.I. and H.M. designed the study. T.C. and H.M. supervised the project. K.S., E.H., and S.U. provided the material and device of the project. A.I., T.S., Y.M., Y.E., T.I, A.M., Y.E., and H.M. conducted the experiments. A.I., Y.F., and H.M. performed analyses and interpretation of data. A.I., T.C., and H.M. wrote the manuscript. All authors read and contributed to the manuscript 


\begin{abstract}
Background \& Aims: Hepatocellular carcinoma (HCC) develops in patients with chronic hepatitis or cirrhosis via a stepwise accumulation of various genetic alterations. To explore the genetic basis of HCC development in hepatitis C virus (HCV)-associated chronic liver disease, we evaluated genetic variants that accumulate in non-tumor cirrhotic liver.
\end{abstract}

Methods: We determined the whole-exome sequences of 7 tumors and background cirrhotic liver tissues from 4 patients with $\mathrm{HCV}$ infection. We then performed additional sequencing of selected exomes of mutated genes, identified by whole-exome sequencing, and of representative tumor-related genes on samples from 22 cirrhotic livers with $\mathrm{HCV}$ infection. We performed in vitro and in vivo functional studies for 1 of the mutated genes.

Results: Whole-exome sequencing demonstrated that somatic mutations accumulated in various genes in HCV-infected cirrhotic liver tissues. Among the identified genes, the leptin receptor gene $(L E P R)$ was one of the most frequently mutated in tumor and non-tumor cirrhotic liver tissue. Selected exome sequencing analyses detected LEPR mutations in 12 of $22(54.5 \%)$ non-tumorous cirrhotic livers. In vitro, 4 of $7(57.1 \%)$ $L E P R$ mutations found in cirrhotic livers reduced phosphorylation of signal transducer and activator of transcription 3 to inactivate LEPR-mediated signaling. Moreover, $40 \%$ of Lepr-deficient (C57BL/KsJ- $d b / d b)$ mice developed liver tumors following administration of thioacetamide, compared with none of the control mice.

Conclusion: Based on analysis of liver tissues samples from patients, somatic mutations accumulate in LEPR in cirrhotic liver with chronic HCV infection. These mutations could disrupt LEPR signaling and increase susceptibility to hepatocarcinogenesis. 
Keywords: liver cancer; whole exome sequencing; genetics; STAT3 


\section{Introduction}

Chronic inflammation plays an important role in the development of various human cancers. Indeed, many human cancers are closely associated with chronic inflammation, such as Helicobacter pylori-associated gastric cancer and inflammatory bowel disease-associated colorectal cancer ${ }^{1,2}$. On the other hand, tumor cells are thought to be generated by a stepwise accumulation of genetic alterations in various tumor-related genes during the process of inflammation-associated carcinogenesis ${ }^{3-6}$. Thus, it is reasonable to assume that somatic mutations latently accumulate in inflamed tissues where the risk of tumorigenesis is high. Consistent with this hypothesis, several studies demonstrated frequent somatic mutations in non-tumorous inflammatory tissues ${ }^{7,8}$. To clarify the mechanisms of inflammation-associated carcinogenesis, it is important to unveil the genetic alterations that occur in the inflamed tissues before tumor development. The diversity of mutated genes and the low frequency of genetic alterations compared with tumor tissues, however, are obstacles to revealing the landscape of accumulated genetic aberrations in chronically inflamed non-tumorous tissues.

Several possible molecular mechanisms have been proposed for the genetic alterations occurring in the inflammatory condition ${ }^{9}$. We recently demonstrated that the expression of activation-induced cytidine deaminase (AID), a DNA/RNA mutator enzyme family member, links inflammation to an enhanced susceptibility to genetic aberration during the development of various gastrointestinal and hepatobiliary cancers $^{10-12}$. One clear example of inflammation-associated cancer is human hepatocellular carcinoma (HCC). HCC arises in the background of chronic inflammation caused by hepatitis $\mathrm{C}$ virus $(\mathrm{HCV})$ infection ${ }^{13}$. We showed that aberrant AID expression triggered by $\mathrm{HCV}$ infection and the resultant inflammatory response leads to the generation of somatic mutations in various tumor-related genes in the inflamed liver tissues ${ }^{14,15}$. The target genes of AID-mediated mutagenesis in the inflamed hepatocytes, however, remain unclear.

Recent advances in sequencing technology enabled us to reveal the whole picture of human genome sequences in association with the risk of the development of a variety of human diseases, including cancers ${ }^{16}, 17$. Whole exome capture identified several 
candidate driver genes in various human cancers ${ }^{18-20}$. Although deep sequencing on tumor tissues provides the most comprehensive analysis of cancer genome, the genetic alterations accumulated in chronically inflamed tissues might provide an additional opportunity to clarify the early genetic changes required for carcinogenesis. In the present study, we applied whole exome sequencing to not only the tumor but also non-tumorous liver tissues infected with $\mathrm{HCV}$, and found that somatic mutations of the leptin receptor gene $(L E P R)$ latently underlies a subset of the cirrhotic liver tissues, providing the putative genetic basis for $\mathrm{HCV}$-associated hepatocarcinogenesis. 


\section{Materials and Methods}

\section{Whole exome capture and massively-parallel sequencing}

Massively-parallel sequencing was performed as described previously ${ }^{21,} 22$. Fragmented DNA (more than $5 \mu \mathrm{g}$ ) was used to prepare each DNA sequencing library. The DNA libraries were prepared according to the instructions provided with the Illumina Preparation Kit (Illumina, San Diego, CA). Whole exome sequence capture was then performed using SeqCap EZ Human Exome Library v2.0 (Roche, Madison, WI) according to the manufacturer's instructions. Cluster generation was performed on the Illumina cluster station (using their TruSeq PE Cluster Kit v5). Paired-end sequence for $2 \times 76 \mathrm{bp}$ was done on the Illumina Genome Analyzer IIx (using their SBS Kits v5). Data collection and base-calling were performed using SCS v2.9/RTA 1.9 and resultant data files were converted to the FASTQ format.

\section{Selected exome capture and massively-parallel sequencing}

Fragmented DNA $(1 \mu \mathrm{g})$ was used to prepare each DNA sequencing library. The DNA libraries were prepared using TruSeq DNA Sample Prep Kits (Illumina) according to the manufacturer's protocol. Selected gene capture (TP53, CTNNB1, LEPR) was performed using the SeqCap EZ Choice library (Roche) according to the manufacturer's recommendations. Cluster generation and multiplexed paired-end sequencing for $2 \times 71$ $+7 \mathrm{bp}$ was performed as described above. Data collection and base-calling were performed as described above, and demultiplexed using Illumina's CASAVA v1.8.2 software with the default settings.

\section{Sequence data analysis and variant filtering.}

This process is described in Supplemental Information, Supplemental Figure 1, and Supplemental Figure 2.

\section{Patients}

Cell culture and Transfection

Immunoblotting analysis

Animals Experiments

These procedures and information are described in the Supplemental Information. 


\section{Results}

\section{Whole exome sequencing identified the mutation signature of synchronous HCCs in patients with chronic HCV infection.}

To explore the genetic basis of $\mathrm{HCV}$-associated hepatocarcinogenesis, we first determined the whole exome sequences in matched pairs of HCC and background liver tissues obtained from four patients with chronic HCV infection (Supplemental Table 1, \#1-4). Among them, three cases had multiple HCCs and one had a solitary HCC in the liver. To compare the mutation signature in synchronous HCCs that developed in the same background liver, we determined the whole exome sequences of two representative HCCs in three cases and one solitary HCC in the remaining case (Figure 1). These seven HCCs from four patients comprised two well-differentiated and five moderately-differentiated HCCs, and the background liver tissue showed the histologic characteristics of cirrhosis. To subtract the normal variants of each individual from the somatic mutations, we also determined the whole exome sequences of matched peripheral lymphocytes in each patient.

On average, we generated approximately 3.1 gigabases of sequence per sample, $80.1 \%$ of which were aligned with the human reference genome (Human Genome build 37.3), and mean coverage in the targeted regions was 33.8-fold (Supplemental Table 2). The variant filtering process is summarized in Supplemental Figure 1 and the overall error rate in our current platform was confirmed to be less than $0.2 \%$, as described previously $^{21}$. Overall, a total of 970 nucleotide positions in 768 different genes were mutated at a frequency of more than $20 \%$ of reads in the 7 HCC tissues (Supplemental Table 3). Among them, 79 genes were recurrently mutated in two or more tumor tissues (Data not shown). These genes included representative tumor-related genes associated with HCC such as TP53 (mutated in 2/7 tumors). Pathway analyses Kyoto Encyclopedia of Genes and Genomes (KEGG, http://www.genome.jp/kegg/) revealed that metabolic pathway-related genes were most frequently damaged in HCC tissues (5 of 7 tumors) (Supplemental Table 4).

Interestingly, the mutation signature was remarkably different between the synchronously developed HCCs in each individual (Figure 1). In patient \#3, none of the genes were commonly mutated in the two tumors examined, while 29 and 225 genes acquired independent somatic mutations in each tumor, respectively. In contrast, 32 (64.0\% of mutated genes of HCC \#1 in patient \#1) and 9 (24.3\% of mutated genes of HCC \#1 in patient \#2) genes were commonly mutated in the synchronously developed HCCs of those patients, indicating that the synchronous HCCs that developed in patient 
\#1 or \#2 shared a common pattern of genetic aberrations. These findings may suggest that the synchronous tumors in patients \#1 and \#2 were derived from common tumor-precursor cells or developed through intrahepatic metastasis, while the tumors in patient \#3 developed independently in a multicentric manner.

\section{Somatic mutations accumulated in the cirrhotic liver with HCV infection.}

Whole exome sequencing also revealed a large number of nucleotide alterations in the non-tumorous cirrhotic liver tissues. In some cases, the total number of mutated genes in non-tumorous liver was higher than those in tumor tissues, while the mutation frequency in non-tumorous tissues tended to be lower than that in the matched tumor tissues (Figure 2). Sorting Intolerant From Tolerant (SIFT) functional impact predictions (http://provean.jcvi.org/index.php) revealed that the mean percentage of somatic mutations predicted to be "damaging" in tumorous and non-tumorous tissues was $20.4 \%$ and $13.1 \%$, respectively, suggesting that somatic mutations that accumulated in non-tumorous tissues included "passenger" mutations with less functional significance more frequently than those that accumulated in tumor tissues (Supplemental Table 3). We also identified a total of 448 indels in 7 HCC tissues (Supplemental Table 5), while fewer indels were detected in all of the non-tumorous cirrhotic liver tissues examined (Supplemental Table 6). Consistent with previous studies ${ }^{19}$, we found that one-third of the mutations that accumulated in the exome sequences of HCC tissues were enriched as $\mathrm{C}>\mathrm{T}$; $\mathrm{G}>\mathrm{A}$ transition, followed by $\mathrm{A}>\mathrm{G} ; \mathrm{T}>\mathrm{C}$. Similar to tumor tissues, $\mathrm{C}>\mathrm{T}$; G>A transition mutations were most frequently detected in non-tumorous cirrhotic tissues (Supplemental Figure 3).

The aim of this study was to identify the somatic mutations in the non-tumorous HCV-positive cirrhotic liver that may contribute to tumorigenesis. Therefore, we focused on the genes commonly mutated in both tumor and non-tumorous liver tissues of the same individual. Because few genes commonly acquired somatic mutations with a frequency of more than $20 \%$ both in the tumor and the matched non-tumorous liver tissues, we selected potential somatic mutations in non-tumorous tissues that represented more than $5 \%$ of the total reads for further evaluation (Supplemental Figure 1). The 5\% threshold in non-tumorous liver was chosen because common polymorphisms in each individual were excluded by determining the nucleotide changes with a frequency of more than $5 \%$ in the matched normal samples, such as peripheral lymphocytes ${ }^{18,23}$.

Based on these criteria, nucleotide positions that were commonly mutated in both the tumor (at a frequency of $20 \%<$ of reads) and the matched background liver (at a 
frequency of $5 \%<$ of reads) of each patient were detected (Figure 1). Among them, we focused on 40 mutations that result in amino acid changes (Supplemental Table 7), and found that only two genes, LEPR and ZNF408, were recurrently mutated with a frequency greater than 5\% of reads in non-tumorous cirrhotic livers from 2 of the 4 patients (listed as the top two genes of Supplemental Table 7). Of these two genes, we focused on $L E P R$, whose mutations have been correlated with various human diseases, such as obesity and metabolic disorders ${ }^{24}$.

\section{Identification of $L E P R$ as the recurrently mutated gene in the cirrhotic livers with} HCV infection.

We designed a selected sequence capture system that enabled us to enrich the whole exonic sequences of the $L E P R$ followed by deep-sequencing. In addition, selected exonic capture of TP53 and CTNNB1, the representative driver genes for hepatocarcinogenesis ${ }^{19,20,25}$, was also performed on the same cohort. Accordingly, the selected exonic sequencing was applied to 22 additional HCV-positive cirrhotic liver tissues, $10 \mathrm{HCC}$ tissues, and matched peripheral lymphocytes from 22 patients (Supplemental Table 1, \#5-26). Selected exome sequencing generated a mean coverage of 996-, 1656-, and 2348-fold on LEPR, TP53 and CTNNB1, respectively (Supplemental Table 8). The variant filtering process is summarized in Supplemental Figure 2 and we detected both high- (at a frequency of $20 \%<$ of reads) and low- (at a frequency of $1-20 \%$ of reads) frequency mutations separately.

High-frequency mutations in TP53 and CTNNB1 were detectable in 1/10 (10\%) and $1 / 10(10 \%)$ of the HCCs, respectively (Table 1), and these rates in the HCCs were consistent with recent deep-sequencing studies ${ }^{19,20}$. None of the non-tumorous liver tissues possessed high-frequency mutations in TP53 or CTNNB1, however low-frequency mutations of TP53 and CTNNB1 were detected in 17/22 (77.3\%) and $12 / 22(54.5 \%)$ of the non-tumorous livers, respectively. These findings indicated that somatic mutations in the representative cancer driver genes latently accumulated with a relatively low-frequency in the cirrhotic livers with $\mathrm{HCV}$ infection.

Interestingly, we also found high- and/or low-frequency mutations in LEPR in both tumor and non-tumorous liver tissues. Indeed, 9/10 (90\%) tumors and 12/22 (54.5\%) of non-tumorous cirrhotic livers possessed high- and/or low-frequency mutations in LEPR (Table 1). Notably, some somatic mutations were commonly detected in different positions of the same individual's liver. For example, C1084T (Reference position: 65557165) mutations of LEPR were detected in the right-, left-, and caudate lobes of one patient (Supplemental Table 1, \#11), suggesting that some of the hot spots of the 
acquired somatic mutations in the $L E P R$ gene are commonly present in hepatocytes of the same liver underlying HCV infection. On the other hand, deep sequencing of LEPR of non-cirrhotic $\mathrm{HCV}$-associated chronic hepatitis-infected and normal liver tissues revealed no mutations in $L E P R$ of any of the hepatitis-infected or normal livers (Supplemental Table 9). To confirm the somatic mutations present in LEPR in the non-tumorous liver, we validated the candidate mutations by Sanger sequencing. For this purpose, we determined the sequences of exons 9 and 10 of $L E P R$ of at least 50 randomly picked clones that were amplified from the non-tumorous liver tissues of each patient. Although it was difficult to detect all the low-frequency mutations using the conventional cloning-sequencing method, we confirmed that somatic mutations were recurrently accumulated in LEPR of non-tumorous cirrhotic liver tissues (Supplemental Figure 4).

\section{LEPR mutations found in HCV-positive cirrhotic liver resulted in the disruption of downstream signaling.}

Selected exome sequencing detected low-frequency mutations at a total of 650 nucleotide positions of LEPR in 12 of $22(54.5 \%) \mathrm{HCV}$-positive cirrhotic liver tissues. Although the nucleotide changes were unevenly distributed throughout the whole $L E P R$ exonic sequences, we detected 67 nucleotide alterations at the immunoglobulin (Ig) domain of $L E P R, 38$ of which $(56.7 \%)$ were recurrently mutated in two or more patients (Figure 3A). Among them, non-synonymous mutations that caused the amino acid changes were detected at 62 of the $67(92.5 \%)$ nucleotide positions, and 10 of the 62 were also mutated in at least one HCC tissue examined in this study. Histologic examination revealed no significant association between the presence of LEPR mutations and the level of fatty changes in the liver tissue (Data not shown).

To explore the functional relevance of $L E P R$ mutations detected in HCV-positive cirrhotic liver tissues, we randomly selected seven $L E P R$ s with a mutated Ig domain from 62 non-synonymously mutated LEPRs, and examined the downstream signaling properties of the mutated LEPR in vitro.

Accordingly, we subcloned the mutated LEPRs and constructed expression plasmids encoding those mutant LEPRs (Figure 3B). We first confirmed that only a small amount of endogenous LEPR expression was observed in both HEK293 and HepG2 cells (Supplemental Figure 5) and that the induction of the phosphorylation of STAT3 by wild-type $L E P R$ in the presence of recombinant human leptin (Figure 3C). In contrast, four of seven $(57.1 \%)$ mutations in the Ig domain of $L E P R$ resulted in the reduction or loss of STAT3 phosphorylation in vitro (Figure 3C). To clarify the functional 
significance of $L E P R$ mutations, the cell proliferation rate was determined in HepG2 cells expressing either wild-type or mutated LEPRs that were identified in HCV-positive cirrhotic liver tissues using the lentivirus system ${ }^{26}$. Upregulation of cyclin D1 and/or E transcripts as well as enhanced cell proliferation were observed in the cells with expression of the mutated $L E P R$ gene compared with wild-type cells, while there was no difference in the expression levels and subcellular localization between wild-type and mutated LEPR protein (Supplemental Figure 6). These findings indicate that some of the somatic mutations that latently accumulated in the Ig domain of $L E P R$ of the cirrhotic liver tissue might cause dysfunction of LEPR-mediated signaling in the cells with those somatic mutations.

\section{LEPR dysfunction enhanced susceptibility to tumorigenesis.}

To determine the functional relevance of LEPR dysfunction on liver cancer development, we examined whether disruption of the LEPR gene contributes to liver tumorigenesis using a genetically altered mouse model, Lepr-deficient C57BL/KsJ- $d b / d b$ mouse ( $d b / d b$ mouse $)^{27}$. Thioacetamide (TAA), a putative carcinogen, is well established to induce liver fibrosis and tumorigenesis in a murine $\operatorname{model}^{28}$. Thus, we conducted an assay to evaluate whether LEPR insufficiency alters the effects of TAA-mediated tumorigenesis. Accordingly, TAA was prepared at a concentration of $0.02 \%$, a relatively low dose compared to carcinogenic dose ${ }^{29}$, and administered to mice in the drinking water for 24 weeks. The body weight of the $d b / d b$ mice was about twice that of their lean littermates, and $d b / d b$ mice had hepatomegaly even after normalizing the liver weight to the body weight (Figure 4A). Histologic examination revealed the accumulation of lipid within individual hepatocytes in the $d b / d b$ mouse liver, a typical feature of steatosis (Figure 4A).

After administering TAA, the blood levels of alanine aminotransferase were substantially elevated in $d b / d b$ mice compared with those of control mice (Supplemental Table 10). Consistently, histologic examination revealed that inflammatory activity was more severe in the liver of $d b / d b$ mice than that in the liver of control mice (Figure 4B). None of the control mice receiving TAA treatment showed tumorigenesis 24 weeks after TAA administration. In contrast, macroscopic liver nodules developed in 4 of 10 (40\%) $d b / d b$ mice that received the same dose of TAA during the same observation period (Table 2). Histologic examination revealed that two $d b / d b$ mice with liver nodules developed well-differentiated HCC (Figure 4C). In addition, the remaining nodules that developed in $d b / d b$ mouse liver showed features of hepatocyte hyperplasia. These findings suggest that Lepr-deficient $d b / d b$ mice had high susceptibility to TAA-induced 
liver tumorigenesis. 


\section{Discussion}

Tumor cells are considered to be generated by a stepwise accumulation of genetic alterations in tumor-related genes during the process of inflammation-associated carcinogenesis. Several studies have reported that epithelial tissues exposed to chronic inflammation accumulate genetic alterations in tumor-related genes before the onset of tumorigenesis $^{7,8}$. Given that chronic inflammation induces somatic mutations, it is reasonable to assume that critical genetic alterations that contribute to tumorigenesis might emerge in chronically inflamed epithelial cells. Using whole exome sequencing, we demonstrated here that considerable levels of somatic mutations accumulate not only in tumors but also in the non-tumorous liver of patients with $\mathrm{HCV}$-related cirrhosis.

Whole exome sequencing on synchronously developed HCCs demonstrated a remarkable difference in the mutation signature in each case. In two cases, more than $20 \%$ of the mutated genes were commonly present in two tumors that developed in the same background liver, suggesting that these tumors were derived from a common origin or developed through intrahepatic metastasis. In contrast, the tumors that developed in the remaining case shared no common mutations, suggesting independent development in a multicentric manner. The data obtained from the latter case are consistent with those of a recent study in which no common somatic mutations were identified in the two pairs of multicentric HCCs that developed in the same background livers $^{19}$. Taken together, these findings suggest that comprehensive whole exome sequencing on synchronously developed HCCs would permit distinction of the carcinogenic process between tumors that develop in a multicentric manner and that develop through intrahepatic metastasis.

Interestingly, we found that in some cases the total number of mutated genes of non-tumorous liver tissues was larger than those of the matched tumor tissues, possibly due to the abundance of heterogeneous accumulation of passenger mutations in the non-tumorous liver tissues ${ }^{30}$. The observation that the frequency of mutations at each nucleotide position in the non-tumorous tissues tended to be lower than those in the matched tumor tissues may lend support to such a possibility. Notably, somatic mutations in the representative tumor-related genes, TP53 and CTNNB1, were also latently accumulated in the cirrhotic liver tissues. It is unknown whether the TP53 and/or CTNNB1 mutations detected in non-tumorous tissues were derived from the clinically-undetectable small nest of cancer cells or premalignant hepatocytes, however, it is possible that these latent genetic alterations in tumor driver genes contribute to the development of HCC in the background of chronic liver disease. 
Among the various mutated genes in the cirrhotic liver tissue, we identified $L E P R$ as the one of the most recurrently mutated genes. Indeed, we confirmed a total of 650 low-frequency mutations of the LEPR gene in 12 of $22(54.5 \%)$ patients with HCV infection by using selected exome sequencing. At present, it is not clear why a large number of mutations accumulate in the $L E P R$ gene of non-tumorous cirrhotic liver in patients with chronic $\mathrm{HCV}$ infection. One possibility may be that the $L E P R$ gene is highly sensitive to AID-mediated mutagenesis in hepatocytes, because we recently observed that AID activation in cultured hepatoma-derived cells preferentially caused somatic mutations in the LEPR gene (Supplemental Table 11). On the other hand, close attention must be paid to the fact that only low-frequency mutations were detected in the $L E P R$ gene in tumor tissues, consistent with the reported cancer genome database (ICGC dataset version12; http://dcc.icgc.org/web/). In general, tumor-specific driver mutations in tumor tissues are characterized by the high frequency mutations (e.g., $20 \%<$ nucleotide changes of total reads ${ }^{18,23,31}$ ). In this regard, the frequency of any mutation in the LEPR gene observed in the tumor tissues was less than $20 \%$ in our cases. Thus, the genetic changes in LEPR are unlikely to be direct driver mutations for HCC, but rather might play some role in the development of HCC in HCV-infected inflamed liver by providing a pathophysiologic background for hepatocarcinogenesis by modifying the cell proliferation activity.

Leptin is a circulating hormone secreted by adipocytes and regulates energy homeostasis $^{32}$. Leptin acts through binding to the extracellular domain of specific membrane receptor LEPR, which belongs to a family of class I cytokine receptors ${ }^{33}$. The extracellular domain of LEPR comprises two canonical cytokine receptor homology domains, Ig and fibronectin III domains, and the Ig domain is essential for the formation of the hexameric complex and for receptor activation ${ }^{34}$. In the present study, we confirmed that 67 mutations were present in the Ig domain of $L E P R$ in cirrhotic liver, and more than half of the mutations were recurrently mutated in two or more patients. Notably, more than $90 \%$ of those nucleotide alterations that accumulated in the Ig domain of $L E P R$ were non-synonymous mutations. Furthermore, we revealed that several non-synonymous mutations that appeared in the $\operatorname{Ig}$ domain of LEPR impaired signaling to STAT3 in response to leptin, causing the dysregulation of leptin signaling in the cells with those mutations. Sequencing the LEPR gene in patients with severe early onset obesity revealed that the extracellular region of the LEPR has a variety of mutations in those patients ${ }^{35}$. A functional study of missense mutations in the $L E P R$ found in severely obese patients also revealed that mutated LEPR has impaired signaling to STAT3, which is consistent with their inability to activate pathways 
involved in the reduction of food intake ${ }^{36}$. Together, these findings suggested that somatic mutations in the LEPR gene might provide the genetic basis for developing metabolic dysregulation in hepatocytes during hepatocarcinogenesis.

In the present study, we demonstrated for the first time that $d b / d b$ mice with disruption of the Lepr gene were more susceptible to developing hepatic inflammation as well as TAA-mediated tumorigenesis than wild-type mice. Consistent with our findings, a previous study reported an increased incidence of hepatocyte hyperplasia in leptin-deficient $o b / o b$ mice, a model for nonalcoholic fatty liver disease ${ }^{37}$. Taken together, it is strongly suggested that dysregulation of LEPR signaling has a role in hepatic tumor development, but the mechanism of how the leptin signaling deficiency contributes to an enhanced inflammatory response and tumorigenesis is currently unknown. It should be noted that both $o b / o b$ mice and $d b / d b$ mice are characterized by hepatic steatosis, and steatosis is well recognized as a common histopathologic feature of the chronic $\mathrm{HCV}$-infected liver. Epidemiologic studies revealed that fatty liver disease may be a common underlying pathology in patients with $\mathrm{HCC}^{38,39}$, and steatosis is an important cofactor in accelerating the development of hepatic fibrosis and inflammatory activity ${ }^{40,41}$, contributing to the progression of HCC in HCV-related chronic liver disease ${ }^{42}$. In the present study, we found no correlation between the prevalence of $L E P R$ mutations and the histologic feature of fatty changes in $\mathrm{HCV}$-positive cirrhotic liver tissues. On the other hand, previous studies demonstrated that leptin can oppose the action of insulin-induced signaling by reducing the phosphorylation of insulin receptor substrate- 1 in human hepatic cells ${ }^{43,44}$. In addition, it was shown that leptin suppresses $\mathrm{HCC}$ via activation of the immune response, suggesting the tumor-suppressing function of leptin-mediated signaling ${ }^{45}$. Thus, we speculate that dysregulation of leptin signaling in the liver might be involved in the neoplastic process of patients with HCV-related chronic liver damage. Because somatic mutations in $L E P R$ are limited to a small proportion of cells in cirrhotic liver tissue and the TAA-mediated liver inflammation model does not fully recapitulate $\mathrm{HCV}$-associated chronic liver disease, further analysis is required to determine whether dysregulation of LEPR-mediated signaling caused by LEPR mutations contributes to the enhanced inflammatory response or tumorigenesis in patients with HCV-related chronic liver damage.

In conclusion, we showed that various somatic mutations latently accumulate in the non-tumorous cirrhotic liver of patients with $\mathrm{HCV}$ infection. The findings that the LEPR gene was recurrently mutated in cirrhotic liver provide a novel putative link between the inflammation-mediated genetic aberrations, the dysregulation of leptin-signaling, and 
the development of HCC in patients with HCV-related chronic liver disease. The gene catalogue identified in the HCV-infected chronically damaged liver might contain the putative driver gene associated with tumor initiation as well as the gene that provides the genetic basis for the development of HCC. Thus, further studies are required to identify the genetic alterations that contribute to tumor development in chronically inflamed liver underlying chronic HCV infection.

\section{Acknowledgements}

We thank Dr. K. Terasawa, Dr. T. Fujiwara, Dr. K. Takahashi, and Dr. Y. Ueda for helpful suggestion and Dr. N. Nishijima, Dr. A. Takai, Dr. A. Nasu, Dr. Y. Endo, Ms. Y. Nakagawa, and Ms. C. Kakimoto for help with the analyses. And we specially thank Dr. H. Kokuryu and Dr. T. Kusaka at Kyoto Katsura Hospital for great support. 


\section{Figure legends}

Fig. 1 Schematic diagram showing the number of mutated genes in tumors, and the number of genes commonly mutated in both tumor and the matched non-tumorous liver tissues.

Patients \#1, \#2 and \#3 had synchronously developed HCCs, and patient \#4 had a solitary HCC. Venn diagrams represent the number of mutated genes in each HCC tissue determined by whole exome sequencing. The numbers of genes commonly mutated in the synchronously developed multiple HCCs were 32,9 , and 0 in patients \#1, $\# 2$, and \#3, respectively. Among the mutated genes in HCC (at a frequency of $20 \%<$ of reads), the number of genes commonly mutated in both HCC and matched non-tumorous background liver (at a frequency of $5 \%<$ of reads) is shown in shaded circles.

Fig. 2 Number of mutated genes and the distribution of mutation frequency in tumor and non-tumorous cirrhotic liver tissues.

The number of mutated genes (upper) and the distribution of mutation frequency (lower) detected by whole exome sequencing in each sample are shown (at a frequency of $20 \%<$ of reads). Patients \#1, \#2, and \#4 had more mutated genes in non-tumorous liver tissue than those in $\mathrm{HCC}$, while the mutation frequency at each nucleotide position in the majority of non-tumorous cirrhotic liver tissues was less than $30 \%$. NT denotes non-tumorous cirrhotic liver.

Fig. 3 Distribution of mutations in the $L E P R$ sequence in HCV-positive cirrhotic liver tissues.

(A) Schematic diagram of the LEPR gene (top) and the immunoglobulin (Ig) domain (middle). Mutated positions in the Ig domain are indicated by the black triangles. A total of 38 of 67 (56.7\%) mutated nucleotide positions of the Ig domain were recurrently mutated in two or more HCV-positive cirrhotic liver tissues. Frequencies of non-synonymous (black circle) and synonymous (gray diamond) mutations at each nucleotide position of the Ig domain of each sample are shown (bottom). Non-synonymous mutations were detected at 62 of the 67 nucleotide positions.

(B)(C) HEK293 cells were transfected with constructs encoding wild-type or representative various mutated $L E P R$ s that were identified in $\mathrm{HCV}$-positive cirrhotic liver tissues. Control: empty vector (B) Immunoblotting was performed on the lysate of 
the cells expressing either wild-type or a mutated Ig domain (D332Y, V333A, Y426X, and V427M) of the LEPR gene using anti-Myc antibodies. (C) After transfection, the cells were treated with or without recombinant leptin protein. Total protein was isolated and immunoblot analysis was performed using anti-phospho-STAT3 (upper panels) and anti-total-STAT3 (lower panels).

Fig. 4 Tumors developed in $d b / d b$ mice treated with thioacetamide (TAA).

(A) Representative macroscopic (left) and microscopic (right) images (hematoxylin and eosin (H\&E) stain) of the liver from a $d b / d b$ mouse and a littermate control mouse without TAA administration. The liver of the $d b / d b$ mouse is enlarged and yellowish compared with the control (yellow arrowheads). Histologic analysis of the liver tissue of $d b / d b$ (ii, iv) and control (i, iii). (original magnification, $4 \times$ for upper panels, $10 \times$ for lower panels)

(B) Microscopic images (H\&E stain) of control (i, iii) and $d b / d b$ mice (ii, iv) with TAA for 24 weeks. In the $d b / d b$ mice, inflammatory cell infiltration was extensively observed in the liver tissues underlying prominent steatosis (ii, iv). (original magnification, $4 \times$ for upper panels, $20 \times$ for lower panels)

(C) Microscopic images (H\&E stain) of nodules that developed in $d b / d b$ mice treated with TAA for 24 weeks (i-vi). Liver cancers developed in two $d b / d b$ mice (i, ii and iii, iv). Arrowheads indicate hepatocyte hyperplasia (v, vi). (original magnification, $4 \times$ for left panels, $10 \times$ for right panels) 


\section{Reference}

1. Coussens LM, Werb Z. Inflammation and cancer. Nature 2002;420:860-7.

2. Chiba T, Marusawa H, Ushijima T. Inflammation-associated cancer development in digestive organs: mechanisms and roles for genetic and epigenetic modulation. Gastroenterology 2012;143:550-63.

3. Lengaver C, Kinzler KW, Vogelstein B. Genetic instabilities in human cancers. Nature 1998:396:643-9.

4. Hanahan D, Weinberg RA. The hallmarks of cancer. Cell 2000;100:57-70.

5. Hussain SP, Schwank J, Staib F, et al. TP53 mutations and hepatocellular carcinoma: insights into the etiology and pathogenesis of liver cancer. Oncogene 2007;26:2166-76.

6. Loeb LA, Bielas JH, Beckman RA. Cancers exhibit a mutator phenotype: clinical implications. Cancer Res 2008;68:3551-7.

7. Barrett MT, Sanchez CA, Prevo LJ, et al. Evolution of neoplastic cell lineages in Barrett oesophagus. Nat Genet 1999;22:106-9.

8. Leedham SJ, Graham TA, Oukrif D, et al. Clonality, founder mutations, and field cancerization in human ulcerative colitis-associated neoplasia. Gastroenterology 2009;136:542-50.

9. Hussain SP, Hofseth LJ, Harris CC. Radical causes of cancer. Nat Rev Cancer 2003;3:276-85.

10. Matsumoto $\mathrm{Y}$, Marusawa H, Kinoshita K, et al. Helicobacter pylori infection triggers aberrant expression of activation-induced cytidine deaminase in gastric epithelium. Nat Med 2007;13:470-6.

11. Komori J, Marusawa H, Machimoto $\mathrm{T}$, et al. Activation-induced cytidine deaminase links bile duct inflammation to human cholangiocarcinoma. Hepatology 2008;47:888-96.

12. Endo $\mathrm{Y}$, Marusawa $\mathrm{H}, \mathrm{Kou} \mathrm{T}$, et al. Activation-induced cytidine deaminase links between inflammation and the development of colitis-associated colorectal cancers. Gastroenterology 2008;135:889-98.

13. Ikeda K, Marusawa H, Osaki Y, et al. Antibody to hepatitis B core antigen and risk for hepatitis C-related hepatocellular carcinoma: a prospective study. Ann Intern Med 2007;1 46:649-56.

14. Endo Y, Marusawa H, Kinoshita K, et al. Expression of activation-induced cytidine deaminase in human hepatocytes via NF-kappaB signaling. Oncogene 2007;26:5587-95. 
15. Kou T, Marusawa H, Kinoshita K, et al. Expression of activation-induced cytidine deaminase in human hepatocytes during hepatocarcinogenesis. Int J Cancer 2007;120:469-76.

16. Wei X, Walia V, Lin JC, et al. Exome sequencing identifies GRIN2A as frequently mutated in melanoma. Nat Genet 2011 :43:442-6.

17. Wang L, Tsutsumi S, Kawaguchi T, et al. Whole-exome sequencing of human pancreatic cancers and characterization of genomic instability caused by MLHI haploinsufficiency and complete deficiency. Genome Res 2012;22:208-19.

18. Wang K, Kan J, Yuen ST, et al. Exome sequencing identifies frequent mutation of ARIDIA in molecular subtypes of gastric cancer. Nat Genet 201 1;43:1219-23.

19. Fujimoto A, Totoki $Y$, Abe $\mathrm{T}$, et al. Whole-genome sequencing of liver cancers identifies etiological influences on mutation patterns and recurrent mutations in chromatin regulators. Nat Genet 2012;44:760-4.

20. Guichard C, Amaddeo G, Imbeaud S, et al. Integrated analysis of somatic mutations and focal copy-number changes identifies key genes and pathways in hepatocellular carcinoma. Nat Genet 2012;44:694-8.

21. Nasu A, Marusawa H, Ueda Y, et al. Genetic heterogeneity of hepatitis $C$ virus in association with antiviral therapy determined by ultra-deep sequencing. PLoS One 2011;6:e24907.

22. Nishijima N, Marusawa H, Ueda Y, et al. Dynamics of hepatitis B virus quasispecies in association with nucleos(t)ide analogue treatment determined by ultra-deep sequencing. PLoS One 2012;7:e35052.

23. Varela I, Tarpey $P$, Raine $K$, et al. Exome sequencing identifies frequent mutation of the SWI/SNF complex gene PBRM1 in renal carcinoma. Nature 2011;469:539-42.

24. Clément $\mathbf{K}$, Vaisse $\mathbf{C}$, Lahlou $\mathbf{N}$, et al. A mutation in the human leptin receptor gene causes obesity and pituitary dysfunction. Nature 1998;392:398-401.

25. Laurent-Puig P, Zucman-Rossi J. Genetics of hepatocellular tumors. Oncogene 2006:25:3778-86.

26. Morita S, Matsumoto Y, Okuyama S, et al. Bile acid-induced expression of activation-induced cytidine deaminase during the development of Barrett's oesophageal adenocarcinoma. Carcinogenesis 2011;32:1706-12.

27. Lee $\mathrm{GH}$, Proenca R, Montez JM, et al. Abnormal splicing of the leptin receptor in diabetic mice. Nature 1996;379:632-5.

28. Becker FF. Thioacetamide hepatocarcinogenesis. J Natl Cancer Inst 1983;71:553-8. 
29. Schnur J, Nagy P, Sebestyén A, et al. Chemical hepatocarcinogenesis in transgenic mice overexpressing mature TGF beta-1 in liver. Eur J Cancer 1999;35:1842-5.

30. Greenman C, Stephens P, Smith R, et al. Patterns of somatic mutation in human cancer genomes. Nature 2007:446:153-8.

31. Yan XJ, Xu J, Gu ZH, et al. Exome sequencing identifies somatic mutations of DNA methyltransferase gene DNMT3A in acute monocytic leukemia. Nat Genet 2011;43:309-15.

32. Schwartz MW, Woods SC, Porte D, et al. Central nervous system control of food intake. Nature 2000;404:661-71.

33. Tartaglia LA. The leptin receptor. J Biol Chem 1997;272:6093-6.

34. Peelman F, Iserentant $\mathbf{H}$, De Smet AS, et al. Mapping of binding site III in the leptin receptor and modeling of a hexameric leptin.leptin receptor complex. J Biol Chem 2006;281:15496-504.

35. Farooqi IS, Wangensteen T, Collins $S$, et al. Clinical and molecular genetic spectrum of congenital deficiency of the leptin receptor. $N$ Engl J Med 2007;356:237-47.

36. Kimber $\mathbf{W}$, Peelman $\mathbf{F}$, Prieur $X$, et al. Functional characterization of naturally occurring pathogenic mutations in the human leptin receptor. Endocrinology 2008;149:6043-52.

37. Yang $\mathrm{S}$, Lin $\mathrm{HZ}$, Hwang J, et al. Hepatic hyperplasia in noncirrhotic fatty livers: is obesity-related hepatic steatosis a premalignant condition? Cancer Res 2001;61:5016-23.

38. Marrero JA, Fontana RJ, SU GL, et al. NAFLD may be a common underlying liver disease in patients with hepatocellular carcinoma in the United States. Hepatology 2002;36:1349-54.

39. Kodama Y, Brenner DA. C-Jun N-terminal kinase signaling in the pathogenesis of nonalcoholic fatty liver disease: Multiple roles in multiple steps. Hepatology 2009:49:6-8.

40. Hourigan LF, Macdonald GA, Purdie D, et al. Fibrosis in chronic hepatitis C correlates significantly with body mass index and steatosis. Hepatology 1999;29:1215-9.

41. Lonardo A, Adinolfi LE, Loria P, et al. Steatosis and hepatitis $C$ virus: mechanisms and significance for hepatic and extrahepatic disease. Gastroenterology 2004;126:586-97.

42. Ohata K, Hamasaki K, Toriyama K, et al. Hepatic steatosis is a risk factor for 
hepatocellular carcinoma in patients with chronic hepatitis $C$ virus infection. Cancer 2003:97:3036-43.

43. Cohen B, Novick D, Rubinstein M. Modulation of insulin activities by leptin. Science 1996;274:1185-8.

44. Wang $Y$, Kuropatwinski KK, White DW, et al. Leptin receptor action in hepatic cells. J Biol Chem 1997;272:16216-23.

45. Elinav E, Abd-Elnabi A, Pappo O, et al. Suppression of hepatocellular carcinoma growth in mice via leptin, is associated with inhibition of tumor cell growth and natural killer cell activation. J Hepatol 2006;44:529-36.

Author names in bold designate shared co-first authorship. 


\section{Supplemental Figure 1. Ikeda et al.}

\section{Whole exome sequencing}

[variant filtering process]

Identification of somatic mutations

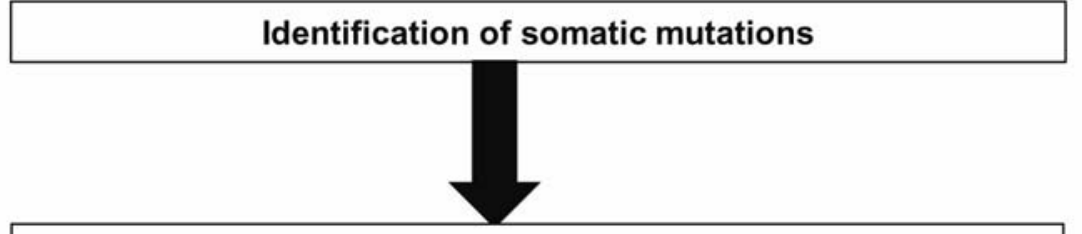

Criteria for selection of somatic mutations

- The overall mutation score must be $\geqq 7$

- The mismatch score must be $\geqq 1$

- The wrong allele score must be $\geqq 0.95$

- Coverage must be $\geqq 11$

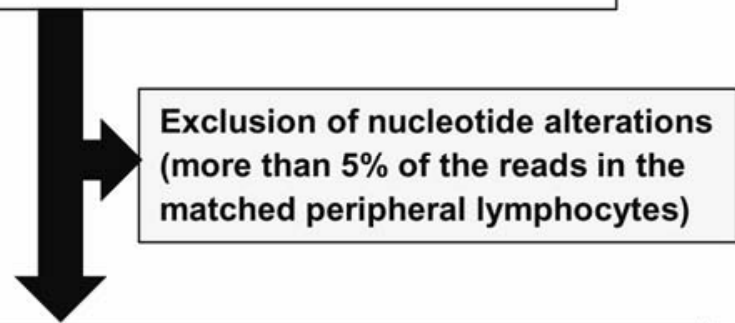

Nucleotide alteration rate $20 \%<$

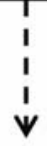

Criteria for selection of the genes commonly mutated in both tumor and non-tumorous liver tissues

In non-tumorous liver tissues:

Nucleotide alteration rate $5 \%<$ (coverage $21 \leqq$ ) 


\section{Supplemental Figure 2. Ikeda et al.}

\section{Selected exome sequencing}

[variant filtering process]
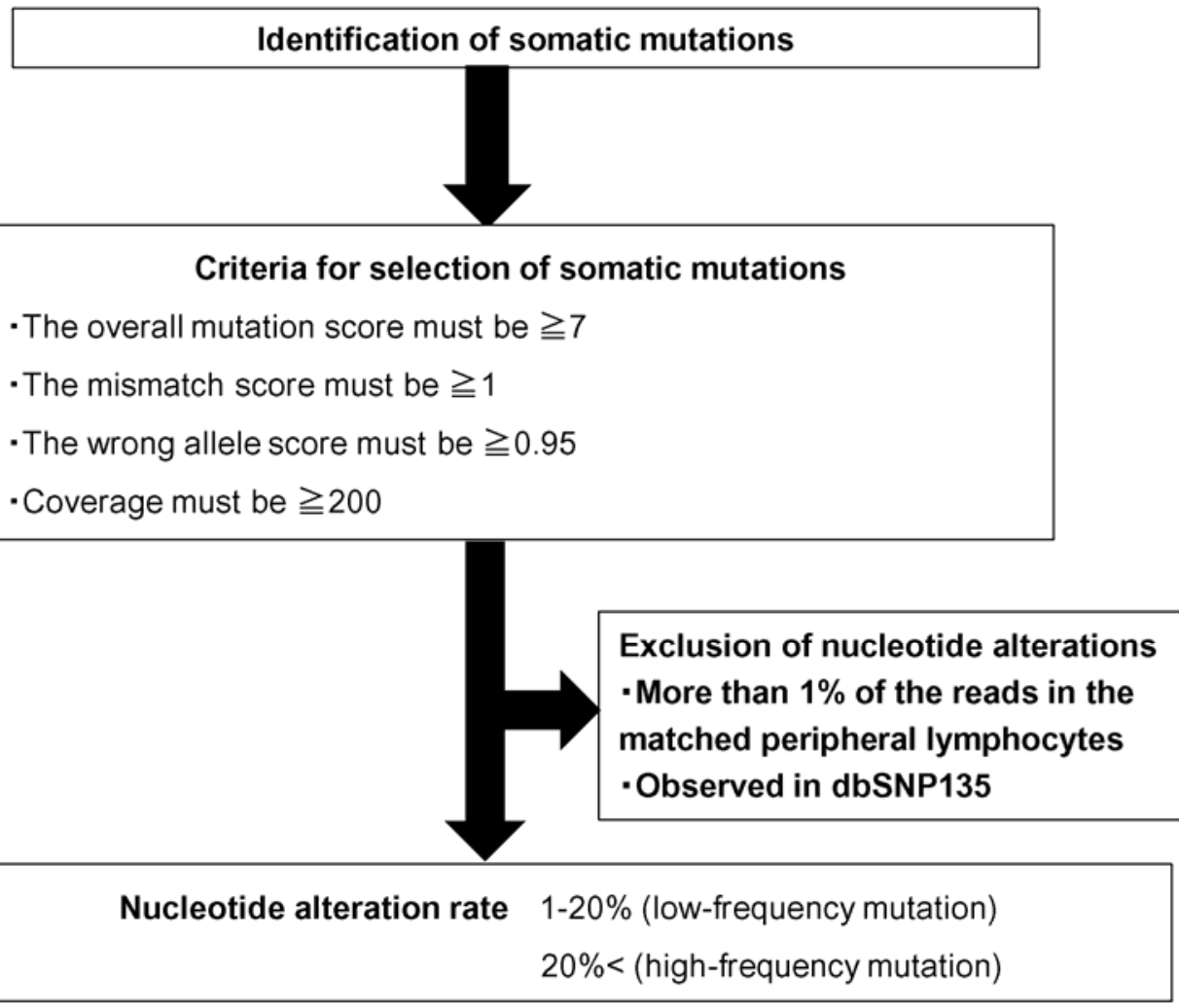

Nucleotide alteration rate $1-20 \%$ (low-frequency mutation) $20 \%<$ (high-frequency mutation) 
Supplemental Figure 3. Ikeda et al.

\section{tumor tissue}

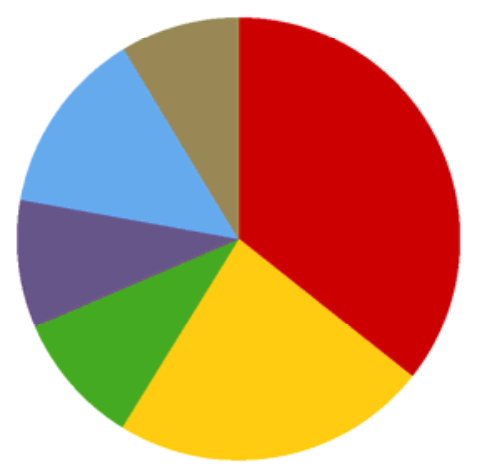

\section{non-tumorous tissue}

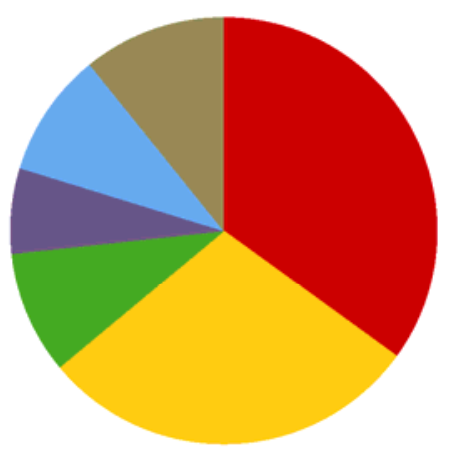

$\because \mathrm{C}>\mathrm{T} ; \mathrm{G}>\mathrm{A}\|\mathrm{A}>\mathrm{G} ; \mathrm{T}>\mathrm{C}\| \mathrm{A}>\mathrm{C} ; \mathrm{T}>\mathrm{G}\|\mathrm{A}>\mathrm{T} ; \mathrm{T}>\mathrm{A}\| \mathrm{C}>\mathrm{A} ; \mathrm{G}>\mathrm{T} \| \mathrm{C}>\mathrm{G} ; \mathrm{G}>\mathrm{C}$

Supplemental Figure 3. Mutation patterns of tumorous and non-tumorous tissues. Mutation signature in seven HCC tumor tissues (upper) and four non-tumorous cirrhotic liver tissues (lower) detected by whole exome sequencing. 


\section{Supplemental Figure 4. Ikeda et al.}

Supplemental Figure 4. Representative Sanger sequencing results of LEPR. Comparison of the sequenced regions between the matched control samples derived from the lymphocytes (upper) and mutated clones derived from non-tumorous cirrhotic liver tissues (lower) of patients with chronic HCV infection. Mutated positions are indicated by red arrows. Amino acid changes are shown in parentheses.

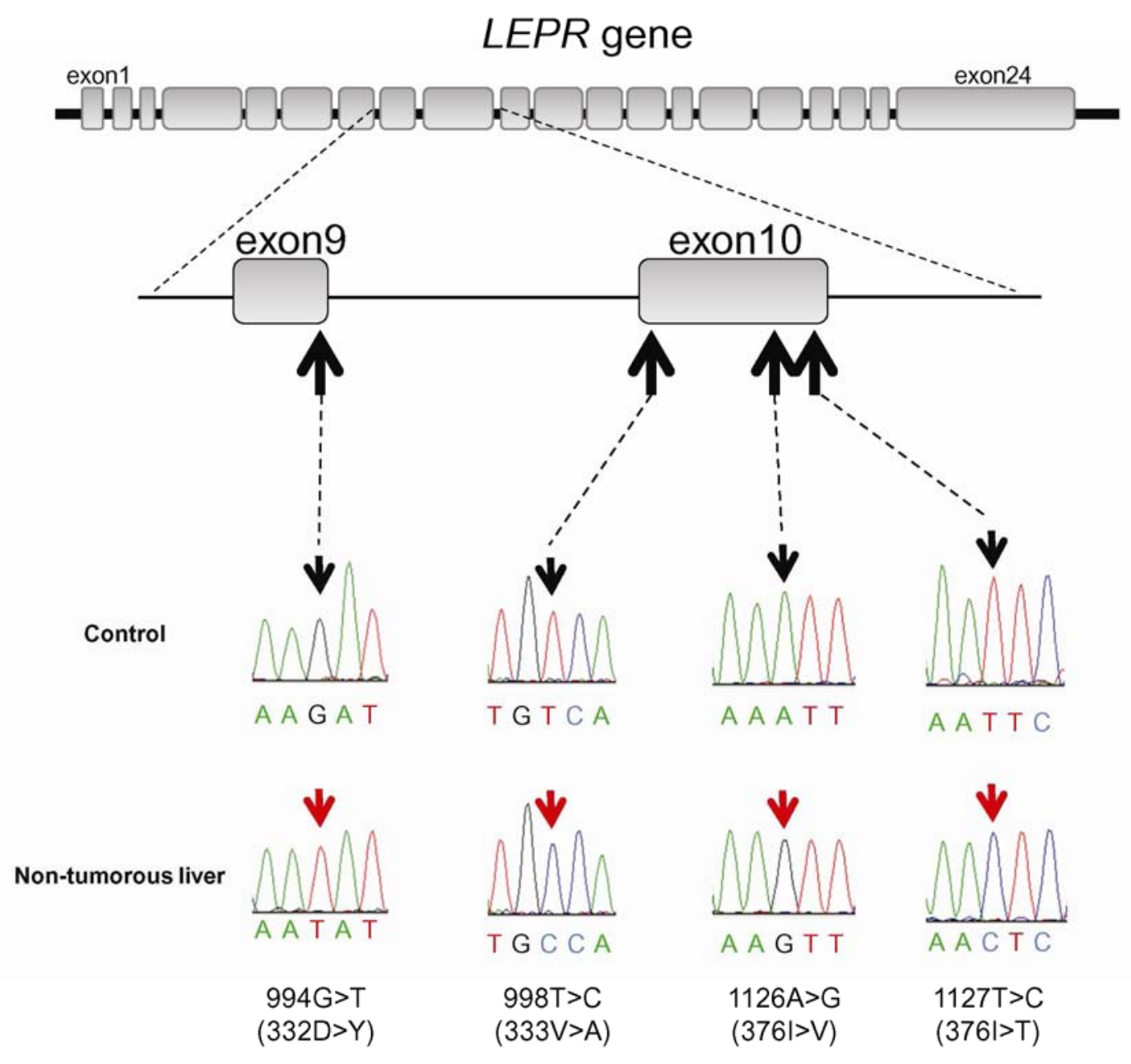


Supplemental Figure 5. Ikeda et al.

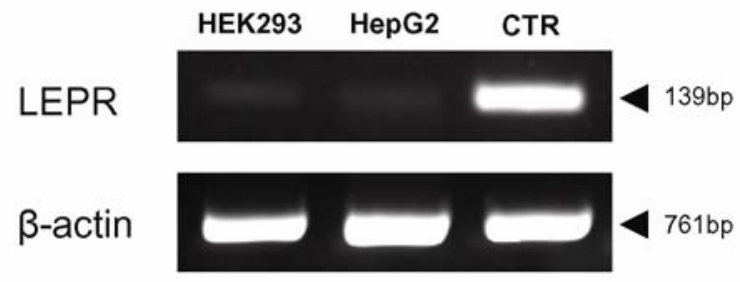

Supplemental Figure 5. Expression of endogenous LEPR in HEK293 and HepG2 cells detected by semi-quantitative RT-PCR analysis. CTR (control) is HEK293 cells transfected with constructs encoding wild-type LEPR as positive control. 


\section{Supplemental Figure 6. Ikeda et al.}

A

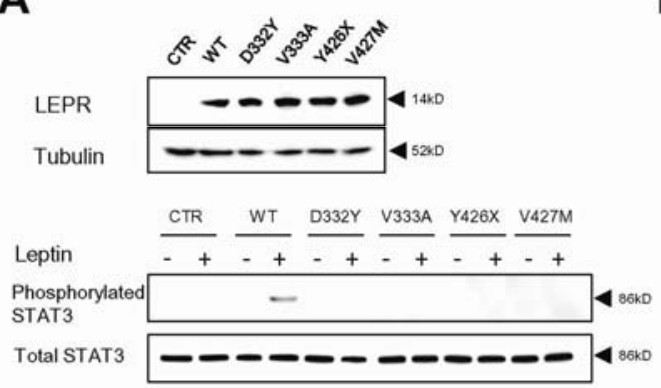

C

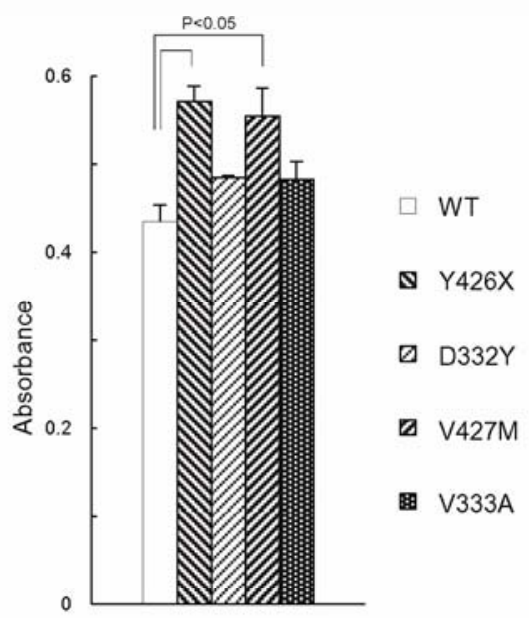

B
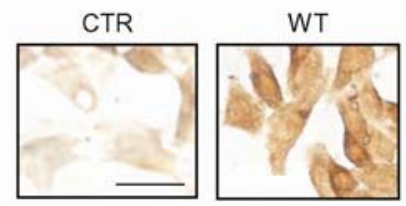

D332Y

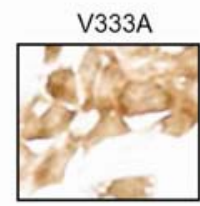

Y426X

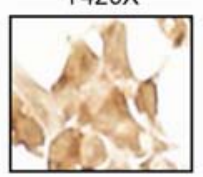

V427M

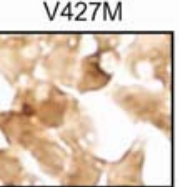

D

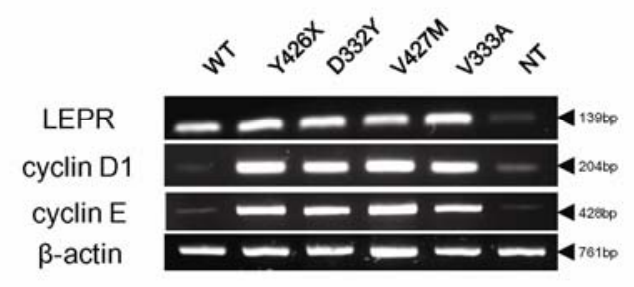

Supplemental Figure 6. Effect of wild-type or mutated LEPR expression on the cell proliferation of HepG2 cells. Expression of either wild-type or mutant LEPRs in HepG2 cells was induced using a lentivirus system ${ }^{26}$. LEPR complementary DNA fragments were inserted into the viral vectors, followed by the production of lentiviral stocks in HEK293 cells. HepG2 cells were cultured in virus-containing medium for $48 \mathrm{~h}$, serum starved for $8 \mathrm{~h}$, treated with $100 \mathrm{ng} / \mathrm{mL}$ recombinant human leptin (SigmaAldrich) for 10min, and then subjected to immunoblotting, immunostaining, semi-quantitative RT-PCR, or cell proliferation (MTT) assay. (A) Immunoblotting was performed on the lysate of the cells expressing wild-type or mutated Ig domain (D332Y, V333A, Y426X, and V427M) of the LEPR gene using anti-Myc antibodies. (B) Representative immunostaining for wild-type and mutated LEPRs (Y426X, D332Y, V427M, and V333A) are shown. Immunohistochemistry using specific antibodies for human LEPR (Ob-R (C-20), Santa Cruz Biotechnology, Inc., Dallas, TX) was performed on HepG2 cells expressing various $L E P R$ constructs. Scale bar $=10 \mu \mathrm{m}$. (C) Proliferation activities of HepG2 cells expressing various $L E P R$ constructs were evaluated by MTT assay. Equal numbers $\left(1 \times 10^{4}\right)$ of the cells were cultured in a 96-well culture plate, followed by the expression of the various LEPR constructs. At $24 \mathrm{~h}$ post-transfection, the cells were serum starved for $48 \mathrm{~h}$ and an MTT assay was performed using the Cell Proliferation Kit I (Roche) according to the manufacturer's protocol. (D) Expression levels of LEPR, cyclin D1, cyclin E, or beta-actin were examined by RT-PCR analyses. NT denotes control cell with no transfection. 
Table 1. Number of tumor tissues and non-tumorous cirrhotic liver tissues with somatic mutations at high (upper) and low (lower) frequencies in TP53, CTNNB1, and LEPR.

High-frequency mutations $(20 \%<)$

$$
\begin{gathered}
\text { Tumor }(\mathrm{n}=10) \\
\text { Non-tumor }(\mathrm{n}=22)
\end{gathered}
$$

Low-frequency mutations (1-20\%)

$$
\begin{gathered}
\text { Tumor }(n=10) \\
\text { Non-tumor }(n=22)
\end{gathered}
$$

0

$1^{*}$

9

12 *
1

0

8

17
1

0

*One patient had both high- and low-frequency mutations in LEPR. 
Table 2. Incidence of hepatic nodules in C57BL/KsJ- $d b / d b(d b / d b)$ and misty (control) mice after 24 or 30 weeks treatment with TAA.

\begin{tabular}{lcc}
\hline & $d b / d b$ & control \\
\hline \hline Male/Female & $(\mathrm{n}=10)$ & $(\mathrm{n}=10)$ \\
Tumor formation & $8 / 2$ & $8 / 2$ \\
$\quad$ Total & $4^{*}(40 \%)$ & $0(0 \%)$ \\
$\quad$ HCC & 2 & 0 \\
$\quad$ Hepatocyte hyperplasia & 3 & 0 \\
\hline \hline 30 weeks & $(\mathrm{n}=7)$ & $(\mathrm{n}=10)$ \\
\hline Male/Female & $3 / 4$ & $7 / 3$ \\
Tumor formation & & $4(40 \%)$ \\
Total & $6 *(86 \%)$ & 0 \\
$\quad$ HCC & 1 & 4 \\
$\quad$ Hepatocyte hyperplasia & 6 & \\
\hline
\end{tabular}

Numbers of animals that developed hepatocyte hyperplasia and/or hepatocellular carcinoma (HCC) are shown.

*One $d b / d b$ mouse developed both HCC and hepatocyte hyperplasia. 
Supplemental Table 1. Clinical features of 4 patients who underwent whole exome sequencing and 22 patients who underwent selected exome sequencing.

\begin{tabular}{|c|c|c|c|c|c|c|c|}
\hline Case & Age & Gender & BMIa & $\begin{array}{c}\text { AFPb } \\
(\mathrm{ng} / \mathrm{mL})\end{array}$ & $\begin{array}{c}\mathbf{D C P}^{\mathrm{c}} \\
(\mathrm{mAU} / \mathrm{mL})\end{array}$ & $\mathbf{M}^{\mathrm{d}}$ or $\mathrm{S}^{\mathrm{e}}$ & $\begin{array}{c}\text { Histological } \\
\text { grade }^{\mathrm{f}}\end{array}$ \\
\hline \multicolumn{8}{|c|}{ Whole exome sequencing } \\
\hline$\# 1$ & 51 & $\mathrm{M}$ & 23.3 & 16 & 185 & M & Wel \\
\hline$\# 2$ & 58 & $\mathrm{~F}$ & 22.3 & 103 & 7 & M & Mod \\
\hline$\# 3$ & 55 & $\mathrm{~F}$ & 26.7 & 779 & 881 & M & Mod \\
\hline$\# 4$ & 53 & $\mathrm{M}$ & 22.3 & 34 & 85 & $\mathrm{~S}$ & Mod \\
\hline \multicolumn{8}{|c|}{ Selected exome sequencing } \\
\hline$\# 5$ & 65 & M & 25.2 & 17 & & M & Mod \\
\hline$\# 6$ & 49 & $\mathrm{~F}$ & 21.6 & 149 & 07 & M & Mod \\
\hline \#7 & 40 & M & 25.7 & 24 & 50 & M & Mod \\
\hline$\# 8$ & 50 & M & 25.0 & 16 & 23 & M & Mod \\
\hline$\# 9$ & 57 & $\mathrm{~F}$ & 23.4 & 8 & 30 & $\mathrm{M}$ & Mod \\
\hline$\# 10$ & 56 & $\mathrm{~F}$ & 22.8 & 5 & 929 & M & Mod \\
\hline$\# 11$ & 53 & $\mathrm{M}$ & 18.6 & 30 & 31 & $\mathrm{M}$ & Mod \\
\hline$\# 12$ & 65 & $\mathrm{~F}$ & 29.7 & 6 & 1,877 & $\mathrm{~S}$ & Mod \\
\hline$\# 13$ & 57 & M & 19.0 & 19 & 167 & $\mathrm{~S}$ & Wel \\
\hline$\# 14$ & 76 & M & 21.8 & 75,363 & 37,784 & M & Por \\
\hline$\# 15$ & 64 & M & 18.7 & 177 & 8 & - & - \\
\hline$\# 16$ & 57 & M & 25.5 & 45 & 68 & - & - \\
\hline$\# 17$ & 54 & $\mathrm{~F}$ & 25.9 & $<3$ & 10 & - & - \\
\hline$\# 18$ & 50 & $\mathrm{M}$ & 22.3 & 585 & 61 & - & - \\
\hline$\# 19$ & 60 & $\mathrm{~F}$ & 21.3 & 434 & 72 & - & - \\
\hline$\# 20$ & 57 & M & 25.0 & 15 & 8,310 & - & - \\
\hline$\# 21$ & 56 & M & 19.0 & 15 & 383 & - & - \\
\hline$\# 22$ & 49 & $\mathrm{~F}$ & 21.8 & 38 & 227 & - & - \\
\hline$\# 23$ & 59 & $\mathrm{M}$ & 25.6 & 6 & 12 & - & - \\
\hline$\# 24$ & 49 & $\mathrm{M}$ & 23.2 & 4 & 320 & - & - \\
\hline$\# 25$ & 37 & $\mathrm{M}$ & 22.2 & 4 & 13 & - & - \\
\hline$\# 26$ & 51 & M & 20.5 & 3 & 90 & - & - \\
\hline
\end{tabular}

a: body mass index, b: $\alpha$-fetoprotein, c: des-Y-carboxy prothrombin, d: multiple HCCs, e: solitary HCC, f: Wel; well differentiated HCC, Mod; moderately differentiated HCC, Por; poorly differentiated HCC 
Supplemental Table 2. Overview of whole exome sequencing data of 4 HCC patients with HCV infection. Whole exome sequencing was performed on tumor tissues, non-tumorous cirrhotic liver tissues, and matched peripheral lymphocytes from each patient. Total reads, aligned reads, aligned sequences (bp), median read depth, and number of target regions, which were $1 \times, 8 \times, 20 \times$, and $30 \times$ or more coverage depth read, are shown.

\begin{tabular}{lccc}
\hline & Tumor $(\mathrm{n}=7)$ & Non-tumor $(\mathrm{n}=4)$ & Lymphocytes $(\mathrm{n}=\mathbf{4})$ \\
\hline Total reads & $44,323,036$ & $41,920,372$ & $38,661,394$ \\
Aligned reads & $40,046,800$ & $33,742,449$ & $31,595,571$ \\
Aligned sequence(bp) & $2,824,088,514$ & $2,384,058,470$ & $2,221,753,713$ \\
Median read depth & 40.2 & 31.9 & 27.4 \\
$\mathbf{1 \times} \quad$ Coverage & $31,560,125$ & $32,343,635$ & $30,935,484$ \\
$\mathbf{8} \times \quad$ Coverage & $24,724,702$ & $23,432,758$ & $23,549,909$ \\
$\mathbf{2 0}$ Coverage & $17,707,636$ & $15,000,474$ & $16,272,508$ \\
$\mathbf{3 0}$ Coverage & $13,599,418$ & $11,752,775$ & $12,527,511$ \\
\hline
\end{tabular}


Supplemental Table 3. List of 970 nucleotide positions in 768 genes that were mutated at a frequency of more than $20 \%$ of reads in 7 HCC tumors of 4 cases.

\begin{tabular}{|c|c|c|c|c|c|c|c|c|c|}
\hline Gene & $\begin{array}{c}\text { Reference } \\
\text { Position }\end{array}$ & $\mathrm{Chr}^{\mathrm{a}}$ & $\mathbf{C D S}^{\mathrm{b}}$ & Coverage & & $\begin{array}{l}\text { lele } \\
\text { inge }\end{array}$ & $\begin{array}{c}\text { Amino acid } \\
\text { change }^{c}\end{array}$ & Functio & Case \\
\hline AGRN & 875083 & 1 & 26 & 20 & A & $<\mathrm{C}$ & NS & D & $\# 2$ \\
\hline LOC728661 & 1487244 & 1 & 8 & 18 & G & $<\mathrm{T}$ & NS & $\mathrm{N}$ & $\# 3$ \\
\hline CDC2L2 & 1540787 & 1 & 3 & 43 & $\mathrm{~T}$ & $<\mathrm{C}$ & NS & $\mathrm{N}$ & $\# 4$ \\
\hline PANK4 & 2331358 & 1 & 18 & 32 & $\mathrm{~T}$ & $<\mathrm{C}$ & NS & $\mathrm{N}$ & $\# 4$ \\
\hline KIAA0562 & 3645675 & 1 & 7 & 67 & $\mathrm{~T}$ & $<\mathrm{C}$ & $\mathrm{S}$ & $\mathrm{N}$ & $\# 4$ \\
\hline CHD5 & 5928578 & 1 & 24 & 54 & $\mathrm{C}$ & $<\mathrm{T}$ & S & $\mathrm{N}$ & $\# 2$ \\
\hline PTCHD2 & 11319504 & 1 & 7 & 23 & $\mathrm{G}$ & $<\mathrm{C}$ & NS & $\mathrm{N}$ & $\# 4$ \\
\hline PLOD1 & 11750469 & 1 & 4 & 22 & $\mathrm{G}$ & & NS & $\mathrm{N}$ & $\# 4$ \\
\hline PRAMEF1 & 12595752 & 1 & 3 & 170 & G & $<\mathrm{A}$ & $\mathrm{S}$ & $\mathrm{N}$ & $\# 4$ \\
\hline PRAMEF1 & 12596087 & 1 & 3 & 93 & C & $<\mathrm{T}$ & NS & D & $\# 4$ \\
\hline PRAMEF11 & 12625168 & 1 & 5 & 48 & G & $<\mathrm{A}$ & $\mathrm{S}$ & $\mathrm{N}$ & $\# 4$ \\
\hline PRAMEF11 & 12628397 & 1 & 3 & 38 & C & $<\mathrm{T}$ & S & D & $\# 4$ \\
\hline PRAMEF11 & 12628415 & 1 & 3 & 36 & $\mathrm{C}$ & $<\mathrm{T}$ & $\mathrm{S}$ & $\mathrm{N}$ & $\# 4$ \\
\hline HNRNPCL1 & 12647885 & 1 & 1 & 143 & $\mathrm{~T}$ & $<\mathrm{C}$ & $\mathrm{S}$ & $\mathrm{N}$ & $\# 4$ \\
\hline PRAMEF7 & 12717626 & 1 & 1 & 27 & A & $<\mathrm{G}$ & S & $\mathrm{N}$ & $\# 4$ \\
\hline PRAMEF9* & 13064237 & 1 & & 26 & $\mathrm{G}$ & $<\mathrm{A}$ & NS & $\mathrm{N}$ & $\# 2$ \\
\hline PRAMEF9* & 13064255 & & 1 & 35 & $\mathrm{G}$ & $<\mathrm{A}$ & NS & $\mathrm{D}$ & $\# 2$ \\
\hline PRAMEF18 & 13117381 & 1 & 1 & 27 & $\mathrm{G}$ & $<\mathrm{A}$ & NS & $\mathrm{N}$ & $\# 4$ \\
\hline ARHGEF10L & 17547108 & 1 & 1 & 109 & $\mathrm{~T}$ & $<\mathrm{G}$ & $\mathrm{S}$ & $\mathrm{N}$ & $\# 4$ \\
\hline PLA2G2D & 20082054 & 1 & 3 & 56 & $\mathrm{~T}$ & $<\mathrm{C}$ & NS & $\mathrm{N}$ & $\# 4$ \\
\hline HSPG2 & 21856574 & 1 & 5 & 77 & $\mathrm{C}$ & $<\mathrm{A}$ & NS & $\mathrm{N}$ & $\# 4$ \\
\hline CELA3A & 21973988 & 1 & 6 & 105 & $\mathrm{~T}$ & $<\mathrm{G}$ & NS & $\mathrm{N}$ & $\# 4$ \\
\hline CELA3A & 21976308 & 1 & 7 & 49 & $\mathrm{G}$ & $<\mathrm{A}$ & $\mathrm{S}$ & $\mathrm{N}$ & $\# 4$ \\
\hline LOC 100289113 & 22086886 & 1 & 1 & 28 & $\mathrm{~A}$ & $<\mathrm{C}$ & NS & $\mathrm{D}$ & $\# 1$ \\
\hline LUZP1 & 23059855 & 1 & 1 & 48 & $\mathrm{~T}$ & $<\mathrm{C}$ & $\mathrm{S}$ & $\mathrm{N}$ & $\# 4$ \\
\hline TRIM63 & 26025003 & 1 & 5 & 90 & $\mathrm{~T}$ & $<\mathrm{C}$ & NS & $\mathrm{N}$ & $\# 4$ \\
\hline SLC9A1 & 27120757 & 1 & 1 & 56 & $\mathrm{~A}$ & $<\mathrm{G}$ & $\mathrm{S}$ & $\mathrm{N}$ & $\# 4$ \\
\hline $\mathrm{PHC} 2$ & 33310033 & 1 & 8 & 132 & $\mathrm{C}$ & $<\mathrm{T}$ & $\mathrm{s}$ & $\mathrm{N}$ & $\# 4$ \\
\hline CSMD2 & 33528214 & 1 & 51 & 83 & $\mathrm{~T}$ & $<\mathrm{C}$ & NS & $\mathrm{N}$ & $\# 4$ \\
\hline SLC2A1 & 42884612 & 1 & 8 & 74 & $\mathrm{~T}$ & $<\mathrm{C}$ & $\mathrm{S}$ & $\mathrm{N}$ & $\# 4$ \\
\hline
\end{tabular}




\begin{tabular}{|c|c|c|c|c|c|c|c|c|}
\hline TIE1 & 43269564 & 1 & 14 & 55 & $\mathrm{~T}$ & $<\mathrm{C}$ & $\mathrm{S}$ & $\mathrm{N}$ \\
\hline MAST2 & 45983460 & 1 & 17 & 45 & $\mathrm{~T}$ & $<\mathrm{G}$ & NS & $\mathrm{N}$ \\
\hline LRP8 & 53222315 & 1 & 9 & 143 & G & $<\mathrm{T}$ & $\mathrm{s}$ & $\mathrm{N}$ \\
\hline ANGPTL3 & 62554389 & 1 & 2 & 24 & A & $<\mathrm{T}$ & NS & $\mathrm{D}$ \\
\hline LEPR & 65548341 & 1 & 4 & 31 & $\mathrm{C}$ & $<\mathrm{A}$ & $\mathrm{s}$ & $\mathrm{N}$ \\
\hline RPE65 & 68386987 & 1 & 12 & 33 & A & $<\mathrm{C}$ & NS & $\mathrm{N}$ \\
\hline ZNF644 & 90894104 & 1 & 2 & 18 & G & $<\mathrm{A}$ & NS & $\mathrm{N}$ \\
\hline RBM15 & 110372981 & 1 & 1 & 17 & A & $<\mathrm{C}$ & $\mathrm{S}$ & $\mathrm{N}$ \\
\hline RBM15 & 110373546 & 1 & 1 & 39 & $\mathrm{~T}$ & $<\mathrm{C}$ & NS & $\mathrm{N}$ \\
\hline CHI3L2 & 111273982 & 1 & 9 & 79 & $\mathrm{C}$ & $<\mathrm{T}$ & NS & $\mathrm{N}$ \\
\hline CSDE1 & 114765324 & 1 & 8 & 29 & $\mathrm{C}$ & $<\mathrm{A}$ & NS & $\mathrm{N}$ \\
\hline CSDE1 & 114765325 & 1 & 8 & 29 & $\mathrm{C}$ & $<\mathrm{A}$ & NS & $\mathrm{N}$ \\
\hline IGSF3 & 116648924 & 1 & 2 & 69 & G & $<$ & S & NO \\
\hline NBPF20 & 122618548 & 1 & 15 & 62 & G & $<\mathrm{A}$ & $\mathrm{S}$ & $\mathrm{N}$ \\
\hline NBPF20 & 122618618 & 1 & 15 & 140 & C & $<\mathrm{T}$ & NS & $\mathrm{N}$ \\
\hline NBPF20 & 122618624 & 1 & 15 & 174 & A & $<\mathrm{T}$ & NS & $\mathrm{N}$ \\
\hline PDE4DIP & 122663887 & 1 & 31 & 88 & C & $<\mathrm{T}$ & $\mathrm{S}$ & $\mathrm{N}$ \\
\hline PDE4DIP & 122667176 & 1 & 28 & 71 & $\mathrm{C}$ & $<\mathrm{T}$ & NS & $\mathrm{N}$ \\
\hline NBPF10 & 123083515 & 1 & 1 & 83 & A & $<\mathrm{G}$ & NS & $\mathrm{N}$ \\
\hline NBPF10 & 123092695 & 1 & 8 & 17 & $\mathrm{C}$ & $<\mathrm{T}$ & NS & $\mathrm{N}$ \\
\hline NBPF10 & 123094578 & 1 & 10 & 100 & A & $<\mathrm{C}$ & NS & $\mathrm{N}$ \\
\hline NBPF10 & 123094595 & 1 & 10 & 217 & A & $<\mathrm{G}$ & NS & $\mathrm{N}$ \\
\hline NBPF10 & 123158473 & 1 & 86 & 408 & G & $<\mathrm{C}$ & NS & $\mathrm{N}$ \\
\hline ANKRD35 & 123351469 & 1 & 10 & 14 & A & $<\mathrm{T}$ & NS & $\mathrm{N}$ \\
\hline GPR89C & 123673973 & 1 & 1 & 23 & $\mathrm{~T}$ & $<\mathrm{G}$ & NS & $\mathrm{D}$ \\
\hline BCL9 & 124884100 & 1 & 6 & 18 & G & $<\mathrm{A}$ & $\mathrm{S}$ & $\mathrm{N}$ \\
\hline NBPF14 & 125797806 & 1 & 18 & 56 & $\mathrm{C}$ & $<\mathrm{T}$ & NS & $\mathrm{N}$ \\
\hline NBPF14 & 125799375 & 1 & 16 & 76 & $\mathrm{~T}$ & $<\mathrm{C}$ & $\mathrm{S}$ & $\mathrm{N}$ \\
\hline NBPF14 & 125799402 & 1 & 16 & 71 & G & $<\mathrm{A}$ & $\mathrm{S}$ & $\mathrm{N}$ \\
\hline NBPF15 & 126071852 & 1 & 4 & 159 & A & $<\mathrm{G}$ & $\mathrm{S}$ & $\mathrm{N}$ \\
\hline HRNR & 129676605 & 1 & 2 & 214 & G & $<\mathrm{A}$ & s & $\mathrm{N}$ \\
\hline HRNR & 129676984 & 1 & 2 & 52 & G & $<\mathrm{C}$ & NS & NO \\
\hline HRNR & 129677003 & 1 & 2 & 88 & $\mathrm{C}$ & $<\mathrm{T}$ & NS & $\mathrm{N}$ \\
\hline $\mathrm{FLG}^{*}$ & 129766583 & 1 & 2 & 89 & $\mathrm{C}$ & $<\mathrm{G}$ & NS & $\mathrm{N}$ \\
\hline FLG & 129767962 & 1 & 2 & 102 & $\mathrm{C}$ & $<\mathrm{T}$ & NS & $\mathrm{N}$ \\
\hline FLG & 129768306 & 1 & 2 & 39 & $\mathrm{~T}$ & $<\mathrm{C}$ & NS & $\mathrm{N}$ \\
\hline
\end{tabular}




\begin{tabular}{|c|c|c|c|c|c|c|c|c|c|}
\hline FLG & 129768312 & 1 & 2 & 59 & $\mathrm{C}$ & $<\mathrm{G}$ & NS & $\mathrm{N}$ & \#4 \\
\hline FLG & 129771039 & 1 & 2 & 449 & G & $<\mathrm{A}$ & NS & $\mathrm{N}$ & $\# 2$ \\
\hline FLG & 129771228 & 1 & 2 & 298 & $\mathrm{C}$ & $<\mathrm{G}$ & NS & $\mathrm{N}$ & \#4 \\
\hline FLG & 129773236 & 1 & 2 & 135 & $\mathrm{~T}$ & $<\mathrm{C}$ & NS & $\mathrm{N}$ & $\# 2$ \\
\hline FLG & 129773862 & 1 & 2 & 222 & G & $<\mathrm{C}$ & NS & $\mathrm{N}$ & \#4 \\
\hline FLG & 129774814 & 1 & 2 & 232 & $\mathrm{~T}$ & $<\mathrm{C}$ & NS & $\mathrm{N}$ & \#4 \\
\hline PGLYRP3 & 130769598 & 1 & 2 & 118 & $\mathrm{C}$ & $<\mathrm{T}$ & $\mathrm{S}$ & $\mathrm{N}$ & $\# 4$ \\
\hline CLK2 & 132724561 & 1 & 8 & 17 & $\mathrm{C}$ & $<\mathrm{T}$ & NS & D & $\# 3$ \\
\hline CLK2 & 132724562 & 1 & 8 & 17 & G & $<\mathrm{T}$ & $\mathrm{S}$ & $\mathrm{N}$ & \#3 \\
\hline MSTO1 & 133072971 & 1 & 11 & 33 & $\mathrm{~T}$ & $<\mathrm{G}$ & $\mathrm{S}$ & $\mathrm{N}$ & $\# 3$ \\
\hline GON4L & 133214185 & 1 & 27 & 50 & $\mathrm{C}$ & $<\mathrm{A}$ & NS & $\mathrm{D}$ & $\# 2$ \\
\hline IQGAP3 & 134016387 & 1 & 12 & 66 & $\mathrm{C}$ & $<\mathrm{G}$ & NS & $\mathrm{N}$ & $\# 4$ \\
\hline PEA15 & 137673244 & 1 & 3 & 25 & A & & NS & $\mathrm{N}$ & \#3 \\
\hline HSPA6 & 138985040 & 1 & 1 & 34 & $\mathrm{C}$ & $<$ & NS & $\mathrm{D}$ & \#4 \\
\hline NUF2 & 140800188 & 1 & 8 & 38 & $\mathrm{C}$ & $<\mathrm{A}$ & NS & NO & \#3 \\
\hline FAM78B & 143529898 & 1 & 2 & 204 & C & $<\mathrm{G}$ & $\mathrm{S}$ & $\mathrm{N}$ & $\# 4$ \\
\hline F5 & 147009112 & 1 & 10 & 162 & C & $<\mathrm{T}$ & NS & $\mathrm{N}$ & \#4 \\
\hline FAM5C* & 167558142 & 1 & 7 & 33 & G & $<\mathrm{A}$ & NS & $\mathrm{N}$ & \#1 \\
\hline ZBTB41 & 174618823 & 1 & 10 & 13 & A & $<\mathrm{C}$ & NS & NO & $\# 2$ \\
\hline KIF21B* & 178450152 & 1 & 18 & 56 & $\mathrm{~T}$ & $<\mathrm{C}$ & $\mathrm{S}$ & $\mathrm{N}$ & \#1 \\
\hline TMEM9 & 178602981 & 1 & & 127 & A & $<\mathrm{G}$ & $\mathrm{s}$ & $\mathrm{N}$ & \#4 \\
\hline ELF3 & 179471218 & 1 & 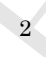 & 54 & $\mathrm{C}$ & $<\mathrm{G}$ & $\mathrm{S}$ & $\mathrm{N}$ & \#4 \\
\hline PPP1R12B & 180023641 & 1 & 21 & 22 & $\mathrm{C}$ & $<\mathrm{A}$ & NS & $\mathrm{N}$ & $\# 3$ \\
\hline KDM5B & 180267325 & 1 & 1 & 13 & $\mathrm{G}$ & $<\mathrm{C}$ & NS & $\mathrm{D}$ & $\# 1$ \\
\hline CHI3L1 & 180642801 & 1 & 5 & 141 & $\mathrm{~T}$ & $<\mathrm{C}$ & NS & $\mathrm{D}$ & \#4 \\
\hline FAM71A & 189989294 & 1 & 1 & 133 & $\mathrm{~T}$ & $<\mathrm{C}$ & NS & $\mathrm{N}$ & \#3 \\
\hline MIA3 & 200015587 & 1 & 13 & 40 & $\mathrm{~T}$ & $<\mathrm{C}$ & $\mathrm{S}$ & $\mathrm{N}$ & \#4 \\
\hline JMJD4 & 205110357 & 1 & 6 & 53 & C & $<\mathrm{T}$ & $\mathrm{s}$ & $\mathrm{N}$ & \#4 \\
\hline OBSCN* & 205602418 & 1 & 8 & 22 & $\mathrm{~T}$ & $<\mathrm{C}$ & NS & $\mathrm{D}$ & $\# 1$ \\
\hline RHOU & 206063445 & 1 & 2 & 52 & C & $<\mathrm{G}$ & $\mathrm{S}$ & $\mathrm{N}$ & \#4 \\
\hline GNPAT & 208576822 & 1 & 2 & 47 & $\mathrm{C}$ & $<\mathrm{T}$ & NS & $\mathrm{D}$ & \#3 \\
\hline LYST & 213162183 & 1 & 3 & 14 & G & $<\mathrm{T}$ & NS & $\mathrm{N}$ & \#2 \\
\hline ADSS & 221776216 & 1 & 7 & 22 & A & $<\mathrm{C}$ & NS & $\mathrm{D}$ & \#3 \\
\hline ADSS & 221776218 & 1 & 7 & 22 & C & $<\mathrm{T}$ & $\mathrm{S}$ & $\mathrm{N}$ & $\# 3$ \\
\hline KIF26B & 223037622 & 1 & 11 & 110 & $\mathrm{C}$ & $<\mathrm{T}$ & $\mathrm{S}$ & $\mathrm{N}$ & \#4 \\
\hline LOC391343 & 227830117 & 2 & 1 & 41 & $\mathrm{~T}$ & $<\mathrm{G}$ & NS & NR & $\# 4$ \\
\hline
\end{tabular}




\begin{tabular}{|c|c|c|c|c|c|c|c|c|c|}
\hline LOC391343 & 227830313 & 2 & 1 & 16 & $\mathrm{G}$ & $<\mathrm{C}$ & $\mathrm{S}$ & NR & $\# 3$ \\
\hline PXDN & 228577682 & 2 & 17 & 144 & G & $<\mathrm{C}$ & NS & $\mathrm{N}$ & \#4 \\
\hline ODC1 & 237355517 & 2 & 10 & 27 & $\mathrm{G}$ & $<\mathrm{A}$ & $\mathrm{S}$ & $\mathrm{N}$ & \#4 \\
\hline АРОВ & 247947498 & 2 & 16 & 24 & A & $<\mathrm{C}$ & NS & $\mathrm{N}$ & \#3 \\
\hline $\mathrm{APOB}$ & 247947499 & 2 & 16 & 24 & A & $<\mathrm{T}$ & NS & $\mathrm{N}$ & \#3 \\
\hline ALK & 256154817 & 2 & 15 & 59 & A & $<\mathrm{G}$ & $\mathrm{S}$ & $\mathrm{N}$ & $\# 4$ \\
\hline FSHR & 275890251 & 2 & 10 & 21 & G & $<\mathrm{T}$ & NS & NO & \#3 \\
\hline C2orf63 & 282104344 & 2 & 10 & 39 & G & $<\mathrm{A}$ & NS & $\mathrm{N}$ & \#4 \\
\hline CYP26B1 & 299058969 & 2 & 6 & 23 & $\mathrm{G}$ & $<\mathrm{A}$ & $\mathrm{S}$ & $\mathrm{N}$ & $\# 1$ \\
\hline CCDC142 & 301407728 & 2 & 4 & 37 & G & $<\mathrm{A}$ & NS & NO & $\# 2$ \\
\hline ST3GAL5 & 312787861 & 2 & 3 & 17 & $\mathrm{~T}$ & $<\mathrm{C}$ & NS & $\mathrm{N}$ & $\# 4$ \\
\hline KIAA1310 & 319723935 & 2 & 13 & 14 & G & $<\mathrm{T}$ & NS & $\mathrm{N}$ & $\# 3$ \\
\hline ACTR1B & 320724551 & 2 & 6 & 276 & C & & $\mathrm{S}$ & $\mathrm{N}$ & $\# 2$ \\
\hline CHST10 & 323459632 & 2 & 5 & 57 & $\mathrm{G}$ & $<$ & $\mathrm{S}$ & $\mathrm{N}$ & \#4 \\
\hline MAP4K4 & 324943015 & 2 & 26 & 37 & G & $<\mathrm{C}$ & NS & $\mathrm{D}$ & $\# 3$ \\
\hline SLC9A4 & 325569668 & 2 & 3 & 69 & A & $<\mathrm{T}$ & NS & $\mathrm{D}$ & \#3 \\
\hline TGFBRAP1 & 328335511 & 2 & 10 & 51 & $\mathrm{~T}$ & $<\mathrm{C}$ & NS & $\mathrm{N}$ & \#4 \\
\hline RGPD3 & 329534289 & 2 & 1 & 37 & A & $<\mathrm{G}$ & $\mathrm{s}$ & $\mathrm{N}$ & $\# 4$ \\
\hline LIMS1 & 331725649 & 2 & 1 & 96 & G & $<\mathrm{A}$ & NS & $\mathrm{D}$ & $\# 4$ \\
\hline 10-Sep & 332632987 & 2 & 6 & 104 & G & $<\mathrm{A}$ & $\mathrm{S}$ & $\mathrm{N}$ & $\# 4$ \\
\hline LOC645529 & 336733665 & 2 & & 118 & $\mathrm{~T}$ & $<\mathrm{C}$ & NS & NR & \#4 \\
\hline POTEF & 353150970 & 2 & & 98 & $\mathrm{~T}$ & $<\mathrm{A}$ & NS & $\mathrm{N}$ & \#4 \\
\hline POTEF & 353185302 & 2 & 1 & 71 & $\mathrm{~T}$ & $<\mathrm{C}$ & NS & $\mathrm{N}$ & \#4 \\
\hline TUBA3E & 353260275 & 2 & 3 & 94 & $\mathrm{C}$ & $<\mathrm{T}$ & NS & $\mathrm{N}$ & $\# 2$ \\
\hline ACTBL3 & 354657198 & 2 & 1 & 108 & $\mathrm{~T}$ & $<\mathrm{G}$ & $\mathrm{S}$ & NR & \#4 \\
\hline THSD7B & 360341133 & 2 & 11 & 14 & $\mathrm{G}$ & $<\mathrm{C}$ & $\mathrm{S}$ & $\mathrm{N}$ & $\# 2$ \\
\hline GALNT5 & 380364795 & 2 & 7 & 19 & $\mathrm{~T}$ & $<\mathrm{G}$ & NS & $\mathrm{D}$ & \#3 \\
\hline SCN9A & 389348565 & 2 & 11 & 38 & $\mathrm{~T}$ & $<\mathrm{C}$ & NS & $\mathrm{N}$ & \#3 \\
\hline SCN9A & 389352545 & 2 & 9 & 39 & $\mathrm{~T}$ & $<\mathrm{C}$ & $\mathrm{S}$ & $\mathrm{N}$ & $\# 4$ \\
\hline ABCB11 & 391996481 & 2 & 23 & 27 & $\mathrm{G}$ & $<\mathrm{A}$ & NS & NO & \#3 \\
\hline OLA1 & 397151333 & 2 & 9 & 14 & A & $<\mathrm{T}$ & $\mathrm{S}$ & $\mathrm{N}$ & \#3 \\
\hline TTN & 401781960 & 2 & 95 & 24 & $\mathrm{~T}$ & $<\mathrm{G}$ & NS & $\mathrm{D}$ & $\# 3$ \\
\hline TTN & 401781961 & 2 & 95 & 24 & $\mathrm{C}$ & $<\mathrm{T}$ & $\mathrm{S}$ & $\mathrm{N}$ & $\# 3$ \\
\hline SESTD1 & 402189020 & 2 & 14 & 28 & $\mathrm{G}$ & $<\mathrm{T}$ & NS & $\mathrm{D}$ & $\# 3$ \\
\hline DUSP19 & 406151282 & 2 & 1 & 41 & $\mathrm{C}$ & $<\mathrm{G}$ & NS & $\mathrm{D}$ & \#3 \\
\hline ZNF804A & 408011183 & 2 & 4 & 20 & A & $<\mathrm{G}$ & $\mathrm{S}$ & $\mathrm{N}$ & \#3 \\
\hline
\end{tabular}




\begin{tabular}{|c|c|c|c|c|c|c|c|c|c|}
\hline LOC200726 & 429716908 & 2 & 1 & 28 & $\mathrm{C}$ & $<\mathrm{T}$ & NS & NR & \#3 \\
\hline ERBB4 & 434456096 & 2 & 28 & 29 & $\mathrm{C}$ & $<\mathrm{T}$ & NS & $\mathrm{N}$ & \#3 \\
\hline RNF25 & 441737431 & 2 & 8 & 18 & G & $<\mathrm{A}$ & $\mathrm{S}$ & $\mathrm{N}$ & $\# 3$ \\
\hline C2orf24 & 442244994 & 2 & 8 & 51 & G & $<\mathrm{A}$ & NS & $\mathrm{N}$ & $\# 4$ \\
\hline C2orf24 & 442245306 & 2 & 8 & 31 & A & $<\mathrm{G}$ & NS & $\mathrm{N}$ & \#4 \\
\hline TUBA4A & 442323330 & 2 & 4 & 103 & $\mathrm{C}$ & $<\mathrm{T}$ & NS & D & \#4 \\
\hline OBSL1 & 442639564 & 2 & 4 & 59 & G & $<\mathrm{A}$ & $\mathrm{S}$ & $\mathrm{N}$ & $\# 4$ \\
\hline SERPINE2 & 447057057 & 2 & 5 & 24 & $\mathrm{G}$ & $<\mathrm{A}$ & $\mathrm{S}$ & $\mathrm{N}$ & $\# 3$ \\
\hline DOCK10 & 447917888 & 2 & 20 & 54 & G & $<\mathrm{A}$ & NS & NO & \#1 \\
\hline DIS3L2 & 455310852 & 2 & 10 & 90 & $\mathrm{G}$ & $<\mathrm{A}$ & NS & $\mathrm{N}$ & \#1 \\
\hline ALPP & 455451136 & 2 & 1 & 40 & $\mathrm{C}$ & $<\mathrm{T}$ & NS & D & \#4 \\
\hline LRRFIP1 & 460828821 & 2 & 11 & 16 & A & $<\mathrm{G}$ & $\mathrm{S}$ & $\mathrm{N}$ & \#1 \\
\hline HDAC4 & 462131420 & 2 & 20 & 77 & G & & $\mathrm{S}$ & $\mathrm{N}$ & \#4 \\
\hline ITPR1 & 469948734 & 3 & 21 & 68 & A & & $\mathrm{s}$ & $\mathrm{N}$ & \#4 \\
\hline WNT7A & 479128207 & 3 & 3 & 47 & $\mathrm{C}$ & $<\mathrm{T}$ & $\mathrm{S}$ & $\mathrm{N}$ & \#4 \\
\hline ZFYVE20 & 480358270 & 3 & 5 & 13 & C & $<\mathrm{T}$ & NS & $\mathrm{N}$ & $\# 3$ \\
\hline OXNAD1 & 481544487 & 3 & 1 & 101 & C & $<\mathrm{T}$ & $\mathrm{S}$ & $\mathrm{N}$ & $\# 4$ \\
\hline RARB & 490854079 & 3 & 5 & 14 & $\mathrm{~T}$ & $<\mathrm{C}$ & $\mathrm{S}$ & $\mathrm{N}$ & \#3 \\
\hline EOMES & 492992244 & 3 & 4 & 84 & $\mathrm{C}$ & $<\mathrm{T}$ & NS & $\mathrm{N}$ & $\# 1$ \\
\hline SCN10A & 504030094 & 3 & 9 & 56 & $\mathrm{C}$ & $<\mathrm{T}$ & $\mathrm{S}$ & $\mathrm{N}$ & $\# 4$ \\
\hline SCN11A* & 504168069 & 3 & 15 & 110 & $\mathrm{~T}$ & $<\mathrm{A}$ & NS & NO & $\# 1$ \\
\hline CX3CR1 & 504539250 & 3 & & 36 & $\mathrm{~T}$ & $<\mathrm{C}$ & NS & $\mathrm{N}$ & $\# 3$ \\
\hline CTNNB1 & 506498027 & 3 & 2 & 39 & G & $<\mathrm{A}$ & NS & $\mathrm{D}$ & $\# 1$ \\
\hline CCR5 & 511646366 & 3 & 1 & 20 & $\mathrm{C}$ & $<\mathrm{A}$ & NS & NO & $\# 3$ \\
\hline COL7A1 & 513857189 & 3 & 21 & 50 & $\mathrm{~T}$ & $<\mathrm{C}$ & $\mathrm{S}$ & $\mathrm{N}$ & $\# 4$ \\
\hline RBM6 & 515335643 & 3 & 16 & 16 & $\mathrm{G}$ & $<\mathrm{A}$ & NS & D & $\# 3$ \\
\hline RBM5 & 515386440 & 3 & 22 & 46 & $\mathrm{G}$ & $<\mathrm{A}$ & NS & $\mathrm{D}$ & $\# 3$ \\
\hline GLYCTK & 517558462 & 3 & 4 & 64 & $\mathrm{C}$ & $<\mathrm{A}$ & $\mathrm{S}$ & $\mathrm{N}$ & $\# 2$ \\
\hline KBTBD8 & 532186608 & 3 & 2 & 48 & G & $<\mathrm{T}$ & NS & NO & \#3 \\
\hline FOXP1 & 536153715 & 3 & 13 & 189 & A & $<\mathrm{C}$ & NS & D & $\# 3$ \\
\hline FOXP1 & 536153718 & 3 & 13 & 190 & C & $<\mathrm{T}$ & $\mathrm{S}$ & $\mathrm{N}$ & $\# 3$ \\
\hline LOC100288801 & 540845478 & 3 & 1 & 220 & $\mathrm{G}$ & $<\mathrm{A}$ & NS & $\mathrm{N}$ & $\# 4$ \\
\hline LOC100288801* & 540846776 & 3 & 2 & 228 & G & $<\mathrm{A}$ & $\mathrm{S}$ & $\mathrm{N}$ & $\# 2$ \\
\hline ЕРНА3 & 554308286 & 3 & 2 & 14 & $\mathrm{~T}$ & $<\mathrm{C}$ & $\mathrm{S}$ & $\mathrm{N}$ & $\# 3$ \\
\hline DCBLD2 & 560650518 & 3 & 16 & 37 & G & $<\mathrm{A}$ & NS & $\mathrm{N}$ & $\# 3$ \\
\hline DCBLD2 & 560650520 & 3 & 16 & 35 & A & $<\mathrm{C}$ & NS & NO & \#3 \\
\hline
\end{tabular}




\begin{tabular}{|c|c|c|c|c|c|c|c|c|c|}
\hline $\mathrm{BOC}$ & 575123882 & 3 & 6 & 56 & $\mathrm{C}$ & $<\mathrm{T}$ & S & $\mathrm{N}$ & $\# 4$ \\
\hline GPR156 & 582017947 & 3 & 9 & 64 & A & $<\mathrm{T}$ & S & $\mathrm{N}$ & $\# 2$ \\
\hline HEG1 & 586864008 & 3 & 6 & 41 & $\mathrm{~T}$ & $<\mathrm{C}$ & NS & $\mathrm{N}$ & $\# 3$ \\
\hline MCM2* & 589457039 & 3 & 5 & 53 & A & $<\mathrm{G}$ & NS & $\mathrm{N}$ & \#1 \\
\hline RUVBL1 & 589951418 & 3 & 6 & 33 & $\mathrm{~T}$ & $<\mathrm{A}$ & NS & $\mathrm{D}$ & $\# 3$ \\
\hline RUVBL1 & 589951420 & 3 & 6 & 30 & $\mathrm{~T}$ & $<\mathrm{G}$ & NS & D & $\# 3$ \\
\hline C3orf25 & 591272422 & 3 & 2 & 44 & $\mathrm{~T}$ & $<\mathrm{C}$ & NS & D & \#4 \\
\hline PLXND1 & 591434995 & 3 & 7 & 116 & G & $<\mathrm{A}$ & $\mathrm{S}$ & $\mathrm{N}$ & $\# 4$ \\
\hline COL6A6 & 592486478 & 3 & 27 & 23 & G & $<\mathrm{T}$ & NS & $\mathrm{D}$ & $\# 3$ \\
\hline SLCO2A1 & 595793430 & 3 & 11 & 60 & A & $<\mathrm{G}$ & NS & $\mathrm{D}$ & $\# 1$ \\
\hline RYK & 596026424 & 3 & 13 & 102 & $\mathrm{C}$ & $<\mathrm{T}$ & NS & $\mathrm{N}$ & $\# 1$ \\
\hline ZBTB38* & 603296515 & 3 & 1 & 39 & A & $<\mathrm{G}$ & NS & $\mathrm{N}$ & $\# 1$ \\
\hline PLOD2 & 607970905 & 3 & 3 & 11 & $\mathrm{~T}$ & $<$ & $\mathrm{S}$ & $\mathrm{N}$ & $\# 2$ \\
\hline PLSCR2 & 608303872 & 3 & 4 & 55 & A & $<\mathrm{T}$ & NS & $\mathrm{D}$ & $\# 3$ \\
\hline TMEM183B & 611832538 & 3 & 1 & 44 & A & $<\mathrm{C}$ & NS & NR & $\# 3$ \\
\hline TSC22D2 & 612260917 & 3 & 1 & 14 & $\mathrm{~T}$ & $<\mathrm{G}$ & S & $\mathrm{N}$ & $\# 4$ \\
\hline MYNN & 631624024 & 3 & 1 & 54 & C & $<\mathrm{T}$ & $\mathrm{S}$ & $\mathrm{N}$ & $\# 4$ \\
\hline TNIK* & 633027074 & 3 & 8 & 63 & $\mathrm{C}$ & $<\mathrm{G}$ & NS & $\mathrm{D}$ & $\# 1$ \\
\hline IL1RAP & 652454036 & 3 & 2 & 73 & $\mathrm{C}$ & $<\mathrm{A}$ & $\mathrm{S}$ & $\mathrm{N}$ & $\# 2$ \\
\hline MUC4 & 657634114 & 3 & 3 & 76 & A & $<\mathrm{G}$ & NS & $\mathrm{N}$ & $\# 4$ \\
\hline MUC4 & 657640844 & 3 & 2 & 214 & $\mathrm{~T}$ & $<\mathrm{C}$ & NS & $\mathrm{N}$ & $\# 4$ \\
\hline FGFRL1 & 661098114 & 4 & 6 & 16 & $\mathrm{C}$ & $<\mathrm{A}$ & NS & $\mathrm{N}$ & $\# 3$ \\
\hline TNIP2 & 662767650 & 4 & 6 & 33 & G & $<\mathrm{A}$ & NS & $\mathrm{N}$ & $\# 4$ \\
\hline LOC100288212 & 680710684 & 4 & 2 & 44 & G & $<\mathrm{A}$ & S & $\mathrm{N}$ & $\# 3$ \\
\hline GPR125 & 682521774 & 4 & 1 & 21 & C & $<\mathrm{A}$ & NS & $\mathrm{N}$ & $\# 2$ \\
\hline TBC1D1 & 698120919 & 4 & 19 & 29 & G & $<\mathrm{A}$ & NS & $\mathrm{N}$ & \#4 \\
\hline SCFD2 & 711062849 & 4 & 1 & 22 & A & $<\mathrm{C}$ & NS & $\mathrm{D}$ & $\# 3$ \\
\hline SCFD2 & 711062850 & 4 & 1 & 24 & G & $<\mathrm{T}$ & NS & $\mathrm{N}$ & $\# 3$ \\
\hline KIAA1211 & 714010832 & 4 & 4 & 25 & A & $<\mathrm{G}$ & $\mathrm{NS}$ & $\mathrm{D}$ & \#3 \\
\hline UGT2B28 & 726937878 & 4 & 5 & 40 & A & $<\mathrm{G}$ & $\mathrm{s}$ & $\mathrm{N}$ & $\# 3$ \\
\hline UGT2B28 & 726937879 & 4 & 5 & 39 & A & $<\mathrm{T}$ & NS & $\mathrm{D}$ & $\# 3$ \\
\hline SULT1B1 & 727380561 & 4 & 5 & 28 & A & $<\mathrm{C}$ & NS & $\mathrm{D}$ & $\# 3$ \\
\hline ENAM & 728278770 & 4 & 2 & 31 & G & $<\mathrm{T}$ & NS & $\mathrm{N}$ & \#3 \\
\hline ANKRD17 & 730738780 & 4 & 29 & 56 & $\mathrm{~T}$ & $<\mathrm{A}$ & $\mathrm{s}$ & $\mathrm{N}$ & \#3 \\
\hline ANKRD17 & 730738782 & 4 & 29 & 58 & A & $<\mathrm{G}$ & NS & $\mathrm{N}$ & $\# 3$ \\
\hline FRAS1 & 735932986 & 4 & 6 & 28 & $\mathrm{~T}$ & $<\mathrm{A}$ & NS & NO & \#3 \\
\hline
\end{tabular}




\begin{tabular}{|c|c|c|c|c|c|c|c|c|c|}
\hline FRAS1 & 735932989 & 4 & 6 & 28 & $\mathrm{C}$ & $<\mathrm{T}$ & $\mathrm{S}$ & $\mathrm{N}$ & \#3 \\
\hline AFF1 & 744724579 & 4 & 3 & 25 & G & $<\mathrm{A}$ & $\mathrm{S}$ & $\mathrm{N}$ & \#3 \\
\hline SPARCL1 & 745172651 & 4 & 2 & 66 & G & $<\mathrm{T}$ & NS & $\mathrm{N}$ & $\# 4$ \\
\hline HERC6 & 746068457 & 4 & 4 & 52 & $\mathrm{~T}$ & $<\mathrm{A}$ & NS & $\mathrm{N}$ & $\# 4$ \\
\hline CXXC4 & 762168791 & 4 & 1 & 23 & $\mathrm{C}$ & $<\mathrm{T}$ & NS & $\mathrm{N}$ & $\# 3$ \\
\hline PDE5A & 777231322 & 4 & 8 & 81 & A & $<\mathrm{G}$ & $\mathrm{S}$ & $\mathrm{N}$ & $\# 4$ \\
\hline FAT4 & 783168335 & 4 & 17 & 26 & A & $<\mathrm{T}$ & NS & D & $\# 3$ \\
\hline FAT4 & 783168337 & 4 & 17 & 25 & G & $<\mathrm{T}$ & NS & $\mathrm{N}$ & $\# 3$ \\
\hline INPP4B* & 799948368 & 4 & 7 & 144 & $\mathrm{C}$ & $<\mathrm{A}$ & NS & $\mathrm{N}$ & $\# 1$ \\
\hline EDNRA & 805163608 & 4 & 1 & 58 & $\mathrm{C}$ & $<\mathrm{T}$ & $\mathrm{S}$ & $\mathrm{N}$ & \#3 \\
\hline RBM $46^{*}$ & 812505537 & 4 & 4 & 102 & $\mathrm{~T}$ & $<\mathrm{C}$ & NS & $\mathrm{N}$ & $\# 1$ \\
\hline ACCN5 & 813543803 & 4 & 1 & 41 & G & $<\mathrm{A}$ & $\mathrm{S}$ & $\mathrm{N}$ & $\# 4$ \\
\hline 1-Mar & 821206546 & 4 & 4 & 89 & $\mathrm{C}$ & $<$ & $\mathrm{S}$ & $\mathrm{N}$ & $\# 4$ \\
\hline DDX60 & 825930266 & 4 & 26 & 15 & $\mathrm{C}$ & $<\mathrm{T}$ & NS & $\mathrm{N}$ & \#3 \\
\hline DDX60 & 825930267 & 4 & 26 & 15 & A & $<\mathrm{T}$ & NS & $\mathrm{N}$ & \#3 \\
\hline MFAP3L & 827669812 & 4 & 2 & 33 & G & $<\mathrm{C}$ & NS & $\mathrm{D}$ & \#3 \\
\hline AGA & 835115128 & 4 & 5 & 41 & $\mathrm{~T}$ & $<\mathrm{A}$ & $\mathrm{S}$ & $\mathrm{N}$ & $\# 3$ \\
\hline IRF2 & 842095828 & 4 & 4 & 19 & $\mathrm{~T}$ & $<\mathrm{C}$ & NS & $\mathrm{D}$ & \#3 \\
\hline SORBS2* & 843292510 & 4 & 13 & 232 & $\mathrm{C}$ & $<\mathrm{T}$ & NS & $\mathrm{D}$ & $\# 1$ \\
\hline FAM149A & 843833669 & 4 & 4 & 50 & A & $<\mathrm{G}$ & NS & $\mathrm{D}$ & $\# 4$ \\
\hline FAT1 & 844287485 & 4 & 14 & 146 & A & $<\mathrm{G}$ & NS & $\mathrm{N}$ & $\# 4$ \\
\hline FAT1 & 844298124 & 4 & 9 & 19 & G & $<\mathrm{A}$ & NS & $\mathrm{D}$ & \#3 \\
\hline TRIML2 & 845769469 & 4 & 7 & 40 & $\mathrm{C}$ & $<\mathrm{T}$ & NS & $\mathrm{N}$ & $\# 2$ \\
\hline FRG2 & 847704735 & 4 & 1 & 72 & $\mathrm{~T}$ & $<\mathrm{A}$ & NS & $\mathrm{N}$ & $\# 4$ \\
\hline MAFIP & 848044252 & 4 & 7 & 56 & C & $<\mathrm{G}$ & $\mathrm{S}$ & NR & $\# 4$ \\
\hline MAFIP & 848046049 & 4 & 4 & 33 & G & $<\mathrm{A}$ & $\mathrm{S}$ & NR & $\# 4$ \\
\hline SLC6A18 & 849416422 & 5 & 10 & 180 & $\mathrm{C}$ & $<\mathrm{T}$ & NS & $\mathrm{N}$ & $\# 4$ \\
\hline NDUFS6 & 849988000 & 5 & 4 & 70 & G & $<\mathrm{T}$ & $\mathrm{S}$ & $\mathrm{N}$ & $\# 3$ \\
\hline TRIO & 862540906 & 5 & 17 & 113 & $\mathrm{C}$ & $<\mathrm{T}$ & $\mathrm{S}$ & $\mathrm{N}$ & $\# 2$ \\
\hline ANKH & 862913981 & 5 & 8 & 116 & $\mathrm{~T}$ & $<\mathrm{C}$ & $\mathrm{S}$ & $\mathrm{N}$ & $\# 4$ \\
\hline FBXL7 & 864100358 & 5 & 3 & 48 & A & $<\mathrm{G}$ & NS & $\mathrm{N}$ & $\# 2$ \\
\hline RNASEN & 879698242 & 5 & 2 & 141 & G & $<\mathrm{A}$ & $\mathrm{S}$ & $\mathrm{N}$ & $\# 4$ \\
\hline ADAMTS12 & 881707057 & 5 & 23 & 45 & G & $<\mathrm{A}$ & NS & $\mathrm{N}$ & $\# 4$ \\
\hline EGFLAM & 886579974 & 5 & 9 & 30 & $\mathrm{~T}$ & $<\mathrm{G}$ & NS & NO & $\# 3$ \\
\hline EGFLAM & 886610442 & 5 & 17 & 63 & G & $<\mathrm{A}$ & $\mathrm{S}$ & $\mathrm{N}$ & $\# 2$ \\
\hline CD180 & 911650805 & 5 & 3 & 38 & G & $<\mathrm{A}$ & $\mathrm{S}$ & $\mathrm{N}$ & $\# 3$ \\
\hline
\end{tabular}




\begin{tabular}{|c|c|c|c|c|c|c|c|c|c|}
\hline MARVELD2 & 913887307 & 5 & 1 & 47 & $\mathrm{C}$ & $<\mathrm{T}$ & NS & $\mathrm{N}$ & $\# 4$ \\
\hline WDR41 & 921906081 & 5 & 10 & 129 & $\mathrm{C}$ & $<\mathrm{T}$ & NS & $\mathrm{N}$ & \#4 \\
\hline GDF9 & 977321994 & 5 & 1 & 37 & $\mathrm{G}$ & $<\mathrm{T}$ & NS & $\mathrm{D}$ & \#3 \\
\hline C5orf15 & 978417392 & 5 & 2 & 18 & $\mathrm{G}$ & $<\mathrm{A}$ & $\mathrm{S}$ & $\mathrm{N}$ & $\# 3$ \\
\hline KIF20A & 982639408 & 5 & 3 & 29 & $\mathrm{~T}$ & $<\mathrm{C}$ & $\mathrm{S}$ & $\mathrm{N}$ & \#3 \\
\hline КDM3В & 982876692 & 5 & 14 & 17 & $\mathrm{~T}$ & $<\mathrm{C}$ & $\mathrm{S}$ & $\mathrm{N}$ & \#4 \\
\hline LOC202051 & 983854576 & 5 & 6 & 61 & C & $<\mathrm{T}$ & $\mathrm{S}$ & $\mathrm{N}$ & \#4 \\
\hline PCDHB11 & 985652769 & 5 & 1 & 24 & $\mathrm{~T}$ & $<\mathrm{C}$ & $\mathrm{S}$ & $\mathrm{N}$ & \#4 \\
\hline HMHB1 & 988272050 & 5 & 2 & 125 & $\mathrm{C}$ & $<\mathrm{T}$ & NS & $\mathrm{D}$ & \#4 \\
\hline ABLIM3* & 993692261 & 5 & 13 & 55 & A & $<\mathrm{G}$ & $\mathrm{S}$ & $\mathrm{N}$ & $\# 1$ \\
\hline PDGFRB & 994581527 & 5 & 9 & 24 & $\mathrm{~A}$ & $<\mathrm{C}$ & NS & $\mathrm{D}$ & $\# 1$ \\
\hline NDST1 & 994979638 & 5 & 2 & 57 & $\mathrm{C}$ & $<\mathrm{T}$ & $\mathrm{S}$ & $\mathrm{N}$ & \#4 \\
\hline NDST1 & 994984515 & 5 & 3 & 14 & $\mathrm{C}$ & $<$ & NS & $\mathrm{N}$ & \#3 \\
\hline KIF4B & 999468839 & 5 & 1 & 50 & $\mathrm{C}$ & $<\mathrm{A}$ & $\mathrm{S}$ & $\mathrm{N}$ & \#3 \\
\hline KIF4B & 999468844 & 5 & 1 & 48 & A & $<\mathrm{C}$ & NS & $\mathrm{N}$ & \#3 \\
\hline ADAM19 & 1001937293 & 5 & 21 & 26 & C & $<\mathrm{A}$ & NS & $\mathrm{N}$ & \#3 \\
\hline FBXW11* & 1016325320 & 5 & 8 & 98 & $\mathrm{~T}$ & $<\mathrm{C}$ & NS & $\mathrm{D}$ & $\# 1$ \\
\hline C5orf47 & 1018438284 & 5 & 1 & 27 & $\mathrm{~T}$ & $<\mathrm{G}$ & $\mathrm{S}$ & $\mathrm{N}$ & \#3 \\
\hline FGFR4 & 1021542240 & 5 & 8 & 26 & $\mathrm{G}$ & $<\mathrm{A}$ & NS & $\mathrm{N}$ & \#4 \\
\hline FLT4 & 1025068341 & 5 & 19 & 33 & G & $<\mathrm{C}$ & NS & $\mathrm{D}$ & \#4 \\
\hline BTNL3 & 1025454701 & 5 & & 38 & $\mathrm{G}$ & $<\mathrm{T}$ & $\mathrm{S}$ & $\mathrm{N}$ & \#3 \\
\hline TUBB2A & 1029022083 & 6 & 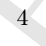 & 28 & A & $<\mathrm{G}$ & $\mathrm{S}$ & $\mathrm{N}$ & \#3 \\
\hline LRRC16A & 1051287607 & 6 & 3 & 142 & A & $<\mathrm{C}$ & $\mathrm{S}$ & $\mathrm{N}$ & \#4 \\
\hline SLC17A4 & 1051637752 & 6 & 3 & 191 & $\mathrm{C}$ & $<\mathrm{T}$ & $\mathrm{S}$ & $\mathrm{N}$ & \#4 \\
\hline BTN3A2 & 1052237964 & 6 & 3 & 68 & $\mathrm{~T}$ & $<\mathrm{C}$ & $\mathrm{S}$ & $\mathrm{N}$ & \#4 \\
\hline HLA-G & 1055664896 & 6 & 5 & 85 & $\mathrm{C}$ & $<\mathrm{T}$ & $\mathrm{s}$ & $\mathrm{N}$ & $\# 4$ \\
\hline HLA-A & 1055777815 & 6 & 2 & 33 & $\mathrm{~T}$ & $<\mathrm{A}$ & NS & $\mathrm{N}$ & $\# 2$ \\
\hline HLA-A* & 1055777819 & 6 & 2 & 85 & $\mathrm{~A}$ & $<\mathrm{C}$ & $\mathrm{S}$ & $\mathrm{N}$ & $\# 2$ \\
\hline $\mathrm{C} 4 \mathrm{~A}$ & 1057829599 & 6 & 21 & 20 & $\mathrm{~T}$ & $<\mathrm{G}$ & NS & $\mathrm{D}$ & $\# 2$ \\
\hline TNXB & 1057902726 & 6 & 17 & 35 & G & $<\mathrm{A}$ & $\mathrm{S}$ & $\mathrm{N}$ & \#4 \\
\hline BTNL2* & 1058229998 & 6 & 6 & 218 & $\mathrm{C}$ & $<\mathrm{T}$ & NS & $\mathrm{N}$ & $\# 2$ \\
\hline BTNL2* & 1058230002 & 6 & 6 & 220 & $\mathrm{G}$ & $<\mathrm{A}$ & NS & $\mathrm{N}$ & $\# 2$ \\
\hline HLA-DRB1 & 1058415838 & 6 & 4 & 83 & A & $<\mathrm{G}$ & $\mathrm{S}$ & $\mathrm{N}$ & $\# 4$ \\
\hline HLA-DQB1 & 1058497104 & 6 & 3 & 50 & $\mathrm{~A}$ & $<\mathrm{G}$ & $\mathrm{S}$ & $\mathrm{N}$ & \#2 \\
\hline HLA-DPB1 & 1058920866 & 6 & 4 & 94 & $\mathrm{G}$ & $<\mathrm{A}$ & NS & $\mathrm{N}$ & $\# 4$ \\
\hline GRM4 & 1059927081 & 6 & 2 & 94 & $\mathrm{C}$ & $<\mathrm{T}$ & NS & $\mathrm{D}$ & $\# 2$ \\
\hline
\end{tabular}




\begin{tabular}{|c|c|c|c|c|c|c|c|c|c|}
\hline C6orf127 & 1061622915 & 6 & 3 & 78 & A & $<\mathrm{G}$ & $\mathrm{S}$ & $\mathrm{N}$ & \#4 \\
\hline SRPK1 & 1061704552 & 6 & 11 & 58 & $\mathrm{G}$ & $<\mathrm{T}$ & $\mathrm{S}$ & $\mathrm{N}$ & $\# 3$ \\
\hline SLC26A8 & 1061790503 & 6 & 16 & 180 & $\mathrm{~T}$ & $<\mathrm{C}$ & NS & $\mathrm{N}$ & $\# 4$ \\
\hline TREML2 & 1067029775 & 6 & 3 & 241 & $\mathrm{~T}$ & $<\mathrm{C}$ & NS & $\mathrm{N}$ & $\# 4$ \\
\hline TTBK1 & 1069098172 & 6 & 12 & 37 & $\mathrm{G}$ & $<\mathrm{C}$ & NS & $\mathrm{N}$ & $\# 2$ \\
\hline HSP90AB1 & 1070084817 & 6 & 3 & 24 & $\mathrm{G}$ & $<\mathrm{A}$ & NS & $\mathrm{N}$ & $\# 2$ \\
\hline GPR116 & 1072716510 & 6 & 7 & 52 & $\mathrm{~A}$ & $<\mathrm{C}$ & $\mathrm{S}$ & $\mathrm{N}$ & $\# 3$ \\
\hline CD2AP & 1073430855 & 6 & 12 & 58 & $\mathrm{~T}$ & $<\mathrm{A}$ & NS & $\mathrm{N}$ & $\# 3$ \\
\hline PKHD1 & 1077359171 & 6 & 65 & 80 & $\mathrm{~T}$ & $<\mathrm{A}$ & $\mathrm{NS}$ & $\mathrm{N}$ & \#3 \\
\hline GSTA2 & 1078484988 & 6 & 4 & 89 & $\mathrm{C}$ & $<\mathrm{G}$ & $\mathrm{NS}$ & $\mathrm{N}$ & \#4 \\
\hline GFRAL & 1081063844 & 6 & 2 & 32 & $\mathrm{C}$ & $<\mathrm{T}$ & NS & $\mathrm{N}$ & \#4 \\
\hline PRIM2 & 1083334432 & 6 & 10 & 62 & A & $<\mathrm{G}$ & $\mathrm{S}$ & NR & \#4 \\
\hline PRIM2 & 1083379733 & 6 & 13 & 50 & $\mathrm{G}$ & & NS & NR & \#4 \\
\hline PRIM2 & 1083379822 & 6 & 13 & 79 & $\mathrm{~T}$ & $<$ & NS & NR & \#4 \\
\hline EYS & 1087198904 & 6 & 40 & 25 & $\mathrm{G}$ & $<\mathrm{T}$ & $\mathrm{S}$ & $\mathrm{N}$ & \#3 \\
\hline EYS & 1087198906 & 6 & 40 & 19 & G & $<\mathrm{C}$ & NS & $\mathrm{N}$ & $\# 3$ \\
\hline IMPG1 & 1099519071 & 6 & 2 & 37 & $\mathrm{~T}$ & $<\mathrm{C}$ & NS & $\mathrm{N}$ & \#3 \\
\hline ME1 & 1106705882 & 6 & 10 & 19 & $\mathrm{~T}$ & $<\mathrm{A}$ & $\mathrm{NS}$ & $\mathrm{D}$ & \#3 \\
\hline ME1 & 1106705883 & 6 & 10 & 17 & $\mathrm{~T}$ & $<\mathrm{A}$ & NS & NO & \#3 \\
\hline GABRR1 & 1112675095 & 6 & 5 & 172 & $\mathrm{~A}$ & $<\mathrm{G}$ & NS & $\mathrm{N}$ & $\# 2$ \\
\hline MDN1 & 1113138459 & 6 & 88 & 90 & $\mathrm{G}$ & $<\mathrm{A}$ & $\mathrm{S}$ & $\mathrm{N}$ & \#4 \\
\hline WISP3 & 1134999625 & 6 & & 157 & $\mathrm{~T}$ & $<\mathrm{G}$ & NS & $\mathrm{D}$ & \#4 \\
\hline HS3ST5 & 1136996498 & 6 & 2 & 16 & $\mathrm{C}$ & $<\mathrm{T}$ & NS & $\mathrm{N}$ & \#3 \\
\hline COL10A1 & 1139059549 & 6 & 2 & 97 & A & $<\mathrm{G}$ & $\mathrm{S}$ & $\mathrm{N}$ & \#2 \\
\hline NKAIN2* & 1147293746 & 6 & 3 & 247 & $\mathrm{C}$ & $<\mathrm{G}$ & NS & NO & $\# 1$ \\
\hline PERP* & 1161034772 & 6 & 2 & 61 & $\mathrm{~T}$ & $<\mathrm{C}$ & NS & $\mathrm{N}$ & \#1 \\
\hline HEBP2 & 1161351286 & 6 & 4 & 56 & $\mathrm{~A}$ & $<\mathrm{C}$ & $\mathrm{S}$ & $\mathrm{N}$ & \#4 \\
\hline NHSL1 & 1161385538 & 6 & 4 & 32 & $\mathrm{G}$ & $<\mathrm{T}$ & NS & $\mathrm{D}$ & \#3 \\
\hline HIVEP2 & 1165711211 & 6 & 1 & 34 & $\mathrm{C}$ & $<\mathrm{T}$ & NS & $\mathrm{D}$ & $\# 3$ \\
\hline SAMD5 & 1170447330 & 6 & 1 & 31 & $\mathrm{C}$ & $<\mathrm{T}$ & $\mathrm{S}$ & $\mathrm{N}$ & \#4 \\
\hline PCMT1 & 1172688307 & 6 & 1 & 41 & $\mathrm{~T}$ & $<\mathrm{C}$ & NS & $\mathrm{D}$ & \#3 \\
\hline ZBTB2 & 1174303968 & 6 & 2 & 18 & $\mathrm{~T}$ & $<\mathrm{A}$ & NS & $\mathrm{N}$ & $\# 3$ \\
\hline SYNE1 & 1175082156 & 6 & 136 & 30 & $\mathrm{C}$ & $<\mathrm{T}$ & $\mathrm{S}$ & $\mathrm{N}$ & \#3 \\
\hline SYNJ2 & 1181103116 & 6 & 11 & 37 & $\mathrm{~T}$ & $<\mathrm{G}$ & NS & $\mathrm{N}$ & $\# 3$ \\
\hline TULP4 & 1181540258 & 6 & 13 & 24 & $\mathrm{~A}$ & $<\mathrm{C}$ & NS & $\mathrm{N}$ & \#3 \\
\hline RSPH3 & 1182019083 & 6 & 6 & 43 & A & $<\mathrm{T}$ & NS & $\mathrm{D}$ & \#3 \\
\hline
\end{tabular}




\begin{tabular}{|c|c|c|c|c|c|c|c|c|c|}
\hline IGF2R & 1183085535 & 6 & 16 & 66 & $\mathrm{~A}$ & $<\mathrm{G}$ & $\mathrm{S}$ & $\mathrm{N}$ & $\# 4$ \\
\hline AGPAT4 & 1184192475 & 6 & 3 & 85 & $\mathrm{C}$ & $<\mathrm{T}$ & NS & $\mathrm{N}$ & $\# 2$ \\
\hline MLLT4 & 1190935073 & 6 & 19 & 75 & A & $<\mathrm{C}$ & $\mathrm{S}$ & $\mathrm{N}$ & $\# 4$ \\
\hline FAM120B & 1193244908 & 6 & 1 & 53 & G & $<\mathrm{A}$ & $\mathrm{S}$ & $\mathrm{N}$ & $\# 4$ \\
\hline ADAP1 & 1194606192 & 7 & 6 & 69 & G & $<\mathrm{A}$ & $\mathrm{S}$ & $\mathrm{N}$ & $\# 4$ \\
\hline MICALL2 & 1195144333 & 7 & 7 & 58 & G & $<\mathrm{C}$ & $\mathrm{S}$ & & $\# 4$ \\
\hline SDK1 & 1197713015 & 7 & 15 & 37 & G & $<\mathrm{T}$ & NS & $\mathrm{D}$ & \#3 \\
\hline RSPH10B & 1199630320 & 7 & 18 & 54 & $\mathrm{C}$ & $<\mathrm{G}$ & $\mathrm{S}$ & $\mathrm{N}$ & $\# 3$ \\
\hline VWDE & 1206072045 & 7 & 12 & 19 & G & $<\mathrm{T}$ & NS & $\mathrm{N}$ & \#3 \\
\hline HDAC $9^{*}$ & 1212495378 & 7 & 16 & 176 & A & $<\mathrm{T}$ & NS & $\mathrm{D}$ & $\# 1$ \\
\hline TMEM196 & 1213427541 & 7 & 3 & 117 & G & $<\mathrm{T}$ & $\mathrm{S}$ & $\mathrm{N}$ & $\# 2$ \\
\hline ITGB8* & 1214065640 & 7 & 2 & 100 & G & $<\mathrm{C}$ & NS & $\mathrm{D}$ & $\# 1$ \\
\hline C7orf10 & 1234451360 & 7 & 14 & 36 & $\mathrm{~T}$ & $<$ & NS & $\mathrm{N}$ & $\# 3$ \\
\hline C7orf10 & 1234451361 & 7 & 14 & 38 & $\mathrm{~T}$ & $<\mathrm{G}$ & NS & $\mathrm{NO}$ & \#3 \\
\hline AEBP1 & 1237814514 & 7 & 18 & 22 & A & $<\mathrm{T}$ & NS & $\mathrm{N}$ & $\# 1$ \\
\hline MYO1G & 1238671649 & 7 & 11 & 87 & G & $<\mathrm{A}$ & $\mathrm{S}$ & $\mathrm{N}$ & $\# 4$ \\
\hline C7orf65 & 1241361009 & 7 & 3 & 60 & A & $<\mathrm{G}$ & $\mathrm{S}$ & $\mathrm{N}$ & $\# 4$ \\
\hline ABCA13 & 1242074221 & 7 & 33 & 18 & A & $<\mathrm{C}$ & NS & $\mathrm{D}$ & $\# 2$ \\
\hline ABCA13 & 1242105751 & 7 & 39 & 33 & $\mathrm{C}$ & $<\mathrm{T}$ & $\mathrm{s}$ & $\mathrm{N}$ & $\# 3$ \\
\hline LOC100289307 & 1263515207 & 7 & 2 & 24 & G & $<\mathrm{T}$ & NS & NR & $\# 2$ \\
\hline MLXIPL & 1263542408 & 7 & & 13 & $\mathrm{C}$ & $<\mathrm{T}$ & NS & $\mathrm{D}$ & $\# 1$ \\
\hline SPDYE5 & 1265649637 & 7 & 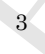 & 130 & G & $<\mathrm{A}$ & $\mathrm{s}$ & NR & \#4 \\
\hline POR & 1266124069 & 7 & 2 & 11 & G & $<\mathrm{T}$ & $\mathrm{s}$ & $\mathrm{N}$ & \#3 \\
\hline $\mathrm{HGF}$ & 1271854360 & 7 & 18 & 27 & $\mathrm{~T}$ & $<\mathrm{A}$ & NS & $\mathrm{D}$ & $\# 3$ \\
\hline SEMA3E & 1273551762 & 7 & 11 & 83 & C & $<\mathrm{T}$ & $\mathrm{S}$ & $\mathrm{N}$ & $\# 4$ \\
\hline SEMA3A & 1274113176 & 7 & 17 & 291 & $\mathrm{~T}$ & $<\mathrm{C}$ & $\mathrm{S}$ & $\mathrm{N}$ & \#4 \\
\hline FZD1 & 1281417809 & 7 & 1 & 72 & G & $<\mathrm{T}$ & $\mathrm{S}$ & $\mathrm{N}$ & $\# 3$ \\
\hline SAMD9 & 1283257403 & 7 & 1 & 18 & $\mathrm{~T}$ & $<\mathrm{A}$ & NS & $\mathrm{N}$ & $\# 1$ \\
\hline PVRIG & 1290339880 & 7 & 1 & 17 & $\mathrm{C}$ & $<\mathrm{T}$ & NS & $\mathrm{N}$ & $\# 1$ \\
\hline MUC12 & 1291135269 & 7 & 1 & 14 & $\mathrm{C}$ & $<\mathrm{T}$ & $\mathrm{S}$ & $\mathrm{N}$ & $\# 1$ \\
\hline MUC12 & 1291159789 & 7 & 5 & 42 & C & $<\mathrm{T}$ & $\mathrm{S}$ & $\mathrm{N}$ & $\# 4$ \\
\hline MUC12 & 1291160135 & 7 & 5 & 46 & G & $<\mathrm{A}$ & NS & $\mathrm{N}$ & $\# 4$ \\
\hline MUC12 & 1291160603 & 7 & 5 & 15 & $\mathrm{~T}$ & $<\mathrm{C}$ & NS & $\mathrm{N}$ & $\# 4$ \\
\hline MUC12 & 1291161436 & 7 & 5 & 14 & G & $<\mathrm{C}$ & $\mathrm{S}$ & $\mathrm{N}$ & $\# 4$ \\
\hline MUC12 & 1291161644 & 7 & 5 & 52 & $\mathrm{C}$ & $<\mathrm{T}$ & NS & $\mathrm{N}$ & $\# 4$ \\
\hline MUC12 & 1291165668 & 7 & 5 & 25 & G & $<\mathrm{T}$ & NS & $\mathrm{D}$ & $\# 4$ \\
\hline
\end{tabular}




\begin{tabular}{|c|c|c|c|c|c|c|c|c|c|}
\hline MUC12 & 1291165672 & 7 & 5 & 29 & A & $<\mathrm{C}$ & $\mathrm{S}$ & $\mathrm{N}$ & $\# 4$ \\
\hline MUC12 & 1291166156 & 7 & 5 & 79 & $\mathrm{C}$ & $<\mathrm{G}$ & NS & $\mathrm{N}$ & \#4 \\
\hline MUC12 & 1291166192 & 7 & 5 & 26 & $\mathrm{C}$ & $<\mathrm{A}$ & NS & $\mathrm{N}$ & $\# 4$ \\
\hline MUC17 & 1291200140 & 7 & 3 & 165 & G & $<\mathrm{A}$ & NS & $\mathrm{N}$ & $\# 4$ \\
\hline MUC17 & 1291201640 & 7 & 3 & 120 & $\mathrm{~T}$ & $<\mathrm{A}$ & NS & $\mathrm{N}$ & $\# 4$ \\
\hline MUC17 & 1291203834 & 7 & 3 & 37 & $\mathrm{~T}$ & $<\mathrm{C}$ & $\mathrm{S}$ & $\mathrm{N}$ & $\# 2$ \\
\hline PLOD3 & 1291376235 & 7 & 13 & 27 & G & $<\mathrm{C}$ & NS & D & $\# 4$ \\
\hline LOC100132214 & 1292658130 & 7 & 12 & 31 & $\mathrm{~A}$ & $<\mathrm{G}$ & NS & $\mathrm{D}$ & $\# 4$ \\
\hline IFRD1 & 1302624679 & 7 & 8 & 44 & $\mathrm{~T}$ & $<\mathrm{G}$ & $\mathrm{S}$ & $\mathrm{N}$ & $\# 4$ \\
\hline POT1 & 1314997694 & 7 & 11 & 13 & $\mathrm{C}$ & $<\mathrm{T}$ & NS & $\mathrm{N}$ & $\# 2$ \\
\hline POT1 & 1314997698 & 7 & 11 & 12 & A & $<\mathrm{G}$ & $\mathrm{s}$ & $\mathrm{N}$ & $\# 2$ \\
\hline CALD1 & 1325140404 & 7 & 3 & 19 & A & $<\mathrm{T}$ & NS & $\mathrm{N}$ & $\# 3$ \\
\hline TMEM140 & 1325371901 & 7 & 1 & 64 & A & $<c$ & $\mathrm{~S}$ & $\mathrm{~N}$ & $\# 4$ \\
\hline JHDM1D & 1330288269 & 7 & 20 & 22 & A & $<\mathrm{T}$ & $\mathrm{S}$ & $\mathrm{N}$ & $\# 3$ \\
\hline JHDM1D & 1330288270 & 7 & 20 & 24 & A & $<\mathrm{T}$ & NS & $\mathrm{D}$ & $\# 3$ \\
\hline TRBV7-7 & 1332617173 & 7 & 2 & 155 & $\mathrm{~T}$ & $<\mathrm{C}$ & $\mathrm{S}$ & NR & $\# 4$ \\
\hline TRBV20-1 & 1332996157 & 7 & 5 & 110 & A & $<\mathrm{T}$ & NS & NR & $\# 4$ \\
\hline TRBV20-1 & 1332996161 & 7 & 5 & 114 & $\mathrm{C}$ & $<\mathrm{T}$ & NS & NR & $\# 4$ \\
\hline LOC441294 & 1333766303 & 7 & 1 & 41 & G & $<\mathrm{A}$ & $\mathrm{S}$ & NR & \#3 \\
\hline LOC441294 & 1333766306 & 7 & 1 & 41 & A & $<\mathrm{T}$ & $\mathrm{S}$ & NR & $\# 3$ \\
\hline CTAGE4 & 1334380174 & 7 & & 29 & A & $<\mathrm{T}$ & NS & $\mathrm{N}$ & $\# 3$ \\
\hline ARHGEF5L & 1334381935 & 7 & & 16 & $\mathrm{~T}$ & $<\mathrm{G}$ & NS & $\mathrm{D}$ & $\# 2$ \\
\hline EZH2 & 1339023228 & 7 & 5 & 106 & $\mathrm{C}$ & $<\mathrm{G}$ & NS & $\mathrm{N}$ & $\# 4$ \\
\hline AGAP3 & 1341312932 & 7 & 7 & 15 & G & $<\mathrm{T}$ & $\mathrm{S}$ & $\mathrm{N}$ & $\# 1$ \\
\hline MLL3 & 1342375605 & 7 & 36 & 28 & G & $<\mathrm{A}$ & NS & $\mathrm{D}$ & $\# 3$ \\
\hline DLGAP2 & 1351315601 & 8 & 5 & 24 & A & $<\mathrm{G}$ & $\mathrm{s}$ & $\mathrm{N}$ & $\# 4$ \\
\hline MCPH1 & 1356177925 & 8 & 13 & 40 & $\mathrm{C}$ & $<\mathrm{T}$ & $\mathrm{S}$ & $\mathrm{N}$ & $\# 4$ \\
\hline FAM90A15 & 1356815370 & 8 & 4 & 30 & $\mathrm{C}$ & $<\mathrm{G}$ & NS & NR & \#2 \\
\hline TNKS & 1359086641 & 8 & 2 & 19 & A & $<\mathrm{T}$ & NS & $\mathrm{D}$ & $\# 2$ \\
\hline TNKS & 1359086644 & 8 & 2 & 17 & G & $<\mathrm{C}$ & NS & $\mathrm{D}$ & $\# 2$ \\
\hline RP1L1 & 1360114631 & 8 & 3 & 25 & $\mathrm{~T}$ & $<\mathrm{C}$ & NS & $\mathrm{N}$ & \#4 \\
\hline RP1L1 & 1360116520 & 8 & 3 & 23 & $\mathrm{~T}$ & $<\mathrm{C}$ & NS & $\mathrm{N}$ & $\# 2$ \\
\hline C8orf74 & 1360204184 & 8 & 3 & 75 & G & $<\mathrm{T}$ & NS & $\mathrm{D}$ & $\# 4$ \\
\hline MTUS1 & 1367102384 & 8 & 14 & 33 & G & $<\mathrm{A}$ & S & $\mathrm{N}$ & $\# 4$ \\
\hline DOCK5 & 1374758768 & 8 & 10 & 113 & G & $<\mathrm{A}$ & $\mathrm{S}$ & $\mathrm{N}$ & $\# 4$ \\
\hline C8orf41 & 1382968877 & 8 & 1 & 13 & A & $<\mathrm{G}$ & $\mathrm{S}$ & $\mathrm{N}$ & $\$ 4$ \\
\hline
\end{tabular}




\begin{tabular}{|c|c|c|c|c|c|c|c|c|c|}
\hline CHRNA6 & 1392210295 & 8 & 5 & 36 & G & $<\mathrm{T}$ & $\mathrm{S}$ & $\mathrm{N}$ & \#1 \\
\hline KCNB2 & 1420442160 & 8 & 2 & 17 & $\mathrm{~A}$ & $<\mathrm{C}$ & NS & $\mathrm{D}$ & $\# 3$ \\
\hline JPH1 & 1421750684 & 8 & 4 & 41 & $\mathrm{~T}$ & $<\mathrm{C}$ & NS & $\mathrm{N}$ & $\# 3$ \\
\hline ZFHX4 & 1424211672 & 8 & 1 & 17 & $\mathrm{C}$ & $<\mathrm{T}$ & $\mathrm{S}$ & $\mathrm{N}$ & $\# 3$ \\
\hline $\mathrm{CA} 2$ & 1432983132 & 8 & 6 & 24 & $\mathrm{G}$ & $<\mathrm{A}$ & NS & $\mathrm{D}$ & $\# 3$ \\
\hline REXO1L1 & 1433167938 & 8 & 1 & 21 & $\mathrm{G}$ & $<\mathrm{C}$ & NS & D & $\# 4$ \\
\hline LOC 100289448 & 1433170685 & 8 & 1 & 17 & $\mathrm{G}$ & $<\mathrm{C}$ & NS & NR & $\# 4$ \\
\hline RNF19A & 1447714815 & 8 & 9 & 14 & $\mathrm{C}$ & $<\mathrm{A}$ & NS & $\mathrm{N}$ & $\# 2$ \\
\hline ANGPT1 & 1454778129 & 8 & 4 & 54 & G & $<\mathrm{T}$ & NS & $\mathrm{N}$ & $\# 3$ \\
\hline ANGPT1 & 1454778131 & 8 & 4 & 45 & $\mathrm{~T}$ & $<\mathrm{C}$ & NS & $\mathrm{D}$ & $\# 3$ \\
\hline COL14A1 & 1467659774 & 8 & 8 & 87 & $\mathrm{~T}$ & $<\mathrm{C}$ & S & $\mathrm{N}$ & $\# 4$ \\
\hline ZHX1 & 1470709988 & 8 & 1 & 42 & $\mathrm{G}$ & $<\mathrm{T}$ & NS & $\mathrm{N}$ & $\# 3$ \\
\hline $\mathrm{TG}$ & 1480325843 & 8 & 3 & 113 & $\mathrm{G}$ & & NS & $\mathrm{N}$ & $\# 2$ \\
\hline COL22A1 & 1486144992 & 8 & 36 & 63 & $\mathrm{G}$ & $<$ & NS & $\mathrm{N}$ & $\# 4$ \\
\hline FLJ43860 & 1488920394 & 8 & 19 & 104 & G & $<\mathrm{A}$ & $\mathrm{S}$ & NR & $\# 4$ \\
\hline CYP11B2 & 1490439544 & 8 & 5 & 202 & C & $<\mathrm{T}$ & $\mathrm{S}$ & $\mathrm{N}$ & $\# 4$ \\
\hline LY6H & 1490684040 & 8 & 3 & 122 & C & $<\mathrm{G}$ & S & $\mathrm{N}$ & $\# 4$ \\
\hline KIAA0020 & 1495552364 & 9 & 1 & 35 & $\mathrm{C}$ & $<\mathrm{T}$ & NS & $\mathrm{N}$ & $\# 4$ \\
\hline CNTLN & 1510054815 & 9 & 11 & 13 & A & $<\mathrm{T}$ & NS & NO & $\# 3$ \\
\hline LINGO2 & 1520664425 & 9 & 1 & 19 & G & $<\mathrm{T}$ & $\mathrm{S}$ & $\mathrm{N}$ & \#3 \\
\hline PRSS3 & 1526511755 & 9 & & 163 & $\mathrm{~A}$ & $<\mathrm{G}$ & NS & $\mathrm{N}$ & $\# 4$ \\
\hline PRSS3 & 1526512490 & 9 & & 108 & $\mathrm{~T}$ & $<\mathrm{C}$ & $\mathrm{S}$ & $\mathrm{N}$ & \#4 \\
\hline $\mathrm{VCP}$ & 1527776116 & 9 & 8 & 30 & $\mathrm{~T}$ & $<\mathrm{C}$ & $\mathrm{NS}$ & $\mathrm{D}$ & \#3 \\
\hline FAM75A1 & 1532072293 & 9 & 4 & 27 & $\mathrm{C}$ & $<\mathrm{T}$ & NS & $\mathrm{N}$ & \#2 \\
\hline ALDH1A1* & 1548388439 & 9 & 11 & 85 & $\mathrm{G}$ & $<\mathrm{A}$ & NS & $\mathrm{D}$ & $\# 1$ \\
\hline TLE1 & 1557099343 & 9 & 9 & 23 & $\mathrm{G}$ & $<\mathrm{T}$ & NS & $\mathrm{N}$ & $\# 3$ \\
\hline TLE1 & 1557099344 & 9 & 9 & 21 & $\mathrm{~T}$ & $<\mathrm{C}$ & NS & $\mathrm{N}$ & $\# 3$ \\
\hline WNK2 & 1568934596 & 9 & 27 & 77 & A & $<\mathrm{G}$ & NS & $\mathrm{D}$ & $\# 3$ \\
\hline C9orf129 & 1568961550 & 9 & 2 & 47 & $\mathrm{~T}$ & $<\mathrm{C}$ & NS & $\mathrm{N}$ & $\# 3$ \\
\hline PTCH1* & 1571085902 & 9 & 17 & 111 & $\mathrm{~T}$ & $<\mathrm{C}$ & NS & $\mathrm{N}$ & $\# 1$ \\
\hline GRIN3A & 1577199512 & 9 & 9 & 20 & $\mathrm{~A}$ & $<\mathrm{T}$ & NS & $\mathrm{N}$ & $\# 3$ \\
\hline MUSK & 1586295091 & 9 & 1 & 57 & $\mathrm{G}$ & $<\mathrm{A}$ & NS & $\mathrm{N}$ & \#3 \\
\hline FKBP15 & 1588814600 & 9 & 13 & 33 & $\mathrm{~A}$ & $<\mathrm{C}$ & NS & $\mathrm{N}$ & $\# 4$ \\
\hline COL27A1 & 1589933932 & 9 & 59 & 118 & A & $<\mathrm{G}$ & NS & $\mathrm{N}$ & $\# 4$ \\
\hline ORM2 & 1589957833 & 9 & 4 & 37 & $\mathrm{G}$ & $<\mathrm{C}$ & NS & D & $\# 4$ \\
\hline GSN & 1596958694 & 9 & 17 & 159 & $\mathrm{~T}$ & $<\mathrm{C}$ & $\mathrm{S}$ & $\mathrm{N}$ & \#4 \\
\hline
\end{tabular}




\begin{tabular}{|c|c|c|c|c|c|c|c|c|c|}
\hline MAPKAP1 & 1601110716 & 9 & 8 & 14 & A & $<\mathrm{T}$ & $\mathrm{S}$ & $\mathrm{N}$ & \#3 \\
\hline CDK9 & 1603414267 & 9 & 4 & 23 & A & $<\mathrm{G}$ & NS & $\mathrm{D}$ & $\# 3$ \\
\hline C9orf78 & 1605455403 & 9 & 8 & 32 & A & $<\mathrm{G}$ & $\mathrm{S}$ & $\mathrm{N}$ & \#4 \\
\hline C9orf98 & 1608412608 & 9 & 9 & 23 & $\mathrm{~T}$ & $<\mathrm{A}$ & NS & $\mathrm{N}$ & $\# 1$ \\
\hline C9orf98 & 1608412610 & 9 & 9 & 24 & G & $<\mathrm{A}$ & $\mathrm{S}$ & $\mathrm{N}$ & $\# 1$ \\
\hline GFI1B & 1608580179 & 9 & 6 & 19 & $\mathrm{~T}$ & $<\mathrm{A}$ & NS & D & \#1 \\
\hline GFI1B & 1608580182 & 9 & 6 & 19 & G & $<\mathrm{T}$ & NS & NO & $\# 1$ \\
\hline $\mathrm{ABO}$ & 1608845331 & 9 & 7 & 39 & $\mathrm{C}$ & $<\mathrm{T}$ & NS & NR & \#4 \\
\hline $\mathrm{ABO}$ & 1608845366 & 9 & 7 & 43 & A & $<\mathrm{T}$ & NS & NR & \#4 \\
\hline SARDH & 1609287306 & 9 & 10 & 36 & G & $<\mathrm{A}$ & $\mathrm{S}$ & $\mathrm{N}$ & \#4 \\
\hline OLFM1 & 1610646021 & 9 & 2 & 93 & $\mathrm{~T}$ & $<\mathrm{C}$ & $\mathrm{S}$ & $\mathrm{N}$ & $\# 4$ \\
\hline PAEP & 1611120049 & 9 & 4 & 108 & $\mathrm{C}$ & $<\mathrm{A}$ & IS & $\mathrm{N}$ & $\# 4$ \\
\hline CACNA1B & 1613566398 & 9 & 28 & 22 & $\mathrm{G}$ & & $\mathrm{S}$ & $\mathrm{N}$ & $\# 3$ \\
\hline CACNA1B & 1613566402 & 9 & 28 & 24 & $\mathrm{~A}$ & $<$ & NS & $\mathrm{N}$ & $\# 3$ \\
\hline PFKP & 1617341440 & 10 & 9 & 103 & $\mathrm{C}$ & $<\mathrm{T}$ & $\mathrm{S}$ & $\mathrm{N}$ & $\# 4$ \\
\hline AKR1CL2 & 1619063490 & 10 & 2 & 31 & A & $<\mathrm{T}$ & NS & $\mathrm{D}$ & \#3 \\
\hline ITIH2 & 1621971113 & 10 & 16 & 62 & C & $<\mathrm{G}$ & NS & $\mathrm{N}$ & \#4 \\
\hline BEND7 & 1627671894 & 10 & 7 & 32 & $\mathrm{~T}$ & $<\mathrm{A}$ & $\mathrm{s}$ & $\mathrm{N}$ & $\# 4$ \\
\hline ARMETL1 & 1629060660 & 10 & 2 & 54 & $\mathrm{~T}$ & $<\mathrm{A}$ & NS & $\mathrm{D}$ & $\# 3$ \\
\hline CUBN & 1631072959 & 10 & 62 & 38 & $\mathrm{~T}$ & $<\mathrm{A}$ & $\mathrm{S}$ & $\mathrm{N}$ & \#3 \\
\hline CUBN & 1631276445 & 10 & 26 & 49 & $\mathrm{~T}$ & $<\mathrm{A}$ & NS & $\mathrm{N}$ & \#3 \\
\hline MRC1L1 & 1632130724 & 10 & 24 & 11 & $\mathrm{G}$ & $<\mathrm{T}$ & NS & $\mathrm{D}$ & \#4 \\
\hline PIP4K2A & 1637047251 & 10 & 6 & 18 & $\mathrm{~T}$ & $<\mathrm{A}$ & NS & $\mathrm{D}$ & $\# 3$ \\
\hline ARHGAP21 & 1639064800 & 10 & 25 & 109 & $\mathrm{C}$ & $<\mathrm{T}$ & NS & $\mathrm{N}$ & \#1 \\
\hline TMEM72 & 1656420929 & 10 & 5 & 46 & $\mathrm{C}$ & $<\mathrm{T}$ & $\mathrm{S}$ & $\mathrm{N}$ & \#4 \\
\hline ANUBL1 & 1657125773 & 10 & 5 & 19 & $\mathrm{C}$ & $<\mathrm{T}$ & $\mathrm{S}$ & $\mathrm{N}$ & \#4 \\
\hline ANXA8L2 & 1658602609 & 10 & 12 & 48 & $\mathrm{~T}$ & $<\mathrm{C}$ & $\mathrm{S}$ & $\mathrm{N}$ & \#4 \\
\hline AGAP9 & 1658906406 & 10 & 1 & 38 & $\mathrm{G}$ & $<\mathrm{T}$ & NS & $\mathrm{D}$ & $\# 4$ \\
\hline AGAP9 & 1658906463 & 10 & 1 & 30 & $\mathrm{~T}$ & $<\mathrm{G}$ & NS & $\mathrm{N}$ & \#4 \\
\hline $\mathrm{MSMB}^{*}$ & 1662146277 & 10 & 2 & 105 & A & $<\mathrm{G}$ & NS & $\mathrm{D}$ & \#1 \\
\hline PCDH15 & 1666172531 & 10 & 34 & 14 & $\mathrm{G}$ & $<\mathrm{T}$ & NS & $\mathrm{N}$ & $\# 2$ \\
\hline PCDH15* & 1666177751 & 10 & 32 & 70 & $\mathrm{~A}$ & $<\mathrm{G}$ & $\mathrm{S}$ & $\mathrm{N}$ & \#1 \\
\hline TMEM26 & 1673760923 & 10 & 6 & 22 & $\mathrm{C}$ & $<\mathrm{T}$ & NS & $\mathrm{N}$ & \#3 \\
\hline HKDC1 & 1681600842 & 10 & 12 & 23 & $\mathrm{C}$ & $<\mathrm{T}$ & $\mathrm{S}$ & $\mathrm{N}$ & \#4 \\
\hline ADAMTS14 & 1683108476 & 10 & 21 & 79 & $\mathrm{~A}$ & $<\mathrm{G}$ & NS & $\mathrm{N}$ & \#4 \\
\hline USP54 & 1685873867 & 10 & 15 & 93 & G & $<\mathrm{A}$ & NS & $\mathrm{N}$ & $\# 1$ \\
\hline
\end{tabular}




\begin{tabular}{|c|c|c|c|c|c|c|c|c|}
\hline DLG5 & 1690161340 & 10 & 23 & 67 & G & $<\mathrm{T}$ & NS & $\mathrm{N}$ \\
\hline FAM22B & 1692061925 & 10 & 7 & 25 & A & $<\mathrm{C}$ & $\mathrm{S}$ & $\mathrm{N}$ \\
\hline BMPR1A & 1699271849 & 10 & 9 & 83 & $\mathrm{C}$ & $<\mathrm{T}$ & $\mathrm{S}$ & $\mathrm{N}$ \\
\hline FAM25A & 1699372551 & 10 & 2 & 93 & A & $<\mathrm{G}$ & $\mathrm{S}$ & $\mathrm{N}$ \\
\hline MYOF & 1705731605 & 10 & 20 & 24 & G & $<\mathrm{C}$ & NS & $\mathrm{D}$ \\
\hline MYOF & 1705731606 & 10 & 20 & 24 & $\mathrm{C}$ & $<\mathrm{T}$ & $\mathrm{S}$ & $\mathrm{N}$ \\
\hline TLL2 & 1708736294 & 10 & 15 & 21 & $\mathrm{~T}$ & $<\mathrm{G}$ & NS & $\mathrm{N}$ \\
\hline TLL2 & 1708736297 & 10 & 15 & 22 & A & $<\mathrm{T}$ & NS & $\mathrm{D}$ \\
\hline MMS19* & 1709816131 & 10 & 18 & 118 & G & $<\mathrm{A}$ & $\mathrm{S}$ & $\mathrm{N}$ \\
\hline MMS19* & 1709816132 & 10 & 18 & 116 & $\mathrm{C}$ & $<\mathrm{A}$ & $\mathrm{S}$ & $\mathrm{N}$ \\
\hline BTRC & 1713888546 & 10 & 13 & 19 & $\mathrm{C}$ & $<\mathrm{G}$ & NS & $\mathrm{D}$ \\
\hline POLL & 1713935693 & 10 & 3 & 174 & G & $<\mathrm{T}$ & NS & $\mathrm{N}$ \\
\hline PNLIPRP1 & 1728959073 & 10 & 12 & 57 & $\mathrm{~T}$ & $<$ & NS & $\mathrm{D}$ \\
\hline DMBT1 & 1734932783 & 10 & 13 & 13 & G & $<\mathrm{T}$ & NS & NO \\
\hline DMBT1 & 1734934333 & 10 & 14 & 38 & A & $<\mathrm{T}$ & $\mathrm{S}$ & $\mathrm{N}$ \\
\hline DMBT1 & 1734942466 & 10 & 20 & 106 & $\mathrm{~T}$ & $<\mathrm{C}$ & $\mathrm{S}$ & $\mathrm{N}$ \\
\hline СTBP2 & 1737305458 & 10 & 1 & 13 & G & $<\mathrm{A}$ & S & $\mathrm{N}$ \\
\hline MMP21 & 1738045784 & 10 & 7 & 33 & G & $<\mathrm{A}$ & NS & $\mathrm{D}$ \\
\hline JAKMIP3 & 1744395894 & 10 & 10 & 74 & A & $<\mathrm{G}$ & NS & $\mathrm{N}$ \\
\hline C10orf93 & 1745195014 & 10 & 4 & 39 & G & $<\mathrm{A}$ & $\mathrm{S}$ & $\mathrm{N}$ \\
\hline $\mathrm{KNDC} 1$ & 1745437947 & 10 & 5 & 104 & $\mathrm{C}$ & $<\mathrm{T}$ & $\mathrm{s}$ & $\mathrm{N}$ \\
\hline SYCE1 & 1745809057 & 10 & 13 & 80 & $\mathrm{~T}$ & $<\mathrm{C}$ & NS & $\mathrm{N}$ \\
\hline FRG2B & 1745879355 & 10 & 4 & 105 & $\mathrm{C}$ & $<\mathrm{T}$ & $\mathrm{s}$ & $\mathrm{N}$ \\
\hline SCGB1C1 & 1746098936 & 11 & 2 & 28 & A & $<\mathrm{G}$ & $\mathrm{s}$ & $\mathrm{N}$ \\
\hline B4GALNT4 & 1746279024 & 11 & 8 & 44 & G & $<\mathrm{A}$ & $\mathrm{s}$ & $\mathrm{N}$ \\
\hline CHID1 & 1746775660 & 11 & 11 & 37 & G & $<\mathrm{A}$ & NS & $\mathrm{N}$ \\
\hline MUC2 & 1746998500 & 11 & 30 & 33 & $\mathrm{C}$ & $<\mathrm{G}$ & $\mathrm{s}$ & $\mathrm{N}$ \\
\hline MUC2 & 1746998519 & 11 & 30 & 39 & $\mathrm{C}$ & $<\mathrm{A}$ & $\mathrm{S}$ & $\mathrm{N}$ \\
\hline MUC5AC & 1747163509 & 11 & 40 & 33 & A & $<\mathrm{G}$ & $\mathrm{S}$ & $\mathrm{N}$ \\
\hline MUC5AC & 1747176291 & 11 & 50 & 197 & G & $<\mathrm{T}$ & NS & $\mathrm{N}$ \\
\hline MUC5AC & 1747183167 & 11 & 59 & 41 & G & $<\mathrm{A}$ & NS & $\mathrm{N}$ \\
\hline KRTAP5-3 & 1747534374 & 11 & 1 & 73 & $\mathrm{C}$ & $<\mathrm{T}$ & NS & $\mathrm{N}$ \\
\hline TNNT3 & 1747860451 & 11 & 10 & 88 & $\mathrm{C}$ & $<\mathrm{A}$ & NS & $\mathrm{D}$ \\
\hline ART1 & 1749586329 & 11 & 2 & 22 & A & $<\mathrm{G}$ & S & $\mathrm{N}$ \\
\hline DCHS1 & 1752558224 & 11 & 6 & 111 & $\mathrm{C}$ & $<\mathrm{T}$ & NS & D \\
\hline SOX6 & 1761982617 & 11 & 9 & 24 & $\mathrm{~T}$ & $<\mathrm{C}$ & $\mathrm{S}$ & $\mathrm{N}$ \\
\hline
\end{tabular}




\begin{tabular}{|c|c|c|c|c|c|c|c|c|c|}
\hline SAAL1 & 1764013726 & 11 & 9 & 29 & $\mathrm{~T}$ & $<\mathrm{C}$ & NS & $\mathrm{N}$ & \#4 \\
\hline SAAL1 & 1764016154 & 11 & 7 & 38 & $\mathrm{~T}$ & $<\mathrm{A}$ & NS & $\mathrm{N}$ & \#4 \\
\hline MRGPRX3 & 1764064511 & 11 & 1 & 277 & G & $<\mathrm{A}$ & $\mathrm{S}$ & NO & $\# 1$ \\
\hline MRGPRX3 & 1764064669 & 11 & 1 & 70 & $\mathrm{~T}$ & $<\mathrm{C}$ & NS & $\mathrm{D}$ & $\# 4$ \\
\hline NAV2 & 1765806846 & 11 & 5 & 40 & G & $<\mathrm{A}$ & $\mathrm{S}$ & $\mathrm{N}$ & \#4 \\
\hline NAV2 & 1765972037 & 11 & 14 & 74 & A & $<\mathrm{G}$ & NS & D & $\# 3$ \\
\hline FANCF & 1768551685 & 11 & 1 & 24 & $\mathrm{C}$ & $<\mathrm{T}$ & NS & $\mathrm{N}$ & $\# 3$ \\
\hline SLC5A12 & 1772648382 & 11 & 1 & 29 & $\mathrm{C}$ & $<\mathrm{A}$ & NS & NO & $\# 3$ \\
\hline SLC5A12 & 1772648383 & 11 & 1 & 29 & $\mathrm{C}$ & $<\mathrm{A}$ & NS & $\mathrm{N}$ & \#3 \\
\hline MPPED2 & 1776462933 & 11 & 2 & 21 & $\mathrm{G}$ & $<\mathrm{T}$ & $\mathrm{S}$ & $\mathrm{N}$ & \#3 \\
\hline MPPED2 & 1776462935 & 11 & 2 & 20 & $\mathrm{C}$ & $<\mathrm{G}$ & NS & $\mathrm{N}$ & \#3 \\
\hline ZNF408 & 1792629936 & 11 & 4 & 54 & $\mathrm{~T}$ & $<\mathrm{A}$ & NS & $\mathrm{N}$ & $\# 2$ \\
\hline GLYAT & 1800978539 & 11 & 3 & 36 & G & & NS & $\mathrm{D}$ & $\# 3$ \\
\hline PGA3 & 1803475551 & 11 & 6 & 166 & $\mathrm{~T}$ & $<c$ & NS & $\mathrm{D}$ & \#4 \\
\hline AHNAK & 1804789193 & 11 & 3 & 55 & $\mathrm{~T}$ & $<\mathrm{C}$ & $\mathrm{NS}$ & $\mathrm{N}$ & \#4 \\
\hline SIPA1 & 1807916289 & 11 & 15 & 12 & G & $<\mathrm{T}$ & $\mathrm{S}$ & $\mathrm{N}$ & \#1 \\
\hline CATSPER1 & 1808286286 & 11 & 7 & 52 & C & $<\mathrm{T}$ & $\mathrm{NS}$ & $\mathrm{N}$ & $\# 4$ \\
\hline RBM4B & 1808942484 & 11 & 1 & 34 & A & $<\mathrm{G}$ & NS & $\mathrm{D}$ & \#3 \\
\hline TPCN2 & 1811349628 & 11 & 19 & 316 & $\mathrm{~T}$ & $<\mathrm{C}$ & $\mathrm{NS}$ & $\mathrm{N}$ & \#4 \\
\hline FADD & 1812550653 & 11 & 2 & 45 & $\mathrm{G}$ & $<\mathrm{T}$ & $\mathrm{NS}$ & $\mathrm{N}$ & $\# 2$ \\
\hline C11orf30 & 1818755429 & 11 & 19 & 54 & $\mathrm{~T}$ & $<\mathrm{C}$ & $\mathrm{s}$ & $\mathrm{N}$ & $\# 4$ \\
\hline GDPD4 & 1819477756 & 11 & 0 & 38 & $\mathrm{C}$ & $<\mathrm{T}$ & NS & $\mathrm{D}$ & \#3 \\
\hline ALG8 & 1820310415 & 11 & 13 & 26 & G & $<\mathrm{A}$ & $\mathrm{S}$ & $\mathrm{N}$ & \#3 \\
\hline GAB2 & 1820434380 & 11 & 5 & 79 & A & $<\mathrm{G}$ & $\mathrm{S}$ & $\mathrm{N}$ & \#4 \\
\hline FAT3 & 1835072125 & 11 & 17 & 53 & G & $<\mathrm{T}$ & NS & $\mathrm{N}$ & \#4 \\
\hline PANX1 & 1836411162 & 11 & 4 & 21 & $\mathrm{C}$ & $<\mathrm{A}$ & NS & $\mathrm{N}$ & $\# 3$ \\
\hline PIWIL4 & 1836824979 & 11 & 9 & 76 & G & $<\mathrm{C}$ & NS & $\mathrm{N}$ & \#4 \\
\hline CWC15 & 1837197727 & 11 & 5 & 30 & A & $<\mathrm{G}$ & NS & NR & $\# 2$ \\
\hline TMEM133 & 1843211533 & 11 & 1 & 84 & A & $<\mathrm{C}$ & NS & $\mathrm{N}$ & \#4 \\
\hline TRPC6* & 1843723722 & 11 & 2 & 65 & $\mathrm{C}$ & $<\mathrm{T}$ & $\mathrm{S}$ & $\mathrm{N}$ & $\# 1$ \\
\hline TMEM123 & 1844621025 & 11 & 3 & 11 & $\mathrm{G}$ & $<\mathrm{A}$ & NS & $\mathrm{D}$ & $\# 2$ \\
\hline $\mathrm{ZC} 3 \mathrm{H} 12 \mathrm{C}$ & 1852355676 & 11 & 2 & 23 & $\mathrm{G}$ & $<\mathrm{T}$ & NS & $\mathrm{N}$ & \#3 \\
\hline LAYN & 1853779209 & 11 & 7 & 40 & G & $<\mathrm{A}$ & NS & $\mathrm{N}$ & $\# 2$ \\
\hline ZW10 & 1855955650 & 11 & 15 & 49 & A & $<\mathrm{C}$ & NS & $\mathrm{N}$ & $\# 3$ \\
\hline CEP164 & 1859631014 & 11 & 31 & 58 & G & $<\mathrm{T}$ & $\mathrm{S}$ & $\mathrm{N}$ & \#4 \\
\hline DSCAML1 & 1859657009 & 11 & 25 & 62 & G & $<\mathrm{A}$ & $\mathrm{S}$ & $\mathrm{N}$ & \#4 \\
\hline
\end{tabular}




\begin{tabular}{|c|c|c|c|c|c|c|c|c|c|}
\hline DSCAML1 & 1859751449 & 11 & 4 & 51 & G & $<\mathrm{T}$ & NS & $\mathrm{N}$ & $\# 4$ \\
\hline IL10RA & 1860212261 & 11 & 4 & 49 & $\mathrm{~A}$ & $<\mathrm{G}$ & $\mathrm{S}$ & $\mathrm{N}$ & $\# 4$ \\
\hline TMPRSS4 & 1860336319 & 11 & 12 & 79 & $\mathrm{C}$ & $<\mathrm{T}$ & NS & $\mathrm{D}$ & $\# 4$ \\
\hline BCL9L & 1861117811 & 11 & 8 & 14 & $\mathrm{G}$ & $<\mathrm{T}$ & NS & $\mathrm{D}$ & $\# 1$ \\
\hline CCDC84 & 1861234319 & 11 & 10 & 45 & $\mathrm{C}$ & $<\mathrm{G}$ & $\mathrm{S}$ & $\mathrm{N}$ & $\# 2$ \\
\hline ZNF202 & 1865948687 & 11 & 2 & 14 & $\mathrm{C}$ & $<\mathrm{A}$ & NS & JO & $\# 3$ \\
\hline HSN2 & 1878252896 & 12 & 1 & 49 & $\mathrm{~T}$ & $<\mathrm{C}$ & $\mathrm{S}$ & $\mathrm{N}$ & $\# 1$ \\
\hline VWF & 1883406765 & 12 & 25 & 19 & $\mathrm{~T}$ & $<\mathrm{C}$ & NS & $\mathrm{D}$ & $\# 2$ \\
\hline ACSM4 & 1884701983 & 12 & 11 & 32 & G & $<\mathrm{T}$ & NS & $\mathrm{D}$ & $\# 3$ \\
\hline PRB2 & 1888770834 & 12 & 3 & 58 & $\mathrm{~T}$ & $<\mathrm{C}$ & $\mathrm{s}$ & $\mathrm{N}$ & $\# 4$ \\
\hline PRB2 & 1888770905 & 12 & 3 & 100 & G & $<\mathrm{T}$ & NS & $\mathrm{N}$ & $\# 2$ \\
\hline PRB2 & 1888771519 & 12 & 3 & 100 & $\mathrm{C}$ & $<\mathrm{T}$ & NS & $\mathrm{N}$ & $\# 4$ \\
\hline PIK3C2G & 1895940574 & 12 & 25 & 19 & $\mathrm{C}$ & & NS & $\mathrm{D}$ & $\# 3$ \\
\hline SLCO1C1 & 1898110764 & 12 & 9 & 41 & A & - & $\mathrm{S}$ & $\mathrm{N}$ & $\# 4$ \\
\hline ARID2 & 1920469949 & 12 & 15 & 35 & $\mathrm{~T}$ & $<\mathrm{C}$ & $\mathrm{s}$ & $\mathrm{N}$ & $\# 3$ \\
\hline AMIGO2 & 1921696779 & 12 & 1 & 29 & C & $<\mathrm{A}$ & NS & $\mathrm{N}$ & $\# 3$ \\
\hline KRT86 & 1926923866 & 12 & 5 & 158 & $\mathrm{G}$ & $<\mathrm{A}$ & NS & $\mathrm{N}$ & $\# 4$ \\
\hline KRT86 & 1926923877 & 12 & 5 & 167 & $\mathrm{C}$ & $<\mathrm{G}$ & $\mathrm{S}$ & $\mathrm{N}$ & $\# 4$ \\
\hline KRT2 & 1927263989 & 12 & 9 & 24 & $\mathrm{C}$ & $<\mathrm{A}$ & NS & $\mathrm{D}$ & $\# 3$ \\
\hline KRT2 & 1927263991 & 12 & 9 & 24 & $\mathrm{C}$ & $<\mathrm{A}$ & NS & $\mathrm{D}$ & $\# 3$ \\
\hline NCKAP1L & 1929139765 & 12 & 18 & 111 & $\mathrm{C}$ & $<\mathrm{T}$ & $\mathrm{S}$ & $\mathrm{N}$ & $\# 4$ \\
\hline RDH16 & 1931575957 & 12 & & 144 & $\mathrm{G}$ & $<\mathrm{T}$ & NS & $\mathrm{D}$ & $\# 2$ \\
\hline LRP1 & 1931814492 & 12 & 54 & 29 & $\mathrm{C}$ & $<\mathrm{T}$ & $\mathrm{S}$ & $\mathrm{N}$ & $\# 4$ \\
\hline LRIG3 & 1933499258 & 12 & 13 & 28 & $\mathrm{~T}$ & $<\mathrm{A}$ & NS & $\mathrm{D}$ & $\# 3$ \\
\hline TMEM5 & 1938398588 & 12 & 1 & 72 & G & $<\mathrm{A}$ & $\mathrm{S}$ & $\mathrm{N}$ & $\# 4$ \\
\hline RASSF9 & 1960454767 & 12 & 1 & 16 & $\mathrm{~T}$ & $<\mathrm{A}$ & NS & NO & $\# 3$ \\
\hline C12orf12 & 1965572650 & 12 & 1 & 74 & $\mathrm{C}$ & $<\mathrm{A}$ & NS & $\mathrm{N}$ & $\# 3$ \\
\hline NUP37 & 1976695411 & 12 & 7 & 27 & $\mathrm{~A}$ & $<\mathrm{C}$ & NS & $\mathrm{D}$ & $\# 3$ \\
\hline USP30 & 1983694022 & 12 & 8 & 37 & $\mathrm{C}$ & $<\mathrm{T}$ & $\mathrm{S}$ & $\mathrm{N}$ & $\# 4$ \\
\hline C12orf51 & 1986840857 & 12 & 36 & 47 & $\mathrm{~T}$ & $<\mathrm{C}$ & $\mathrm{S}$ & $\mathrm{N}$ & $\# 2$ \\
\hline DDX54 & 1987789501 & 12 & 7 & 85 & G & $<\mathrm{A}$ & $\mathrm{S}$ & $\mathrm{N}$ & $\# 4$ \\
\hline PLBD2 & 1987987438 & 12 & 5 & 27 & $\mathrm{C}$ & $<\mathrm{T}$ & NS & NO & $\# 3$ \\
\hline SDSL & 1988046794 & 12 & 4 & 40 & G & $<\mathrm{T}$ & NS & $\mathrm{N}$ & $\# 3$ \\
\hline MED13L & 1990588227 & 12 & 24 & 18 & $\mathrm{C}$ & $<\mathrm{A}$ & NS & $\mathrm{D}$ & $\# 3$ \\
\hline CIT & 1994346828 & 12 & 24 & 71 & $\mathrm{~T}$ & $<\mathrm{C}$ & $\mathrm{S}$ & $\mathrm{N}$ & $\# 4$ \\
\hline ORAI1 & 1996254022 & 12 & 2 & 53 & $\mathrm{C}$ & $<\mathrm{T}$ & $\mathrm{S}$ & $\mathrm{N}$ & $\# 4$ \\
\hline
\end{tabular}




\begin{tabular}{|c|c|c|c|c|c|c|c|c|c|}
\hline B3GNT4 & 1996814014 & 12 & 1 & 20 & $\mathrm{C}$ & $<\mathrm{G}$ & NS & $\mathrm{N}$ & $\# 4$ \\
\hline CLIP1 & 1996970528 & 12 & 4 & 25 & G & $<\mathrm{A}$ & $\mathrm{S}$ & $\mathrm{N}$ & $\# 2$ \\
\hline SBNO1 & 1997954707 & 12 & 3 & 23 & $\mathrm{~T}$ & $<\mathrm{C}$ & NS & $\mathrm{N}$ & $\# 3$ \\
\hline SETD8 & 1998000144 & 12 & 3 & 24 & $\mathrm{C}$ & $<\mathrm{T}$ & $\mathrm{S}$ & $\mathrm{N}$ & $\# 2$ \\
\hline GPR133 & 2005609817 & 12 & 9 & 29 & A & $<\mathrm{G}$ & $\mathrm{S}$ & $\mathrm{N}$ & \#3 \\
\hline POLE & 2007276853 & 12 & 12 & 66 & $\mathrm{~T}$ & $<\mathrm{C}$ & NS & & $\# 3$ \\
\hline PGAM5 & 2007320186 & 12 & 6 & 59 & $\mathrm{C}$ & $<\mathrm{T}$ & NS & $\mathrm{N}$ & $\# 4$ \\
\hline TPTE2 & 2008847341 & 13 & 18 & 64 & A & $<\mathrm{G}$ & $\mathrm{S}$ & $\mathrm{N}$ & $\# 4$ \\
\hline TPTE2 & 2008847342 & 13 & 18 & 61 & $\mathrm{~T}$ & $<\mathrm{A}$ & NS & $\mathrm{N}$ & $\# 4$ \\
\hline PARP4 & 2013890787 & 13 & 15 & 79 & G & $<\mathrm{A}$ & $\mathrm{S}$ & $\mathrm{N}$ & $\# 1$ \\
\hline SLC7A1 & 2018953795 & 13 & 2 & 58 & G & $<\mathrm{C}$ & S & $\mathrm{N}$ & \#4 \\
\hline NBEA & 2024476994 & 13 & 7 & 190 & $\mathrm{~T}$ & $<\mathrm{C}$ & $\mathrm{S}$ & $\mathrm{N}$ & $\# 4$ \\
\hline DCLK1 & 2025231759 & 13 & 11 & 53 & G & $<$ & $\mathrm{S}$ & $\mathrm{N}$ & $\# 4$ \\
\hline KBTBD6 & 2030552300 & 13 & 1 & 29 & $\mathrm{C}$ & $<\mathrm{T}$ & NS & $\mathrm{N}$ & $\# 4$ \\
\hline MED4 & 2037507221 & 13 & 3 & 16 & $\mathrm{~T}$ & $<\mathrm{A}$ & NS & $\mathrm{D}$ & $\# 3$ \\
\hline RB1 & 2037880563 & 13 & 20 & 49 & G & $<\mathrm{A}$ & $\mathrm{S}$ & $\mathrm{D}$ & $\# 1$ \\
\hline RCBTB1 & 2038988090 & 13 & 1 & 88 & G & $<\mathrm{A}$ & $\mathrm{S}$ & $\mathrm{N}$ & $\# 4$ \\
\hline PCDH9 & 2056646696 & 13 & 1 & 23 & $\mathrm{~T}$ & $<\mathrm{A}$ & NS & $\mathrm{NO}$ & $\# 3$ \\
\hline PCDH9 & 2056646999 & 13 & 1 & 39 & $\mathrm{C}$ & $<\mathrm{A}$ & NS & $\mathrm{N}$ & $\# 3$ \\
\hline KLF12 & 2063234134 & 13 & 4 & 43 & $\mathrm{C}$ & $<\mathrm{G}$ & NS & $\mathrm{N}$ & \#3 \\
\hline COL4A1 & 2099524302 & 13 & 7 & 135 & $\mathrm{~T}$ & $<\mathrm{A}$ & $\mathrm{S}$ & $\mathrm{N}$ & \#4 \\
\hline C13orf16 & 2100677265 & 13 & 2 & 112 & $\mathrm{C}$ & $<\mathrm{T}$ & $\mathrm{S}$ & $\mathrm{N}$ & \#4 \\
\hline ATP11A & 2102082860 & 13 & 29 & 44 & $\mathrm{~T}$ & $<\mathrm{C}$ & $\mathrm{S}$ & $\mathrm{N}$ & \#4 \\
\hline RASA3 & 2103108926 & 13 & 21 & 105 & $\mathrm{C}$ & $<\mathrm{T}$ & $\mathrm{S}$ & $\mathrm{N}$ & \#4 \\
\hline POTEG & 2104010042 & 14 & 1 & 119 & $\mathrm{C}$ & $<\mathrm{T}$ & NS & $\mathrm{N}$ & \#4 \\
\hline P704P & 2104476800 & 14 & 1 & 60 & $\mathrm{C}$ & $<\mathrm{T}$ & $\mathrm{S}$ & $\mathrm{N}$ & \#4 \\
\hline NDRG2 & 2105942492 & 14 & 15 & 51 & G & $<\mathrm{C}$ & NS & $\mathrm{D}$ & \#4 \\
\hline HAUS4 & 2107873469 & 14 & 7 & 11 & G & $<\mathrm{T}$ & $\mathrm{S}$ & $\mathrm{N}$ & \#2 \\
\hline HOMEZ & 2108202700 & 14 & 2 & 14 & A & $<\mathrm{T}$ & NS & $\mathrm{N}$ & \#3 \\
\hline DHRS4 & 2108891643 & 14 & 4 & 116 & $\mathrm{C}$ & $<\mathrm{T}$ & NS & NO & $\# 4$ \\
\hline DHRS4L2 & 2108914889 & 14 & 1 & 132 & G & $<\mathrm{T}$ & NS & $\mathrm{D}$ & $\# 3$ \\
\hline DHRS4L2 & 2108916099 & 14 & 2 & 35 & G & $<\mathrm{A}$ & $\mathrm{S}$ & $\mathrm{N}$ & \#4 \\
\hline GZMH & 2109533483 & 14 & 3 & 196 & G & $<\mathrm{C}$ & NS & $\mathrm{N}$ & $\# 4$ \\
\hline GZMH & 2109533541 & 14 & 3 & 38 & C & $<\mathrm{A}$ & NS & $\mathrm{D}$ & $\# 3$ \\
\hline C14orf182 & 2134929075 & 14 & 1 & 27 & A & $<\mathrm{T}$ & NS & $\mathrm{D}$ & \#3 \\
\hline MAP4K5 & 2135379887 & 14 & 13 & 28 & $\mathrm{G}$ & $<\mathrm{A}$ & NS & $\mathrm{D}$ & \#3 \\
\hline
\end{tabular}




\begin{tabular}{|c|c|c|c|c|c|c|c|c|c|}
\hline PPM1A & 2145206418 & 14 & 2 & 80 & G & $<\mathrm{T}$ & NS & $\mathrm{N}$ & \#3 \\
\hline SYNE2 & 2148878083 & 14 & 7 & 85 & $\mathrm{~A}$ & $<\mathrm{G}$ & NS & $\mathrm{D}$ & $\# 1$ \\
\hline PLEKHG3 & 2149666422 & 14 & 14 & 36 & $\mathrm{C}$ & $<\mathrm{A}$ & NS & $\mathrm{D}$ & $\# 2$ \\
\hline PLEKHG3 & 2149666423 & 14 & 14 & 38 & $\mathrm{C}$ & $<\mathrm{T}$ & NS & $\mathrm{D}$ & $\# 2$ \\
\hline GPHN & 2151839368 & 14 & 6 & 21 & G & $<\mathrm{A}$ & $\mathrm{S}$ & $\mathrm{N}$ & $\# 3$ \\
\hline SIPA1L1 & 2156512152 & 14 & 1 & 22 & $\mathrm{~A}$ & $<\mathrm{T}$ & $\mathrm{S}$ & $\mathrm{N}$ & $\# 3$ \\
\hline DIO2 & 2165125971 & 14 & 3 & 63 & $\mathrm{C}$ & $<\mathrm{T}$ & $\mathrm{S}$ & $\mathrm{N}$ & $\# 3$ \\
\hline FLRT2 & 2170544935 & 14 & 1 & 20 & G & $<\mathrm{T}$ & NS & $\mathrm{N}$ & $\# 3$ \\
\hline DDX24 & 2178978135 & 14 & 6 & 101 & A & $<\mathrm{C}$ & NS & $\mathrm{D}$ & $\# 2$ \\
\hline BEGAIN & 2185466810 & 14 & 4 & 97 & $\mathrm{~T}$ & $<\mathrm{C}$ & $\mathrm{S}$ & $\mathrm{N}$ & $\# 4$ \\
\hline C14orf73 & 2188025488 & 14 & 2 & 16 & $\mathrm{~T}$ & $<\mathrm{G}$ & $\mathrm{S}$ & $\mathrm{N}$ & $\# 2$ \\
\hline TMEM179 & 2189527477 & 14 & 1 & 28 & $\mathrm{C}$ & $<\mathrm{G}$ & $\mathrm{N}$ & $\mathrm{N}$ & $\# 2$ \\
\hline ADSSL1 & 2189666079 & 14 & 10 & 107 & G & & NS & $\mathrm{D}$ & \#2 \\
\hline AHNAK2 & 2189862548 & 14 & 7 & 57 & G & $<\mathrm{A}$ & $\mathrm{S}$ & $\mathrm{N}$ & \#4 \\
\hline AHNAK2 & 2189862844 & 14 & 7 & 26 & A & $<\mathrm{C}$ & NS & $\mathrm{N}$ & \#4 \\
\hline LOC 727832 & 2192485885 & 15 & 8 & 14 & A & $<\mathrm{G}$ & NS & $\mathrm{N}$ & $\# 1$ \\
\hline C15orf2 & 2196518354 & 15 & 1 & 17 & C & $<\mathrm{G}$ & $\mathrm{S}$ & $\mathrm{N}$ & $\# 3$ \\
\hline GOLGA8G & 2200364560 & 15 & 8 & 34 & $\mathrm{~T}$ & $<\mathrm{A}$ & NS & $\mathrm{D}$ & $\# 3$ \\
\hline GOLGA8G & 2200368232 & 15 & 3 & 23 & $\mathrm{~T}$ & $<\mathrm{G}$ & NS & $\mathrm{D}$ & $\# 4$ \\
\hline CHRNA7 & 2203996850 & 15 & 7 & 134 & G & $<\mathrm{A}$ & $\mathrm{S}$ & $\mathrm{N}$ & $\# 4$ \\
\hline RYR3 & 2205498123 & 15 & 33 & 72 & $\mathrm{~T}$ & $<\mathrm{G}$ & $\mathrm{S}$ & $\mathrm{N}$ & $\# 4$ \\
\hline RYR3 & 2205507714 & 15 & 37 & 44 & $\mathrm{~T}$ & $<\mathrm{G}$ & NS & $\mathrm{D}$ & $\# 1$ \\
\hline SRP14 & 2211874811 & 15 & 5 & 170 & $\mathrm{G}$ & $<\mathrm{A}$ & $\mathrm{S}$ & $\mathrm{N}$ & \#4 \\
\hline STARD9 & 2214531200 & 15 & 23 & 19 & $\mathrm{~A}$ & $<\mathrm{T}$ & NS & $\mathrm{N}$ & $\# 3$ \\
\hline DMXL2 & 2223337705 & 15 & 18 & 32 & $\mathrm{~A}$ & $<\mathrm{G}$ & NS & $\mathrm{N}$ & $\# 4$ \\
\hline RNF111 & 2230919345 & 15 & 7 & 17 & $\mathrm{C}$ & $<\mathrm{T}$ & $\mathrm{S}$ & $\mathrm{N}$ & $\# 3$ \\
\hline ANXA2 & 2232187466 & 15 & 12 & 29 & G & $<\mathrm{A}$ & NS & $\mathrm{D}$ & $\# 2$ \\
\hline ITGA11 & 2240170436 & 15 & 14 & 59 & G & $<\mathrm{A}$ & $\mathrm{S}$ & $\mathrm{N}$ & $\# 4$ \\
\hline GOLGA6B & 2244497986 & 15 & 4 & 49 & G & $<\mathrm{A}$ & $\mathrm{S}$ & $\mathrm{N}$ & $\# 4$ \\
\hline GOLGA6 & 2245910065 & 15 & 15 & 18 & G & $<\mathrm{T}$ & NS & $\mathrm{N}$ & \#1 \\
\hline CYP1A2 & 2246588818 & 15 & 1 & 41 & A & $<\mathrm{G}$ & NS & $\mathrm{D}$ & $\# 3$ \\
\hline GOLGA6C & 2247104814 & 15 & 11 & 23 & A & $<\mathrm{T}$ & NS & $\mathrm{N}$ & \#4 \\
\hline GOLGA6C & 2247104889 & 15 & 11 & 35 & G & $<\mathrm{A}$ & NS & $\mathrm{N}$ & $\# 4$ \\
\hline GOLGA6C & 2247106801 & 15 & 13 & 22 & G & $<\mathrm{A}$ & NS & $\mathrm{N}$ & $\# 2$ \\
\hline CSPG4 & 2247528218 & 15 & 3 & 39 & G & $<\mathrm{A}$ & NS & $\mathrm{D}$ & $\# 2$ \\
\hline SGK269 & 2248972147 & 15 & 3 & 11 & G & $<\mathrm{C}$ & NS & $\mathrm{N}$ & \#3 \\
\hline
\end{tabular}




\begin{tabular}{|c|c|c|c|c|c|c|c|c|c|}
\hline KIAA1024 & 2251295855 & 15 & 1 & 14 & $\mathrm{C}$ & $<\mathrm{A}$ & NS & $\mathrm{N}$ & \#3 \\
\hline AP3B2 & 2254828471 & 15 & 20 & 55 & A & $<\mathrm{G}$ & S & $\mathrm{N}$ & $\# 1$ \\
\hline LOC100288732 & 2260124462 & 15 & 5 & 24 & G & $<\mathrm{C}$ & NS & NR & $\# 3$ \\
\hline LOC100288732 & 2260124464 & 15 & 5 & 27 & $\mathrm{~T}$ & $<\mathrm{A}$ & NS & NR & $\# 3$ \\
\hline KIF7 & 2261638402 & 15 & 3 & 16 & $\mathrm{~T}$ & $<\mathrm{G}$ & NS & $\mathrm{D}$ & $\# 2$ \\
\hline SEMA4B & 2262210365 & 15 & 5 & 49 & $\mathrm{G}$ & $<\mathrm{A}$ & NS & $\mathrm{N}$ & $\# 4$ \\
\hline FURIN & 2262865860 & 15 & 3 & 115 & $\mathrm{~T}$ & $<\mathrm{G}$ & NS & D & $\# 4$ \\
\hline MEF2A & 2271698884 & 15 & 9 & 28 & A & $<\mathrm{C}$ & NS & $\mathrm{N}$ & $\# 3$ \\
\hline ADAMTS17 & 2271960760 & 15 & 22 & 65 & $\mathrm{~T}$ & $<\mathrm{C}$ & NS & $\mathrm{N}$ & $\# 4$ \\
\hline HBA2 & 2274131104 & 16 & 3 & 26 & $\mathrm{~T}$ & $<\mathrm{G}$ & $\mathrm{S}$ & $\mathrm{N}$ & $\# 3$ \\
\hline PDIA2 & 2274242118 & 16 & 2 & 51 & G & $<\mathrm{C}$ & S & $\mathrm{N}$ & $\# 4$ \\
\hline JMJD8 & 2274641266 & 16 & 4 & 23 & $\mathrm{~T}$ & $<\mathrm{A}$ & NS & $\mathrm{N}$ & $\# 3$ \\
\hline PRSS22 & 2276813235 & 16 & 4 & 38 & $\mathrm{C}$ & $<\mathrm{T}$ & NS & $\mathrm{N}$ & $\# 4$ \\
\hline CLDN9 & 2276971003 & 16 & 1 & 43 & $\mathrm{C}$ & $<$ & $\mathrm{s}$ & $\mathrm{N}$ & \#1 \\
\hline ALG1 & 2279037309 & 16 & 9 & 58 & $\mathrm{~T}$ & $<\mathrm{C}$ & S & $\mathrm{N}$ & \#4 \\
\hline TMEM114 & 2282529635 & 16 & 1 & 33 & A & $<\mathrm{T}$ & NS & $\mathrm{D}$ & \#4 \\
\hline TEKT5 & 2284677496 & 16 & 5 & 142 & $\mathrm{~T}$ & $<\mathrm{C}$ & NS & $\mathrm{N}$ & $\# 4$ \\
\hline NOMO2 & 2292457809 & 16 & 10 & 19 & $\mathrm{~T}$ & $<\mathrm{A}$ & NS & $\mathrm{N}$ & $\# 4$ \\
\hline TMC5 & 2293409286 & 16 & 16 & 94 & $\mathrm{C}$ & $<\mathrm{T}$ & NS & NO & $\# 1$ \\
\hline ACSM5* & 2294342868 & 16 & 5 & 162 & $\mathrm{C}$ & $<\mathrm{G}$ & NS & $\mathrm{N}$ & $\# 1$ \\
\hline ACSM5 & 2294348591 & 16 & & 60 & $\mathrm{C}$ & $<\mathrm{G}$ & NS & $\mathrm{D}$ & $\# 4$ \\
\hline NPIPL3 & 2295321650 & 16 & 8 & 32 & A & $<\mathrm{G}$ & $\mathrm{s}$ & $\mathrm{N}$ & $\# 4$ \\
\hline OTOA & 2295663836 & 16 & 23 & 35 & $\mathrm{C}$ & $<\mathrm{T}$ & NS & $\mathrm{D}$ & \#4 \\
\hline VWA3A & 2296051856 & 16 & 20 & 40 & $\mathrm{C}$ & $<\mathrm{T}$ & NS & $\mathrm{N}$ & \#4 \\
\hline PRKCB & 2298073558 & 16 & 10 & 19 & $\mathrm{C}$ & $<\mathrm{T}$ & NS & NO & \#3 \\
\hline KIAA0556 & 2301696816 & 16 & 27 & 72 & G & $<\mathrm{A}$ & NS & $\mathrm{N}$ & $\# 4$ \\
\hline TUFM* & 2302763330 & 16 & 6 & 66 & $\mathrm{C}$ & $<\mathrm{A}$ & s & $\mathrm{N}$ & $\# 2$ \\
\hline INO80E & 2303915676 & 16 & 3 & 14 & A & $<\mathrm{G}$ & $\mathrm{S}$ & $\mathrm{N}$ & \#3 \\
\hline POL3S & 2305006549 & 16 & 3 & 23 & $\mathrm{~T}$ & $<\mathrm{C}$ & NS & $\mathrm{N}$ & $\# 4$ \\
\hline ERAF & 2305447472 & 16 & 2 & 18 & G & $<\mathrm{T}$ & S & $\mathrm{N}$ & $\# 4$ \\
\hline LOC100287647 & 2307849444 & 16 & 2 & 151 & G & $<\mathrm{A}$ & s & NR & \#1 \\
\hline $\mathrm{ABCC} 12$ & 2310775449 & 16 & 28 & 22 & $\mathrm{~T}$ & $<\mathrm{G}$ & NS & $\mathrm{D}$ & \#3 \\
\hline $\mathrm{ABCC} 12$ & 2310775450 & 16 & 28 & 22 & $\mathrm{C}$ & $<\mathrm{A}$ & NS & $\mathrm{D}$ & \#3 \\
\hline $\mathrm{ABCC} 12$ & 2310796723 & 16 & 19 & 112 & G & $<\mathrm{A}$ & S & $\mathrm{N}$ & $\# 1$ \\
\hline BRD7 & 2313015114 & 16 & 12 & 42 & A & $<\mathrm{C}$ & NS & NO & \#4 \\
\hline SALL1 & 2313830540 & 16 & 2 & 19 & A & $<\mathrm{C}$ & NS & D & \#3 \\
\hline
\end{tabular}




\begin{tabular}{|c|c|c|c|c|c|c|c|c|c|}
\hline CETP & 2319673630 & 16 & 14 & 59 & G & $<\mathrm{A}$ & NS & $\mathrm{N}$ & \#4 \\
\hline SETD6 & 2321208022 & 16 & 5 & 48 & G & $<\mathrm{A}$ & $\mathrm{s}$ & $\mathrm{N}$ & $\# 4$ \\
\hline CDH5 & 2329078423 & 16 & 2 & 105 & $\mathrm{C}$ & $<\mathrm{T}$ & $\mathrm{s}$ & $\mathrm{N}$ & $\# 4$ \\
\hline PDPR & 2332847939 & 16 & 17 & 49 & $\mathrm{C}$ & $<\mathrm{T}$ & $\mathrm{S}$ & $\mathrm{N}$ & $\# 4$ \\
\hline PKD1L2 & 2343889813 & 16 & 7 & 21 & $\mathrm{G}$ & $<\mathrm{A}$ & NS & NR & $\# 4$ \\
\hline MPHOSPH6 & 2344839892 & 16 & 5 & 61 & $\mathrm{G}$ & $<\mathrm{A}$ & $\mathrm{S}$ & & $\# 4$ \\
\hline CRISPLD2 & 2347537002 & 16 & 2 & 20 & $\mathrm{~A}$ & $<\mathrm{G}$ & NS & $\mathrm{N}$ & $\# 4$ \\
\hline FAM38A & 2351390771 & 16 & 35 & 14 & $\mathrm{C}$ & $<\mathrm{T}$ & NS & $\mathrm{N}$ & $\# 4$ \\
\hline FAM38A & 2351393656 & 16 & 33 & 16 & $\mathrm{~A}$ & $<\mathrm{C}$ & NS & $\mathrm{D}$ & $\# 1$ \\
\hline WDR81 & 2354433283 & 17 & 1 & 18 & G & $<\mathrm{A}$ & $\mathrm{S}$ & $\mathrm{N}$ & $\# 4$ \\
\hline WDR81 & 2354443084 & 17 & 10 & 67 & $\mathrm{C}$ & $<\mathrm{T}$ & $\mathrm{S}$ & $\mathrm{N}$ & $\# 4$ \\
\hline TSR1 & 2355042000 & 17 & 1 & 34 & $\mathrm{G}$ & $<\mathrm{A}$ & NS & $\mathrm{D}$ & $\# 3$ \\
\hline TRPV3 & 2356238371 & 17 & 7 & 155 & $\mathrm{C}$ & & $\mathrm{S}$ & $\mathrm{N}$ & $\# 4$ \\
\hline TRPV3 & 2356249176 & 17 & 4 & 23 & $\mathrm{~T}$ & & NS & $\mathrm{N}$ & \#4 \\
\hline ITGAE & 2356435127 & 17 & 24 & 49 & G & $<\mathrm{A}$ & NS & $\mathrm{D}$ & \#4 \\
\hline ITGAE & 2356463343 & 17 & 9 & 31 & G & $<\mathrm{A}$ & $\mathrm{S}$ & $\mathrm{D}$ & $\# 4$ \\
\hline ZZEF1 & 2356772099 & 17 & 28 & 13 & C & $<\mathrm{A}$ & NS & $\mathrm{N}$ & $\# 3$ \\
\hline GGT6 & 2357265990 & 17 & 1 & 13 & G & $<\mathrm{A}$ & NS & $\mathrm{N}$ & $\# 4$ \\
\hline CXCL16 & 2357444046 & 17 & 3 & 22 & $\mathrm{C}$ & $<\mathrm{T}$ & $\mathrm{S}$ & $\mathrm{N}$ & $\# 4$ \\
\hline TEKT1 & 2359518533 & 17 & 5 & 87 & G & $<\mathrm{A}$ & NS & $\mathrm{D}$ & $\# 4$ \\
\hline AMAC1L3 & 2360187655 & 17 & 2 & 35 & G & $<\mathrm{A}$ & NS & $\mathrm{N}$ & $\# 4$ \\
\hline AMAC1L3 & 2360187863 & 17 & 2 & 20 & G & $<\mathrm{A}$ & NS & $\mathrm{N}$ & $\# 4$ \\
\hline AMAC1L3 & 2360188508 & 17 & 2 & 179 & $\mathrm{~T}$ & $<\mathrm{C}$ & NS & $\mathrm{N}$ & $\# 4$ \\
\hline TP53* & 2360379830 & 17 & 6 & 38 & G & $<\mathrm{A}$ & NS & $\mathrm{D}$ & $\# 1$ \\
\hline MYH13 & 2363018801 & 17 & 28 & 98 & G & $<\mathrm{A}$ & $\mathrm{s}$ & $\mathrm{N}$ & $\# 4$ \\
\hline COX10 & 2366807730 & 17 & 4 & 62 & G & $<\mathrm{A}$ & $\mathrm{S}$ & $\mathrm{N}$ & $\# 4$ \\
\hline COX10 & 2366897810 & 17 & 6 & 105 & $\mathrm{C}$ & $<\mathrm{T}$ & $\mathrm{S}$ & $\mathrm{N}$ & $\# 4$ \\
\hline FAM18B2 & 2368251449 & 17 & 5 & 57 & A & $<\mathrm{G}$ & NS & $\mathrm{N}$ & $\# 4$ \\
\hline FAM18B2 & 2368259332 & 17 & 3 & 141 & A & $<\mathrm{C}$ & $\mathrm{S}$ & $\mathrm{N}$ & $\# 4$ \\
\hline TBC1D26 & 2368443097 & 17 & 3 & 80 & A & $<\mathrm{C}$ & NS & $\mathrm{D}$ & $\# 4$ \\
\hline TBC1D26 & 2368443106 & 17 & 3 & 90 & $\mathrm{~A}$ & $<\mathrm{G}$ & NS & $\mathrm{N}$ & $\# 4$ \\
\hline SHMT1 & 2371041192 & 17 & 7 & 30 & $\mathrm{C}$ & $<\mathrm{T}$ & $\mathrm{S}$ & $\mathrm{N}$ & $\# 3$ \\
\hline LGALS9C & 2371193284 & 17 & 4 & 95 & $\mathrm{C}$ & $<\mathrm{T}$ & $\mathrm{S}$ & $\mathrm{N}$ & $\# 4$ \\
\hline LGALS9C & 2371193294 & 17 & 4 & 83 & G & $<\mathrm{A}$ & NS & $\mathrm{N}$ & $\# 4$ \\
\hline ULK2 & 2372501833 & 17 & 19 & 21 & $\mathrm{G}$ & $<\mathrm{T}$ & $\mathrm{S}$ & $\mathrm{N}$ & \#3 \\
\hline KCNJ12 & 2374121073 & 17 & 1 & 157 & $\mathrm{G}$ & $<\mathrm{A}$ & NS & D & \#4 \\
\hline
\end{tabular}




\begin{tabular}{|c|c|c|c|c|c|c|c|c|c|}
\hline KCNJ12 & 2374121499 & 17 & 1 & 55 & $\mathrm{C}$ & $<\mathrm{T}$ & NS & $\mathrm{D}$ & $\# 4$ \\
\hline KCNJ12 & 2374121521 & 17 & 1 & 67 & G & $<\mathrm{C}$ & NS & $\mathrm{N}$ & $\# 4$ \\
\hline KIAA0100 & 2376657621 & 17 & 24 & 46 & $\mathrm{~A}$ & $<\mathrm{C}$ & NS & $\mathrm{N}$ & \#4 \\
\hline SUPT6H & 2376730797 & 17 & 36 & 14 & $\mathrm{C}$ & $<\mathrm{T}$ & $\mathrm{S}$ & $\mathrm{N}$ & $\# 1$ \\
\hline CCL8* & 2382349668 & 17 & 2 & 51 & A & $<\mathrm{G}$ & NS & $\mathrm{N}$ & $\# 1$ \\
\hline TBC1D3B & 2384202011 & 17 & 5 & 27 & $\mathrm{C}$ & $<\mathrm{T}$ & NS & D & $\# 4$ \\
\hline TBC1D3E & 2384401830 & 17 & 9 & 36 & A & $<\mathrm{T}$ & NS & D & $\# 4$ \\
\hline TBC1D3D & 2385938140 & 17 & 2 & 61 & A & $<\mathrm{G}$ & NS & $\mathrm{N}$ & $\# 4$ \\
\hline TBC1D3D & 2385940014 & 17 & 4 & 85 & $\mathrm{C}$ & $<\mathrm{A}$ & NS & $\mathrm{N}$ & $\# 4$ \\
\hline TBC1D3D & 2385940944 & 17 & 6 & 147 & G & $<\mathrm{A}$ & NS & $\mathrm{N}$ & $\# 4$ \\
\hline ERBB2 & 2387531879 & 17 & 17 & 51 & A & $<\mathrm{G}$ & NS & $\mathrm{N}$ & $\# 4$ \\
\hline TOP2A & 2388219990 & 17 & 9 & 19 & $\mathrm{~T}$ & $<\mathrm{C}$ & $\mathrm{S}$ & $\mathrm{N}$ & \#3 \\
\hline KRT25 & 2388559828 & 17 & 4 & 159 & G & $<$ & $\mathrm{S}$ & $\mathrm{N}$ & $\# 2$ \\
\hline KRT26 & 2388580603 & 17 & 1 & 18 & $\mathrm{~A}$ & $<7$ & $\mathrm{~S}$ & $\mathrm{~N}$ & \#3 \\
\hline KRT40 & 2388787496 & 17 & 6 & 48 & A & $<\mathrm{G}$ & $\mathrm{s}$ & $\mathrm{N}$ & $\# 4$ \\
\hline KRTAP3-2 & 2388808375 & 17 & 1 & 95 & $\mathrm{~T}$ & $<\mathrm{C}$ & NS & $\mathrm{N}$ & $\# 4$ \\
\hline KRTAP1-1 & 2388849768 & 17 & 1 & 37 & G & $<\mathrm{C}$ & NS & $\mathrm{D}$ & $\# 4$ \\
\hline KRTAP4-1 & 2388993082 & 17 & 2 & 33 & G & $<\mathrm{C}$ & NS & $\mathrm{N}$ & $\# 4$ \\
\hline KRTAP4-1 & 2388993086 & 17 & 2 & 30 & A & $<\mathrm{G}$ & S & $\mathrm{N}$ & $\# 4$ \\
\hline KRTAP9-4 & 2389058283 & 17 & 1 & 217 & $\mathrm{C}$ & $<\mathrm{T}$ & NS & $\mathrm{D}$ & $\# 4$ \\
\hline KRTAP9-4 & 2389058336 & 17 & 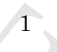 & 39 & A & $<\mathrm{C}$ & NS & $\mathrm{N}$ & \#3 \\
\hline KRTAP9-9 & 2389063986 & 17 & & 44 & A & $<\mathrm{C}$ & NS & $\mathrm{N}$ & $\# 4$ \\
\hline TUBG1 & 2390418855 & 17 & 10 & 14 & $\mathrm{C}$ & $<\mathrm{A}$ & $\mathrm{s}$ & $\mathrm{N}$ & $\# 1$ \\
\hline BRCA1 & 2390878791 & 17 & 13 & 31 & $\mathrm{C}$ & $<\mathrm{T}$ & NS & NO & $\# 3$ \\
\hline NAGS & 2391737375 & 17 & 6 & 45 & G & $<\mathrm{A}$ & NS & $\mathrm{N}$ & $\# 3$ \\
\hline CDK5RAP3 & 2395703185 & 17 & 3 & 61 & $\mathrm{~T}$ & $<\mathrm{G}$ & NS & $\mathrm{D}$ & $\# 2$ \\
\hline FAM117A & 2397445898 & 17 & 7 & 17 & G & $<\mathrm{A}$ & $\mathrm{s}$ & $\mathrm{N}$ & $\# 3$ \\
\hline ITGA3 & 2397801147 & 17 & 6 & 33 & $\mathrm{~T}$ & $<\mathrm{C}$ & $\mathrm{S}$ & $\mathrm{N}$ & $\# 4$ \\
\hline NOG & 2404324293 & 17 & 1 & 67 & A & $<\mathrm{C}$ & NS & $\mathrm{N}$ & $\# 3$ \\
\hline MTMR4 & 2406238200 & 17 & 6 & 13 & A & $<\mathrm{T}$ & NS & NO & $\# 3$ \\
\hline $\mathrm{CSH} 2$ & 2411602334 & 17 & 4 & 55 & C & $<\mathrm{T}$ & NS & $\mathrm{N}$ & $\# 4$ \\
\hline GH2 & 2411610386 & 17 & 4 & 18 & G & $<\mathrm{C}$ & NS & $\mathrm{N}$ & $\# 1$ \\
\hline TEX2 & 2411943294 & 17 & 1 & 21 & A & $<\mathrm{T}$ & NS & $\mathrm{D}$ & $\# 3$ \\
\hline COG1 & 2420849730 & 17 & 7 & 78 & C & $<\mathrm{T}$ & $\mathrm{S}$ & $\mathrm{N}$ & $\# 4$ \\
\hline GPR142* & 2422019077 & 17 & 3 & 57 & A & $<\mathrm{G}$ & NS & $\mathrm{D}$ & $\# 1$ \\
\hline UNK & 2423468317 & 17 & 14 & 19 & A & $<\mathrm{G}$ & $\mathrm{S}$ & $\mathrm{N}$ & $\# 4$ \\
\hline
\end{tabular}




\begin{tabular}{|c|c|c|c|c|c|c|c|c|c|}
\hline QRICH2 & 2423941144 & 17 & 4 & 44 & $\mathrm{~T}$ & $<\mathrm{G}$ & NS & $\mathrm{N}$ & $\# 4$ \\
\hline HRNBP3 & 2426764023 & 17 & 1 & 22 & $\mathrm{G}$ & $<\mathrm{A}$ & $\mathrm{S}$ & $\mathrm{N}$ & $\# 4$ \\
\hline CBX4 & 2427461157 & 17 & 5 & 66 & $\mathrm{C}$ & $<\mathrm{T}$ & NS & $\mathrm{N}$ & $\# 2$ \\
\hline RNF213 & 2427979649 & 17 & 9 & 73 & $\mathrm{G}$ & $<\mathrm{A}$ & $\mathrm{S}$ & $\mathrm{N}$ & $\# 4$ \\
\hline MYL12A & 2434375274 & 18 & 1 & 40 & $\mathrm{G}$ & $<\mathrm{A}$ & NS & $\mathrm{N}$ & \#3 \\
\hline MYL12A & 2434375275 & 18 & 1 & 41 & $\mathrm{~A}$ & $<\mathrm{T}$ & NS & D & $\# 3$ \\
\hline AMAC1L1 & 2442732047 & 18 & 1 & 22 & $\mathrm{G}$ & $<\mathrm{A}$ & $\mathrm{S}$ & $\mathrm{N}$ & $\# 2$ \\
\hline C18orf1 & 2444767403 & 18 & 5 & 65 & $\mathrm{~A}$ & $<\mathrm{G}$ & $\mathrm{S}$ & D & $\# 3$ \\
\hline LOC 729774 & 2445483471 & 18 & 2 & 38 & $\mathrm{G}$ & $<\mathrm{T}$ & NS & NR & \#3 \\
\hline POTEC & 2445664872 & 18 & 1 & 68 & $\mathrm{~T}$ & $<\mathrm{C}$ & NS & $\mathrm{N}$ & $\# 2$ \\
\hline CTAGE1 & 2448017831 & 18 & 1 & 35 & $\mathrm{C}$ & $<\mathrm{T}$ & NS & $\mathrm{N}$ & $\# 1$ \\
\hline KCTD1 & 2452149054 & 18 & 1 & 18 & $\mathrm{~T}$ & $<\mathrm{G}$ & NS & $\mathrm{N}$ & $\# 3$ \\
\hline DSG4 & 2457014977 & 18 & 15 & 41 & $\mathrm{G}$ & $<\mathrm{T}$ & NS & NO & $\# 3$ \\
\hline FAM59A & 2457889818 & 18 & 4 & 30 & G & $<7$ & $\mathrm{~S}$ & $\mathrm{~N}$ & \#3 \\
\hline FAM59A & 2457889821 & 18 & 4 & 29 & A & $<\mathrm{C}$ & NS & $\mathrm{D}$ & \#3 \\
\hline MOCOS & 2461870479 & 18 & 15 & 143 & $\mathrm{~T}$ & $<\mathrm{C}$ & NS & $\mathrm{N}$ & $\# 4$ \\
\hline SLC14A2 & 2471234230 & 18 & 4 & 39 & G & $<\mathrm{A}$ & NS & $\mathrm{N}$ & $\# 3$ \\
\hline KIAA1632 & 2471505962 & 18 & 25 & 17 & A & $<\mathrm{T}$ & NS & $\mathrm{N}$ & $\# 3$ \\
\hline KIAA1632 & 2471505963 & 18 & 25 & 17 & A & $<\mathrm{T}$ & NS & NO & \#3 \\
\hline FUSSEL18 & 2472796815 & 18 & 1 & 35 & A & $<\mathrm{T}$ & NS & $\mathrm{D}$ & $\# 1$ \\
\hline ZBTB7C & 2473578004 & 18 & 2 & 78 & $\mathrm{~T}$ & $<\mathrm{G}$ & NS & $\mathrm{D}$ & $\# 4$ \\
\hline ZBTB7C & 2473588900 & 18 & 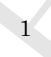 & 208 & $\mathrm{~T}$ & $<\mathrm{C}$ & $\mathrm{S}$ & $\mathrm{N}$ & $\# 4$ \\
\hline KIAA0427 & 2474259908 & 18 & 6 & 22 & $\mathrm{~T}$ & $<\mathrm{A}$ & NS & $\mathrm{N}$ & \#3 \\
\hline $\mathrm{CXXC1}$ & 2475832249 & 18 & 10 & 67 & A & $<\mathrm{G}$ & $\mathrm{S}$ & $\mathrm{N}$ & \#4 \\
\hline TCF4 & 2480799098 & 18 & 12 & 33 & $\mathrm{C}$ & $<\mathrm{A}$ & NS & $\mathrm{D}$ & $\# 3$ \\
\hline TCF4 & 2481003232 & 18 & 3 & 19 & $\mathrm{C}$ & $<\mathrm{A}$ & $\mathrm{S}$ & $\mathrm{N}$ & \#3 \\
\hline CCBE1 & 2485008619 & 18 & 4 & 99 & $\mathrm{C}$ & $<\mathrm{T}$ & $\mathrm{S}$ & $\mathrm{N}$ & $\# 2$ \\
\hline NETO1* & 2498404004 & 18 & 3 & 115 & $\mathrm{~A}$ & $<\mathrm{T}$ & NS & $\mathrm{D}$ & $\# 1$ \\
\hline C19orf6 & 2506845912 & 19 & 4 & 13 & $\mathrm{~T}$ & $<\mathrm{G}$ & $\mathrm{NS}$ & $\mathrm{D}$ & $\# 1$ \\
\hline $\mathrm{ABCA} 7$ & 2506887468 & 19 & 25 & 15 & A & $<\mathrm{G}$ & $\mathrm{S}$ & $\mathrm{N}$ & \#4 \\
\hline REXO1 & 2507659336 & 19 & 3 & 14 & $\mathrm{C}$ & $<\mathrm{T}$ & $\mathrm{S}$ & $\mathrm{N}$ & $\# 4$ \\
\hline FAM108A1 & 2507714358 & 19 & 2 & 13 & $\mathrm{~T}$ & $<\mathrm{C}$ & NS & $\mathrm{N}$ & \#4 \\
\hline PIP5K1C & 2509476658 & 19 & 13 & 27 & G & $<\mathrm{A}$ & NS & $\mathrm{N}$ & $\# 3$ \\
\hline MAP2K2 & 2509934448 & 19 & 6 & 47 & $\mathrm{C}$ & $<\mathrm{T}$ & NS & $\mathrm{D}$ & $\# 4$ \\
\hline ACER1 & 2512145698 & 19 & 3 & 26 & $\mathrm{~T}$ & $<\mathrm{C}$ & NS & $\mathrm{N}$ & $\# 4$ \\
\hline LASS4 & 2514105354 & 19 & 7 & 55 & $\mathrm{~T}$ & $<\mathrm{C}$ & $\mathrm{S}$ & $\mathrm{N}$ & \#4 \\
\hline
\end{tabular}




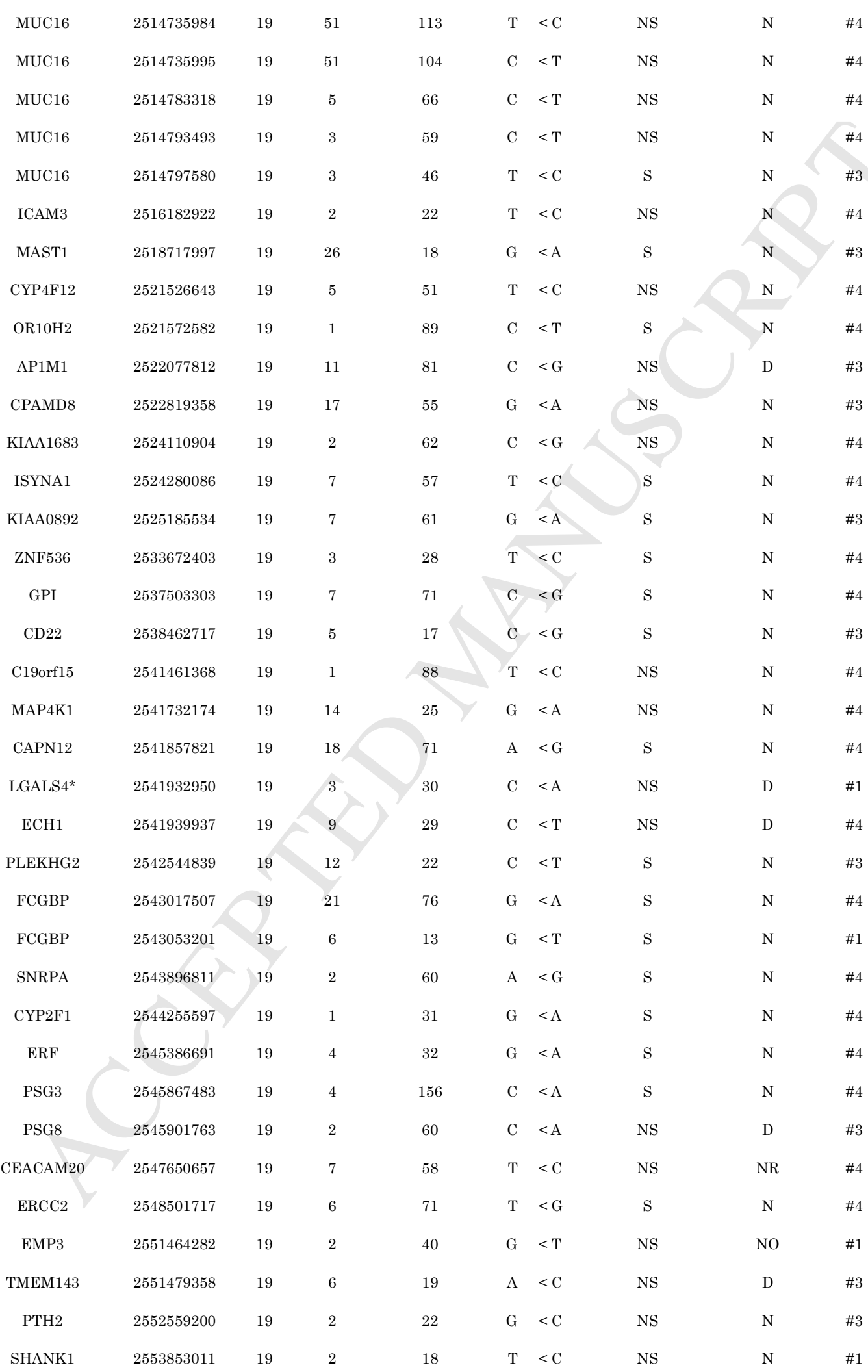




\begin{tabular}{|c|c|c|c|c|c|c|c|c|c|}
\hline ZNF808* & 2555691982 & 19 & 3 & 39 & G & $<\mathrm{A}$ & NS & $\mathrm{N}$ & \#1 \\
\hline ZNF765 & 2556544681 & 19 & 3 & 19 & $\mathrm{C}$ & $<\mathrm{G}$ & NS & $\mathrm{N}$ & $\# 1$ \\
\hline ZNF765 & 2556544684 & 19 & 3 & 19 & $\mathrm{~T}$ & $<\mathrm{C}$ & $\mathrm{S}$ & $\mathrm{N}$ & $\# 1$ \\
\hline ZNF761 & 2556586232 & 19 & 2 & 16 & $\mathrm{G}$ & $<\mathrm{A}$ & $\mathrm{S}$ & NR & $\# 4$ \\
\hline LILRB3* & 2557359732 & 19 & 3 & 208 & G & $<\mathrm{C}$ & NS & $\mathrm{N}$ & $\# 2$ \\
\hline LILRA1 & 2557740721 & 19 & 5 & 61 & $\mathrm{~T}$ & $<\mathrm{C}$ & NS & $\mathrm{N}$ & $\# 2$ \\
\hline KIR2DL4 & 2557949666 & 19 & 3 & 17 & $\mathrm{C}$ & $<\mathrm{G}$ & NS & $\mathrm{N}$ & $\# 3$ \\
\hline KIR3DL1 & 2557963191 & 19 & 3 & 109 & A & $<\mathrm{G}$ & $\mathrm{S}$ & $\mathrm{N}$ & $\# 4$ \\
\hline KIR2DS4 & 2557982650 & 19 & 3 & 37 & $\mathrm{~T}$ & $<\mathrm{G}$ & $\mathrm{S}$ & NR & $\# 2$ \\
\hline KIR2DS4 & 2557982701 & 19 & 3 & 36 & G & $<\mathrm{T}$ & NS & NR & $\# 2$ \\
\hline KIR2DS4 & 2557982728 & 19 & 3 & 21 & G & $<\mathrm{C}$ & NS & NR & $\# 2$ \\
\hline RDH13 & 2558201492 & 19 & 1 & 28 & $\mathrm{C}$ & $<\mathrm{T}$ & NS & $\mathrm{N}$ & $\# 4$ \\
\hline $\mathrm{RDH} 13$ & 2558201493 & 19 & 1 & 29 & G & & NS & $\mathrm{N}$ & $\# 4$ \\
\hline ZFP28 & 2559692350 & 19 & 3 & 33 & $\mathrm{C}$ & $<$ & $\mathrm{S}$ & $\mathrm{N}$ & $\# 3$ \\
\hline ZNF550* & 2560701027 & 19 & 1 & 67 & $\mathrm{C}$ & $<\mathrm{T}$ & NS & $\mathrm{D}$ & $\# 1$ \\
\hline ZSCAN22 & 2561483213 & 19 & 2 & 17 & $\mathrm{~T}$ & $<\mathrm{G}$ & NS & $\mathrm{N}$ & $\# 3$ \\
\hline KIR2DS1 & 2561871082 & 19 & 3 & 153 & A & $<\mathrm{G}$ & $\mathrm{S}$ & NR & $\# 2$ \\
\hline SIGLEC1 & 2565630685 & 20 & 3 & 19 & C & $<\mathrm{T}$ & NS & $\mathrm{N}$ & $\# 4$ \\
\hline PAK7 & 2571487871 & 20 & 4 & 131 & $\mathrm{C}$ & $<\mathrm{T}$ & NS & $\mathrm{N}$ & $\# 4$ \\
\hline FLRT3 & 2576252200 & 20 & 1 & 17 & $\mathrm{~T}$ & $<\mathrm{G}$ & NS & $\mathrm{D}$ & $\# 3$ \\
\hline CST9L & 2585490888 & 20 & 2 & 216 & $\mathrm{~T}$ & $<\mathrm{G}$ & NS & $\mathrm{N}$ & $\# 4$ \\
\hline BPI & 2595650275 & 20 & 11 & 148 & A & $<\mathrm{G}$ & NS & $\mathrm{N}$ & \#4 \\
\hline LBP & 2595672219 & 20 & 2 & 23 & G & $<\mathrm{A}$ & $\mathrm{S}$ & $\mathrm{N}$ & \#4 \\
\hline LBP* & 2595691968 & 20 & 10 & 25 & G & $<\mathrm{T}$ & $\mathrm{S}$ & $\mathrm{N}$ & $\# 1$ \\
\hline KIAA1219 & 2595847726 & 20 & 10 & 14 & $\mathrm{~T}$ & $<\mathrm{G}$ & NS & $\mathrm{D}$ & $\# 3$ \\
\hline PTPRT & 2599404902 & 20 & 31 & 37 & C & $<\mathrm{A}$ & NS & $\mathrm{D}$ & $\# 3$ \\
\hline SEMG2 & 2602544774 & 20 & 2 & 42 & A & $<\mathrm{G}$ & $\mathrm{S}$ & $\mathrm{N}$ & $\# 4$ \\
\hline ZNF335 & 2603273210 & 20 & 21 & 32 & A & $<\mathrm{G}$ & $\mathrm{S}$ & $\mathrm{N}$ & $\# 4$ \\
\hline PCK1 & 2614832083 & 20 & 3 & 52 & A & $<\mathrm{G}$ & $\mathrm{S}$ & $\mathrm{N}$ & $\# 4$ \\
\hline CTSZ & 2616266012 & 20 & 5 & 51 & A & $<\mathrm{G}$ & $\mathrm{S}$ & $\mathrm{N}$ & \#4 \\
\hline OGFR & 2620085220 & 20 & 4 & 20 & G & $<\mathrm{A}$ & $\mathrm{S}$ & $\mathrm{N}$ & $\# 4$ \\
\hline $\mathrm{KCNQ2}$ & 2620689776 & 20 & 14 & 14 & $\mathrm{C}$ & $<\mathrm{G}$ & NS & $\mathrm{N}$ & $\# 4$ \\
\hline LOC100132288 & 2622007697 & 21 & 2 & 24 & $\mathrm{C}$ & $<\mathrm{T}$ & NS & $\mathrm{NR}$ & $\# 4$ \\
\hline LOC100288017 & 2623267208 & 21 & 1 & 18 & G & $<\mathrm{A}$ & NS & NR & $\# 2$ \\
\hline POTED & 2623681322 & 21 & 1 & 60 & G & $<\mathrm{A}$ & NS & $\mathrm{N}$ & $\# 2$ \\
\hline KRTAP13-2 & 2640442822 & 21 & 1 & 81 & A & $<\mathrm{T}$ & NS & D & \#3 \\
\hline
\end{tabular}




\begin{tabular}{|c|c|c|c|c|c|c|c|c|c|}
\hline C21orf66 & 2642816546 & 21 & 12 & 14 & A & $<\mathrm{C}$ & $\mathrm{NS}$ & $\mathrm{N}$ & $\# 1$ \\
\hline C21orf66 & 2642816547 & 21 & 12 & 14 & C & $<\mathrm{T}$ & NS & $\mathrm{N}$ & $\# 1$ \\
\hline WRB & 2649461227 & 21 & 2 & 73 & G & $<\mathrm{T}$ & $\mathrm{NS}$ & NO & $\# 4$ \\
\hline WRB & 2649461228 & 21 & 2 & 73 & A & $<\mathrm{T}$ & $\mathrm{NS}$ & $\mathrm{D}$ & $\# 4$ \\
\hline DSCAM & 2650145634 & 21 & 27 & 116 & G & $<\mathrm{C}$ & $\mathrm{S}$ & $\mathrm{N}$ & $\# 4$ \\
\hline PRDM15 & 2651870131 & 21 & 31 & 65 & $\mathrm{G}$ & $<\mathrm{A}$ & $\mathrm{S}$ & $\mathrm{N}$ & $\# 4$ \\
\hline PFKL & 2654380692 & 21 & 4 & 19 & $\mathrm{C}$ & $<\mathrm{T}$ & $\mathrm{S}$ & $\mathrm{N}$ & $\# 4$ \\
\hline KRTAP10-6 & 2654660390 & 21 & 1 & 137 & $\mathrm{G}$ & $<\mathrm{A}$ & $\mathrm{S}$ & $\mathrm{N}$ & $\# 4$ \\
\hline KRTAP12-2 & 2654734983 & 21 & 1 & 59 & $\mathrm{C}$ & $<\mathrm{T}$ & $\mathrm{NS}$ & $\mathrm{N}$ & $\# 4$ \\
\hline KRTAP12-2 & 2654735333 & 21 & 1 & 71 & $\mathrm{G}$ & $<\mathrm{A}$ & $\mathrm{S}$ & $\mathrm{N}$ & $\# 4$ \\
\hline KRTAP12-2 & 2654735334 & 21 & 1 & 69 & $\mathrm{C}$ & $<\mathrm{T}$ & NS & $\mathrm{N}$ & $\# 4$ \\
\hline COL6A2 & 2656200935 & 21 & 27 & 63 & $\mathrm{C}$ & $<\mathrm{G}$ & $\mathrm{S}$ & $\mathrm{N}$ & $\# 3$ \\
\hline FTCD & 2656222648 & 21 & 2 & 55 & $\mathrm{~T}$ & $<$ & NS & $\mathrm{D}$ & $\# 2$ \\
\hline CECR5 & 2658218162 & 22 & 6 & 14 & $\mathrm{~T}$ & $<\mathrm{C}$ & $\mathrm{NS}$ & $\mathrm{D}$ & $\# 3$ \\
\hline CECR2 & 2658624712 & 22 & 16 & 25 & $\mathrm{C}$ & $<\mathrm{T}$ & $\mathrm{s}$ & $\mathrm{N}$ & $\# 4$ \\
\hline LOC100288065 & 2658662354 & 22 & 4 & 63 & A & $<\mathrm{G}$ & NS & $\mathrm{N}$ & $\# 4$ \\
\hline TBX1 & 2660347982 & 22 & 4 & 51 & C & $<\mathrm{T}$ & $\mathrm{S}$ & $\mathrm{N}$ & $\# 4$ \\
\hline ZNF280B* & 2663338671 & 22 & 1 & 78 & G & $<\mathrm{A}$ & $\mathrm{S}$ & $\mathrm{N}$ & $\# 2$ \\
\hline C22orf30 & 2672604693 & 22 & 3 & 17 & $\mathrm{C}$ & $<\mathrm{T}$ & NS & NO & $\# 3$ \\
\hline ISX & 2675974809 & 22 & 2 & 60 & G & $<\mathrm{A}$ & $\mathrm{S}$ & $\mathrm{N}$ & $\# 1$ \\
\hline HMGXB4 & 2676157665 & 22 & & 42 & A & $<\mathrm{T}$ & $\mathrm{S}$ & $\mathrm{N}$ & $\# 3$ \\
\hline APOL1 & 2677147183 & 22 & & 27 & $\mathrm{C}$ & $<\mathrm{T}$ & $\mathrm{NS}$ & $\mathrm{N}$ & $\# 1$ \\
\hline TMPRSS6 & 2677959079 & 22 & 17 & 144 & G & $<\mathrm{A}$ & $\mathrm{s}$ & $\mathrm{N}$ & $\# 4$ \\
\hline TMPRSS6 & 2677959089 & 22 & 17 & 133 & A & $<\mathrm{G}$ & $\mathrm{NS}$ & $\mathrm{N}$ & \#4 \\
\hline SSTR3 & 2678099174 & 22 & 1 & 18 & G & $<\mathrm{A}$ & $\mathrm{S}$ & $\mathrm{N}$ & $\# 4$ \\
\hline АРОВЕС3А & 2679853734 & 22 & 3 & 53 & $\mathrm{C}$ & $<\mathrm{T}$ & $\mathrm{S}$ & $\mathrm{N}$ & $\# 4$ \\
\hline L3MBTL2 & 2682109341 & 22 & 5 & 24 & $\mathrm{C}$ & $<\mathrm{T}$ & $\mathrm{S}$ & $\mathrm{N}$ & $\# 4$ \\
\hline NAGA & 2682959967 & 22 & 3 & 46 & $\mathrm{C}$ & $<\mathrm{T}$ & $\mathrm{s}$ & $\mathrm{N}$ & $\# 4$ \\
\hline TTLL12 & 2684071796 & 22 & 5 & 53 & C & $<\mathrm{T}$ & $\mathrm{s}$ & $\mathrm{N}$ & $\# 3$ \\
\hline SCUBE1 & 2684110469 & 22 & 15 & 48 & $\mathrm{C}$ & $<\mathrm{G}$ & $\mathrm{S}$ & $\mathrm{N}$ & $\# 4$ \\
\hline LOC100289317 & 2686220100 & 22 & 1 & 25 & $\mathrm{~A}$ & $<\mathrm{G}$ & NS & NR & $\# 4$ \\
\hline CELSR1 & 2687425845 & 22 & 1 & 58 & A & $<\mathrm{G}$ & NS & $\mathrm{N}$ & $\# 4$ \\
\hline MAPK8IP2 & 2691494874 & 22 & 11 & 52 & G & $<\mathrm{A}$ & NS & $\mathrm{N}$ & $\# 4$ \\
\hline CD99 & 2693971425 & $\mathrm{X}$ & 6 & 16 & G & $<\mathrm{A}$ & $\mathrm{NS}$ & $\mathrm{D}$ & $\# 4$ \\
\hline PRKX & 2694923444 & $\mathrm{x}$ & 2 & 88 & G & $<\mathrm{A}$ & $\mathrm{s}$ & $\mathrm{N}$ & $\# 4$ \\
\hline ARHGAP6* & 2702537703 & $\mathrm{x}$ & 4 & 81 & G & $<\mathrm{A}$ & NS & $\mathrm{D}$ & \#2 \\
\hline
\end{tabular}




\begin{tabular}{|c|c|c|c|c|c|c|c|c|c|}
\hline DMD & 2723711682 & $\mathrm{X}$ & 37 & 92 & $\mathrm{~T}$ & $<\mathrm{C}$ & NS & $\mathrm{N}$ & $\# 4$ \\
\hline WAS & 2739828502 & $\mathrm{X}$ & 11 & 13 & G & $<\mathrm{T}$ & NS & $\mathrm{N}$ & $\# 3$ \\
\hline GATA1 & 2739931290 & $\mathrm{X}$ & 2 & 14 & A & $<\mathrm{C}$ & NS & $\mathrm{N}$ & $\# 3$ \\
\hline GAGE12E & 2740547782 & $\mathrm{X}$ & 1 & 62 & G & $<\mathrm{T}$ & NS & $\mathrm{D}$ & $\# 4$ \\
\hline PAGE1* & 2740686656 & $\mathrm{X}$ & 3 & 37 & $\mathrm{~T}$ & $<\mathrm{C}$ & $\mathrm{S}$ & $\mathrm{N}$ & $\# 1$ \\
\hline USP27X & 2740875896 & $\mathrm{X}$ & 1 & 39 & $\mathrm{G}$ & $<\mathrm{C}$ & NS & NR & $\# 3$ \\
\hline TSPYL2 & 2744246047 & $\mathrm{X}$ & 6 & 76 & A & $<\mathrm{G}$ & NS & $\mathrm{N}$ & $\# 3$ \\
\hline FAM120C & 2745238526 & $\mathrm{X}$ & 14 & 66 & $\mathrm{C}$ & $<\mathrm{A}$ & NS & $\mathrm{D}$ & $\# 2$ \\
\hline ITIH5L & 2745914425 & $\mathrm{X}$ & 8 & 22 & G & $<\mathrm{A}$ & NS & $\mathrm{N}$ & $\# 3$ \\
\hline MSN & 2752987418 & $\mathrm{X}$ & 9 & 47 & $\mathrm{G}$ & $<\mathrm{A}$ & $\mathrm{S}$ & $\mathrm{N}$ & $\# 2$ \\
\hline OPHN1 & 2755314567 & $\mathrm{X}$ & 20 & 47 & $\mathrm{~T}$ & $<\mathrm{C}$ & NS & $\mathrm{N}$ & $\# 3$ \\
\hline DGAT2L6 & 2757452592 & $\mathrm{X}$ & 5 & 20 & $\mathrm{C}$ & $<\mathrm{T}$ & $\mathrm{S}$ & $\mathrm{N}$ & $\# 3$ \\
\hline LPAR4 & 2765991350 & $\mathrm{x}$ & 1 & 46 & G & $<$ & NS & $\mathrm{D}$ & $\# 3$ \\
\hline LPAR4 & 2765991352 & $\mathrm{x}$ & 1 & 43 & $\mathrm{C}$ & $<$ & $\mathrm{S}$ & $\mathrm{N}$ & \#3 \\
\hline PCDH11X & 2779854213 & $\mathrm{x}$ & 7 & 171 & $\mathrm{C}$ & $<\mathrm{A}$ & NS & $\mathrm{N}$ & $\# 3$ \\
\hline SYTL4 & 2787924130 & $\mathrm{x}$ & 9 & 24 & G & $<\mathrm{A}$ & $\mathrm{S}$ & $\mathrm{N}$ & $\# 3$ \\
\hline SYTL4 & 2787924131 & $\mathrm{x}$ & 9 & 24 & $\mathrm{~T}$ & $<\mathrm{C}$ & NS & $\mathrm{D}$ & $\# 3$ \\
\hline NXF5 & 2789077402 & $\mathrm{x}$ & 3 & 81 & $\mathrm{~T}$ & $<\mathrm{C}$ & NS & $\mathrm{D}$ & $\# 1$ \\
\hline NXF2 & 2789554971 & $\mathrm{x}$ & 10 & 19 & $\mathrm{C}$ & $<\mathrm{T}$ & $\mathrm{S}$ & $\mathrm{N}$ & $\# 3$ \\
\hline CLDN2 & 2794152808 & $\mathrm{x}$ & 1 & 26 & $\mathrm{C}$ & $<\mathrm{A}$ & NS & $\mathrm{N}$ & $\# 3$ \\
\hline CLDN2 & 2794152809 & $\mathrm{X}$ & 1 & 26 & $\mathrm{C}$ & $<\mathrm{T}$ & NS & $\mathrm{N}$ & $\# 3$ \\
\hline TRPC5 & 2799176319 & $\mathrm{x}$ & + & 24 & G & $<\mathrm{A}$ & NS & $\mathrm{D}$ & $\# 3$ \\
\hline TRPC5 & 2799176320 & $\mathrm{x}$ & 1 & 24 & G & $<\mathrm{T}$ & NS & $\mathrm{N}$ & $\# 3$ \\
\hline RHOXF2B & 2807087244 & $\mathrm{x}$ & 4 & 29 & A & $<\mathrm{G}$ & NS & $\mathrm{N}$ & $\# 3$ \\
\hline PLAC1 & 2821580905 & $\mathrm{X}$ & 1 & 20 & A & $<\mathrm{G}$ & NS & $\mathrm{N}$ & $\# 3$ \\
\hline RBMX & 2823837181 & $\mathrm{x}$ & 8 & 40 & G & $<\mathrm{C}$ & NS & $\mathrm{D}$ & $\# 4$ \\
\hline SLITRK4 & 2830598721 & $\mathrm{X}$ & 1 & 56 & $\mathrm{~T}$ & $<\mathrm{C}$ & NS & $\mathrm{N}$ & \#3 \\
\hline NSDHL & 2839816887 & $\mathrm{X}$ & 6 & 109 & $\mathrm{~A}$ & $<\mathrm{G}$ & NS & $\mathrm{D}$ & $\# 3$ \\
\hline MPP1 & 2841799005 & $\mathrm{X}$ & 5 & 36 & $\mathrm{~T}$ & $<\mathrm{C}$ & $\mathrm{S}$ & $\mathrm{N}$ & $\# 3$ \\
\hline MPP1 & 2841799006 & $\mathrm{X}$ & 5 & 36 & $\mathrm{~T}$ & $<\mathrm{C}$ & NS & $\mathrm{N}$ & $\# 3$ \\
\hline RBMY1D & 2863471281 & $\mathrm{Y}$ & 11 & 23 & $\mathrm{~T}$ & $<\mathrm{C}$ & $\mathrm{S}$ & $\mathrm{N}$ & $\# 1$ \\
\hline
\end{tabular}

*: These genes were commonly mutated in the synchronously developed HCCs of cases \#1 and \#2.

a: chromosome, b: coding sequence, $c$ : $\mathrm{N}$ : non-synonymous mutation, $\mathrm{S}$ : synonymous mutation, $\mathrm{d}$ : Functional predictions by SIFT. D; deleterious, N; neutral, NO; nonsense mutation, NR: no record found 
Supplemental Table 4. Functional relevance of mutations detected in the HCC tumors.

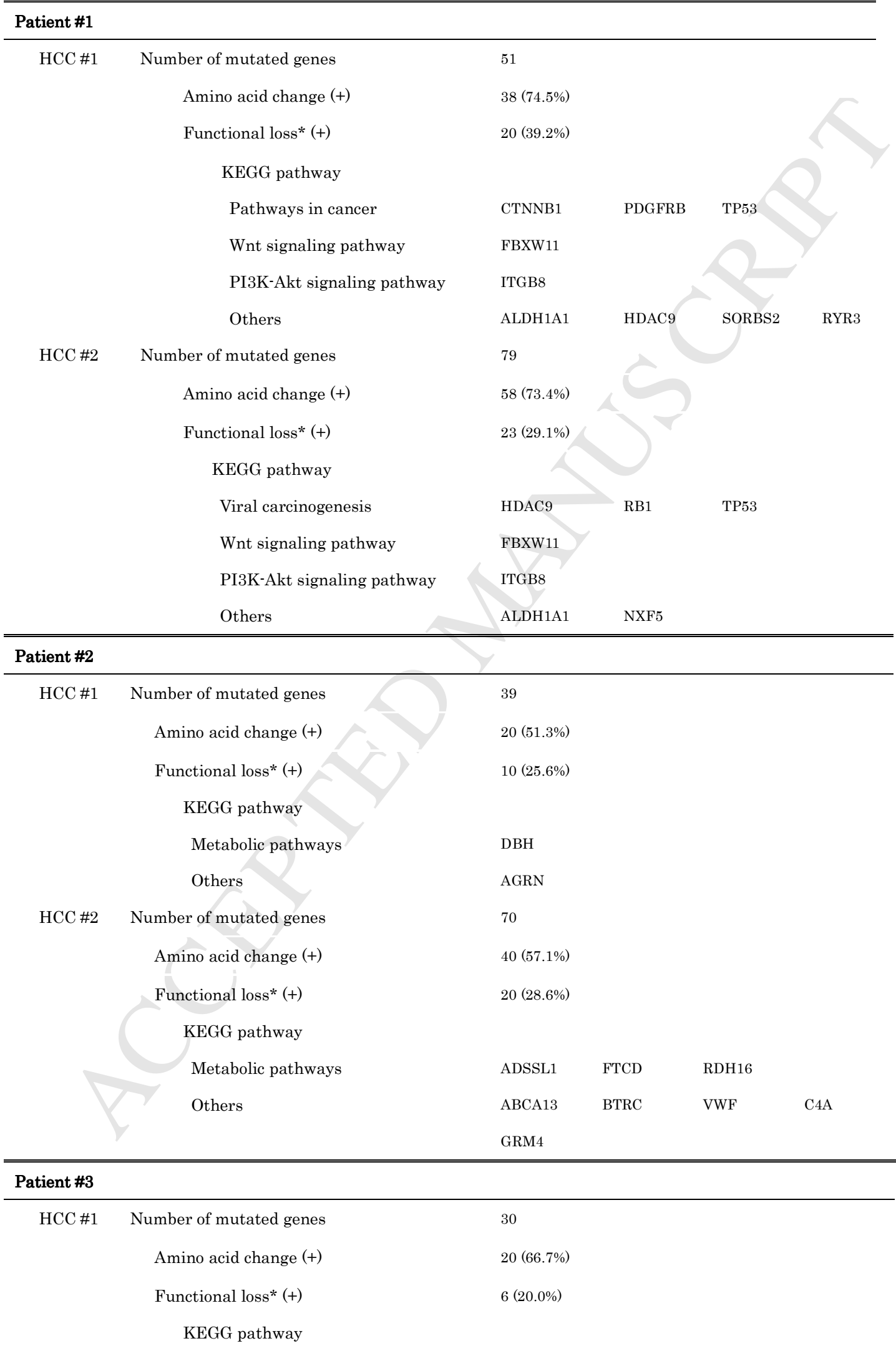


Functional loss* (+)

$90(32.6 \%)$

KEGG pathway

Metabolic pathways

ACSM4

UGT2B28

DHRS4L2

GALNT5

ME1

POLE

NSDHL

PIK3C2G

PI3K-Akt signaling pathway

COL6A6

HGF

ANGPT

LPAR4

Neuroactive ligand-receptor

GLRA2

Others

\begin{tabular}{llll} 
CDK9 & CA2 & ABCC12 & AP1M1 \\
GNPAT & GLYAT & RUVBL1 & GDF9 \\
MYL12A & MLL3 & SLC18A2 & MAP4K4 \\
PRPF8 & PIP4K2A & SLC9A4 & NUP37 \\
VCP & TTN & & \\
\hline
\end{tabular}

\title{
Patient \#4
}

$\mathrm{HCC}$

\author{
Number of mutated genes \\ Amino acid change (+) \\ Functional loss* $(+)$ \\ KEGG pathway
}

364

$177(48.6 \%)$

$46(12.6 \%)$

\begin{tabular}{llllll} 
Metabolic pathways & ACSM5 & ALPP & PNLIPRP1 & \\
MAPK signaling pathway & HSPA6 & MAP2K2 & & \\
PI3K-Akt signaling pathway & FLT4 & & & \\
Others & ECH1 & CHI3L1 & FURIN & CD99 \\
& KCNJ12 & ITGAE & TMPRSS4 & REXO1L1 \\
& RBMX & PLOD3 & TUBA4A & PGA3 \\
\hline
\end{tabular}

*The number of mutated genes predicted to be "damaging (deleterious)" by Sorting Intolerant From Tolerant (SIFT) functional impact predictions (http://provean.jcvi.org/index.php).

The genes categorized in multiple pathways are shown in only one representative pathway. 
Supplemental Table 5. List of 448 indels in 409 genes at a frequency of more than $20 \%$ of reads in $7 \mathrm{HCC}$ tumors of 4 cases.

\begin{tabular}{|c|c|c|c|c|c|c|}
\hline Reference Position & Gene & $\mathrm{Chr}^{\mathrm{a}}$ & $\mathrm{CDS}^{\mathrm{b}}$ & Coverage & Allele change & Case \\
\hline 7813482 & ERRFI1 & 1 & 3 & 17 & insA & $\# 3$ \\
\hline $12628605^{*}$ & PRAMEF11 & 1 & 3 & 27 & insC & $\# 2$ \\
\hline 12718307 & PRAMEF7 & 1 & 2 & 47 & insT & $\# 3$ \\
\hline $17358674^{*}$ & PADI6 & 1 & 9 & 41 & de & $\# 4$ \\
\hline $17358674^{*}$ & PADI6 & 1 & 9 & 62 & delGT & $\# 2$ \\
\hline 26696284 & ARID1A & 1 & 2 & 19 & delC & $\# 4$ \\
\hline $31395890^{*}$ & SERINC2 & 1 & 9 & 17 & insG & $\# 3$ \\
\hline 46770747 & CYP4B1 & 1 & 8 & 42 & delAT & $\# 4$ \\
\hline 46770748 & CYP4B1 & 1 & 8 & 42 & delT & $\# 4$ \\
\hline $52949277^{*}$ & LOC100133211 & 1 & 1 & 42 & delG & $\# 4$ \\
\hline 53189215 & MAGOH & 1 & 3 & 25 & insA & $\# 3$ \\
\hline $54095320^{*}$ & CDCP2 & 1 & 4 & 21 & insC & $\# 4$ \\
\hline 62557547 & ANGPTL3 & 1 & 4 & 17 & ins $\mathrm{T}$ & $\# 3$ \\
\hline 78876012 & ELTD1 & 1 & 10 & 13 & insA & $\# 3$ \\
\hline 89014597 & GBP1 & 1 & 4 & 17 & insA & $\# 2$ \\
\hline 90670304 & BARHL2 & 1 & 2 & 20 & insC & $\# 3$ \\
\hline 108974106 & CLCC1 & 1 & 6 & 84 & insG & $\# 4$ \\
\hline $122705624^{*}$ & E4DIP & 1 & 14 & 80 & delG & $\# 4$ \\
\hline $122713730^{*}$ & PDE4DIP & 1 & 6 & 465 & delT & $\# 1$ \\
\hline 131401399 & DENND4B & 1 & 12 & 43 & insG & $\# 3$ \\
\hline 131474740 & NUP210L & 1 & 34 & 13 & insG & $\# 3$ \\
\hline 131951749 & $\mathrm{SHF}$ & 1 & 3 & 112 & insA & $\# 2$ \\
\hline 133072970 & MSTO1 & 1 & 11 & 33 & insA & $\# 3$ \\
\hline 133795656 & ССT3 & 1 & 2 & 34 & ins $\mathrm{T}$ & $\# 3$ \\
\hline $133844355^{*}$ & RHBG & 1 & 9 & 40 & delC & $\# 4$ \\
\hline $134043234^{*}$ & TTC24 & 1 & 3 & 12 & delC & $\# 3$ \\
\hline 136505109 & IFI16 & 1 & 7 & 31 & insT & $\# 3$ \\
\hline 146999551 & F5 & 1 & 13 & 23 & insT & $\# 3$ \\
\hline 151907797 & GPR52 & 1 & 1 & 18 & insA & $\# 2$ \\
\hline 162596811 & C1orf25 & 1 & 10 & 26 & ins $\mathrm{T}$ & $\# 3$ \\
\hline 163815430 & TPR & 1 & 15 & 43 & delT & $\# 3$ \\
\hline 174374155 & CFHR4 & 1 & 5 & 18 & insC & $\# 3$ \\
\hline
\end{tabular}




\begin{tabular}{|c|c|c|c|c|c|c|}
\hline 177507300 & NR5A2 & 1 & 5 & 20 & insT & $\# 3$ \\
\hline 201685738 & NVL & 1 & 6 & 32 & insA & \#3 \\
\hline 203543660 & ACBD3 & 1 & 2 & 18 & insG & $\# 3$ \\
\hline $208315870^{*}$ & ARV1 & 1 & 3 & 146 & delCT & $\# 1$ \\
\hline 208315871* & ARV1 & 1 & 3 & 144 & delT & $\# 1$ \\
\hline 213162772 & LYST & 1 & 3 & 19 & insG & \#3 \\
\hline 214770350 & RYR2 & 1 & 11 & 22 & insG & \#3 \\
\hline 219641707 & PLD5 & 1 & 2 & 102 & insA & $\# 1$ \\
\hline 224245114 & AHCTF1 & 1 & 14 & 19 & insG & $\# 3$ \\
\hline 230467928 & RNASEH1 & 2 & 8 & 13 & ins T & $\# 3$ \\
\hline 236404886 & ADAM17 & 2 & 19 & 29 & insG & $\# 3$ \\
\hline $251086730^{*}$ & LOC375190 & 2 & 8 & 40 & ins $\mathrm{C}$ & $\# 4$ \\
\hline 254371933 & IFT172 & 2 & 38 & 18 & ins $\mathrm{T}$ & $\# 3$ \\
\hline $258505433^{*}$ & SRD5A2 & 2 & 1 & 28 & insG & $\# 2$ \\
\hline 267355164 & SLC8A1 & 2 & 1 & 15 & insG & $\# 3$ \\
\hline 282849120 & EFEMP1 & 2 & 1 & 14 & insA & $\# 3$ \\
\hline 287714203 & PAPOLG & 2 & 15 & 20 & delT & \#3 \\
\hline 288274645 & USP34 & 2 & 15 & 14 & ins $\mathrm{C}$ & \#3 \\
\hline 301408194 & CCDC142 & 2 & 2 & 27 & insC & $\# 3$ \\
\hline 302628924 & C2orf3 & 2 & 3 & 22 & insA & $\# 3$ \\
\hline 312276713 & RETSAT & 2 & 4 & 55 & delC & \#2 \\
\hline 314781292 & RGPD2 & 2 & 5 & 27 & ins $\mathrm{T}$ & $\# 3$ \\
\hline $317672121^{*}$ & LOC391405 & 2 & 4 & 43 & delA & $\# 4$ \\
\hline 318709414 & TRIM43 & 2 & 1 & 22 & insA & $\# 3$ \\
\hline 325574912 & SLC9A4 & 2 & 6 & 27 & inst & $\# 3$ \\
\hline 329490991 & RGPD3 & 2 & 20 & 29 & insT & $\# 3$ \\
\hline 331565693 & $\mathrm{GCC} 2$ & 2 & 22 & 18 & insC & $\# 2$ \\
\hline 332910299 & RGPD5 & 2 & 21 & 25 & insA & $\# 3$ \\
\hline 333616906 & RGPD7 & 2 & 8 & 21 & delA & $\# 3$ \\
\hline 335725611 & SLC20A1 & 2 & 8 & 14 & insC & \#3 \\
\hline 336700192 & RABL2A & 2 & 4 & 76 & delG & $\# 1$ \\
\hline 340887331 & DDX18 & 2 & 7 & 18 & inst & $\# 3$ \\
\hline 350555029 & IWS1 & 2 & 11 & 62 & delG & $\# 3$ \\
\hline $355383031^{*}$ & ZNF806 & 2 & 3 & 50 & delC & $\# 4$ \\
\hline $355383457^{*}$ & ZNF806 & 2 & 3 & 56 & insA & \#4 \\
\hline $355383669^{*}$ & ZNF806 & 2 & 3 & 52 & delA & \#4 \\
\hline
\end{tabular}




\begin{tabular}{|c|c|c|c|c|c|c|}
\hline 361736116 & NXPH2 & 2 & 2 & 48 & insT & \#3 \\
\hline 385464478 & KCNH7 & 2 & 10 & 41 & insT & $\# 3$ \\
\hline 387807820 & COBLL1 & 2 & 2 & 23 & insA & $\# 3$ \\
\hline 388452944 & $\mathrm{SCN} 2 \mathrm{~A}$ & 2 & 26 & 57 & insT & $\# 3$ \\
\hline 400689016 & TTC30A & 2 & 1 & 32 & insA & \#3 \\
\hline 401650351 & TTN & 2 & 270 & 19 & insG & \#3 \\
\hline 401670059 & TTN & 2 & 242 & 25 & insT & \#3 \\
\hline 401800064 & TTN & 2 & 64 & 26 & insA & \#3 \\
\hline 402189048 & SESTD1 & 2 & 14 & 31 & insG & $\# 3$ \\
\hline 403038177 & CWC22 & 2 & 11 & 16 & insA & \#3 \\
\hline 418958541 & DNAH7 & 2 & 34 & 27 & ins $\mathrm{C}$ & $\# 3$ \\
\hline 439213578 & XRCC5 & 2 & 13 & 22 & delC & \#3 \\
\hline 446013793 & ACSL3 & 2 & 14 & 26 & ins $\mathrm{T}$ & $\# 3$ \\
\hline 446670417 & SCG2 & 2 & 1 & 17 & insA & $\# 3$ \\
\hline 446671148 & SCG2 & 2 & 1 & 30 & insC & $\# 3$ \\
\hline $456401277^{*}$ & SAG & 2 & 10 & 76 & delA & $\# 2$ \\
\hline 463724350 & $\mathrm{AQP} 12 \mathrm{~B}$ & 2 & 1 & 26 & delC & $\# 2$ \\
\hline $479793553^{*}$ & GRIP2 & 3 & 10 & 55 & insG & $\# 4$ \\
\hline 504388108 & TTC21A & 3 & 6 & 13 & insA & \#3 \\
\hline 509772719 & ZNF852 & 3 & 3 & 20 & delTC & $\# 4$ \\
\hline 509772720 & ZNF852 & 3 & 3 & 19 & delC & \#4 \\
\hline 511646368 & CCR5 & 3 & 1 & 24 & insT & $\# 3$ \\
\hline $515483759^{*}$ & SLC38A3 & 3 & 2 & 18 & insG & \#4 \\
\hline 538148646 & GLT8D4 & 3 & 6 & 14 & insC & \#3 \\
\hline $538243406^{*}$ & FLJ10213 & 3 & 1 & 12 & insA & $\# 2$ \\
\hline 538564462 & PDZRN3 & 3 & 10 & 24 & insG & $\# 3$ \\
\hline $540846731^{*}$ & LOC100288801 & 3 & 2 & 39 & delG & $\# 2$ \\
\hline 540918687 & ZNF717 & 3 & 4 & 18 & delC & $\# 1$ \\
\hline 570208682 & HHLA2 & 3 & 4 & 18 & insT & $\# 3$ \\
\hline 570487430 & DZIP3 & 3 & 10 & 45 & insT & $\# 3$ \\
\hline 574780156 & CD200R1 & 3 & 4 & 24 & insA & $\# 3$ \\
\hline 587083495 & ZNF148 & 3 & 6 & 14 & insG & $\# 3$ \\
\hline $591946859^{*}$ & LOC644974 & 3 & 6 & 36 & delC & \#3 \\
\hline 595459401 & TOPBP1 & 3 & 26 & 16 & insT & $\# 3$ \\
\hline 608303870 & PLSCR2 & 3 & 4 & 59 & insG & \#3 \\
\hline 611616867 & C3orf16 & 3 & 5 & 22 & delCT & \#3 \\
\hline
\end{tabular}




\begin{tabular}{|c|c|c|c|c|c|c|}
\hline 611616868 & C3orf16 & 3 & 5 & 21 & delT & $\# 3$ \\
\hline 612474548 & SELT & 3 & 4 & 38 & inst & $\# 4$ \\
\hline 631967149 & PHC3 & 3 & 10 & 13 & inst & $\# 3$ \\
\hline 648156647 & DGKG & 3 & 2 & 44 & insC & $\# 3$ \\
\hline 652237997* & CLDN16 & 3 & 1 & 271 & delG & \#2 \\
\hline 658673909 & PAK2 & 3 & 12 & 16 & inst & \#3 \\
\hline 660166378 & ZNF595 & 4 & 4 & 17 & insA & $\# 1$ \\
\hline $662098266^{*}$ & POLN & 4 & 23 & 65 & delG & \#3 \\
\hline 696277331 & FLJ16686 & 4 & 3 & 53 & delC & $\# 1$ \\
\hline 725715810 & TMPRSS11F & 4 & 7 & 30 & insA & $\# 3$ \\
\hline 728128121 & LOC100129410 & 4 & 3 & 13 & insC & $\# 2$ \\
\hline 752860649 & UNC5C & 4 & 14 & 17 & inst & \#3 \\
\hline $767688857^{*}$ & EGF & 4 & 24 & 16 & insC & $\# 1$ \\
\hline 779934903 & KIAA1109 & 4 & 39 & 17 & inst & $\# 3$ \\
\hline 782346647 & ANKRD50 & 4 & 3 & 37 & inst & $\# 3$ \\
\hline 827268418 & NEK1 & 4 & 4 & 17 & inst $\mathrm{T}$ & $\# 3$ \\
\hline 841124368 & CDKN2AIP & 4 & 3 & 16 & insA & $\# 3$ \\
\hline 853638502 & KIAA0947 & 5 & 14 & 24 & inst & $\# 3$ \\
\hline $889026155^{*}$ & CARD6 & 5 & 3 & 32 & inst & $\# 2$ \\
\hline 891727995 & PAIP1 & 5 & 2 & 16 & insG & $\# 2$ \\
\hline 901348948 & MAP3K1 & 5 & 13 & 24 & insC & $\# 3$ \\
\hline 909928153 & ADAMTS6 & 5 & 3 & 23 & insG & $\# 3$ \\
\hline 914509435 & SERF1B & 5 & 3 & 15 & insG & $\# 3$ \\
\hline 914509482 & SERF1B & 5 & 3 & 49 & insA & $\# 3$ \\
\hline $915509411^{*}$ & GTF2H2 & 5 & 13 & 31 & inst & $\# 2$ \\
\hline 919186632 & HEXB & 5 & 11 & 43 & insA & $\# 3$ \\
\hline 922917852 & SCAMP1 & 5 & 7 & 37 & insA & $\# 1$ \\
\hline 928534269 & EDIL3 & 5 & 7 & 25 & insA & \#3 \\
\hline 931867232 & $\mathrm{CCNH}$ & 5 & 7 & 26 & inst & $\# 3$ \\
\hline 956697390 & EPB41L4A & 5 & 11 & 13 & inst $\mathrm{T}$ & $\# 1$ \\
\hline 966610635 & ZNF474 & 5 & 1 & 39 & delT & $\# 1$ \\
\hline 972596345 & SLC12A2 & 5 & 8 & 35 & ins $\mathrm{T}$ & $\# 3$ \\
\hline 980635083* & SMAD5 & 5 & 6 & 105 & insC & $\# 1$ \\
\hline $985314450^{*}$ & LOC100288105 & 5 & 1 & 14 & delC & $\# 4$ \\
\hline $985640033^{*}$ & PCDHB9 & 5 & 1 & 32 & insA & $\# 1$ \\
\hline 985844899* & PCDHGA8 & 5 & 1 & 27 & delC & $\# 3$ \\
\hline
\end{tabular}




\begin{tabular}{|c|c|c|c|c|c|c|}
\hline 992330379 & SCGB3A2 & 5 & 1 & 14 & delA & $\# 3$ \\
\hline $994446878^{*}$ & TIGD6 & 5 & 1 & 136 & delT & \#1 \\
\hline 994476149 & HMGXB3 & 5 & 6 & 14 & delA & $\# 3$ \\
\hline 998157358 & GRIA1 & 5 & 11 & 19 & ins $\mathrm{C}$ & $\# 3$ \\
\hline 1020539380 & FAM153B & 5 & 4 & 23 & insC & \#3 \\
\hline 1039337531 & C6orf114 & 6 & 1 & 30 & insA & \#3 \\
\hline 1052252527 & BTN2A2 & 6 & 2 & 44 & insG & \#3 \\
\hline $1054107191^{*}$ & ZNF187 & 6 & 1 & 33 & insG & $\# 4$ \\
\hline $1056096293^{*}$ & FLJ45422 & 6 & 2 & 18 & insT & $\# 2$ \\
\hline $1057247419^{*}$ & MICA & 6 & 5 & 27 & delG & $\# 3$ \\
\hline 1082305754 & DST & 6 & 45 & 18 & insT & $\# 3$ \\
\hline 1088830738 & EYS & 6 & 6 & 19 & insT & $\# 3$ \\
\hline 1093406718 & COL19A1 & 6 & 5 & 1 & insA & $\# 3$ \\
\hline 1113248546 & MDN1 & 6 & 15 & 14 & ins $\mathrm{C}$ & $\# 3$ \\
\hline 1113280524 & MDN1 & 6 & 2 & 40 & insA & $\# 3$ \\
\hline $1131602437^{*}$ & FOXO3 & 6 & 2 & 64 & insG & $\# 3$ \\
\hline 1133380782 & SLC22A16 & 6 & 4 & 20 & insA & $\# 3$ \\
\hline 1135037748 & C6orf225 & 6 & 1 & 17 & delC & $\# 3$ \\
\hline 1153093327 & SAMD3 & 6 & 7 & 14 & delC & $\# 3$ \\
\hline 1154647636 & LOC643854 & 6 & 1 & 26 & insT & $\# 3$ \\
\hline 1154648098 & LOC643854 & 6 & 1 & 20 & insC & $\# 3$ \\
\hline 1159216432 & BCLAF1 & 6 & 2 & 13 & delT & $\# 2$ \\
\hline 1161156444 & PBOV1 & 6 & 1 & 36 & insG & $\# 3$ \\
\hline 1182019086 & RSPH3 & 6 & 6 & 43 & insA & $\# 3$ \\
\hline 1200500350 & RSPH10B2 & 7 & 19 & 23 & insG & $\# 3$ \\
\hline 1206053594 & VWDE & 7 & 19 & 26 & insA & $\# 4$ \\
\hline 1221518316 & TAX1BP1 & 7 & 13 & 14 & insA & $\# 3$ \\
\hline $1222659823^{*}$ & KIAA0644 & 7 & 1 & 90 & delC & $\# 4$ \\
\hline $1222659922^{*}$ & KIAA0644 & 7 & 1 & 26 & insC & $\# 3$ \\
\hline 1226974977 & BBS9 & 7 & 7 & 19 & inst & $\# 1$ \\
\hline $1228643735^{*}$ & DPY19L1 & 7 & 18 & 22 & delAT & $\# 4$ \\
\hline $1228643736^{*}$ & DPY19L1 & 7 & 18 & 50 & delT & $\# 1$ \\
\hline 1262731853 & TYW1B & 7 & 8 & 142 & delA & $\# 4$ \\
\hline 1262954278 & TRIM74 & 7 & 2 & 24 & insA & $\# 2$ \\
\hline 1265555276 & TRIM73 & 7 & 2 & 84 & insT & $\# 2$ \\
\hline $1266437261^{*}$ & FLJ37078 & 7 & 14 & 43 & insC & \#2 \\
\hline
\end{tabular}




\begin{tabular}{|c|c|c|c|c|c|c|}
\hline $1266593512^{*}$ & $\mathrm{ZP} 3$ & 7 & 8 & 51 & insG & $\# 1$ \\
\hline $1266763110^{*}$ & POMZP3 & 7 & 5 & 83 & delA & $\# 4$ \\
\hline 1278946055 & C7orf62 & 7 & 1 & 20 & insC & $\# 3$ \\
\hline 1283360469 & HEPACAM2 & 7 & 4 & 29 & inst & \#3 \\
\hline 1283589759 & CALCR & 7 & 9 & 26 & inst & $\# 3$ \\
\hline $1290893801^{*}$ & ZAN & 7 & 30 & 28 & insG & $\# 3$ \\
\hline 1291366094 & MOGAT3 & 7 & 2 & 24 & insA & $\# 3$ \\
\hline $1291722996^{*}$ & EMID2 & 7 & 13 & 20 & insG & $\# 4$ \\
\hline 1292538685 & LOC100289561 & 7 & 1 & 14 & insA & $\# 3$ \\
\hline 1295252926 & MLL5 & 7 & 12 & 21 & insG & $\# 3$ \\
\hline 1298402792 & NRCAM & 7 & 1 & 17 & ins $\mathrm{T}$ & $\# 3$ \\
\hline $1319055841^{*}$ & $\mathrm{KCP}$ & 7 & 10 & 62 & ins $\mathrm{C}$ & $\# 1$ \\
\hline 1319073009 & $\mathrm{KCP}$ & 7 & 1 & 30 & delC & $\# 2$ \\
\hline 1333766379 & LOC441294 & 7 & 1 & 46 & insA & $\# 4$ \\
\hline 1334380185 & CTAGE4 & 7 & 1 & 39 & insA & $\# 3$ \\
\hline 1334381975 & ARHGEF5L & 7 & 1 & 19 & insA & $\# 1$ \\
\hline $1339923632^{*}$ & KRBA1 & 7 & 12 & 76 & insC & $\# 2$ \\
\hline $1339973995^{*}$ & SSPO & 7 & 9 & 44 & $\mathrm{insC}$ & $\# 1$ \\
\hline $1340003537^{*}$ & SSPO & 7 & 60 & 15 & insC & $\# 4$ \\
\hline 1340012514 & SSPO & 7 & 76 & 23 & delA & $\# 2$ \\
\hline 1340015859 & SSPO & 7 & 83 & 14 & delC & $\# 2$ \\
\hline 1340525483 & C7orf29 & 7 & 1 & 24 & delC & $\# 1$ \\
\hline $1341211228^{*}$ & ATG9B & 7 & 10 & 49 & insC & $\# 1$ \\
\hline 1341434558 & SMARCD3 & 7 & 10 & 21 & delC & $\# 3$ \\
\hline 1342197228 & GALNTL5 & 7 & 5 & 71 & delT & $\# 4$ \\
\hline $1342442397^{*}$ & MLL3 & 7 & 14 & 208 & ins $\mathrm{T}$ & $\# 4$ \\
\hline $1356372261^{*}$ & XKR5 & 8 & 6 & 55 & delAG & $\# 1$ \\
\hline $1374409954^{*}$ & NEFL & 8 & 3 & 38 & delG & $\# 4$ \\
\hline $1380219728^{*}$ & UBXN8 & 8 & 7 & 83 & inst & $\# 1$ \\
\hline 1380304215 & TEX15 & 8 & 1 & 23 & insA & $\# 3$ \\
\hline $1388426070^{*}$ & PLEKHA2 & 8 & 11 & 28 & delC & $\# 2$ \\
\hline $1395399601^{*}$ & PRKDC & 8 & 31 & 17 & insG & $\# 1$ \\
\hline 1398930064 & PXDNL & 8 & 14 & 27 & insA & $\# 3$ \\
\hline $1410692513^{*}$ & YTHDF3 & 8 & 4 & 24 & insG & $\# 1$ \\
\hline 1415952398 & C8orf34 & 8 & 2 & 32 & insG & $\# 3$ \\
\hline 1445261384 & LAPTM4B & 8 & 2 & 16 & insC & \#3 \\
\hline
\end{tabular}




\begin{tabular}{|c|c|c|c|c|c|c|}
\hline $1490189877^{*}$ & JRK & 8 & 1 & 12 & delCA & $\# 3$ \\
\hline $1490189878^{*}$ & JRK & 8 & 1 & 19 & delA & \#2 \\
\hline 1491176363 & ZNF623 & 8 & 1 & 29 & ins $\mathrm{T}$ & $\# 3$ \\
\hline $1492082552^{*}$ & RECQL4 & 8 & 14 & 20 & delG & $\# 3$ \\
\hline 1498992866 & LOC645969 & 9 & 1 & 155 & insT & $\# 4$ \\
\hline $1527437913^{*}$ & C9orf144B & 9 & 4 & 20 & delC & $\# 4$ \\
\hline $1543290663^{*}$ & FOXD4L5 & 9 & 1 & 39 & delG & $\# 1$ \\
\hline 1546032104 & TRPM3 & 9 & 22 & 19 & insT & \#3 \\
\hline 1552818643 & VPS13A & 9 & 48 & 29 & insG & $\# 3$ \\
\hline 1574648314 & COL15A1 & 9 & 13 & 29 & insC & $\# 3$ \\
\hline 1586295095 & MUSK & 9 & 1 & 62 & ins $\mathrm{T}$ & $\# 3$ \\
\hline $1608846803^{*}$ & $\mathrm{ABO}$ & 9 & 6 & 117 & insC & $\# 4$ \\
\hline 1620006324 & GDI2 & 10 & 7 & 14 & insG & $\# 3$ \\
\hline 1620254092 & IL2RA & 10 & 4 & 14 & insC & $\# 3$ \\
\hline $1621795546^{*}$ & ITIH5 & 10 & 14 & 23 & delC & $\# 1$ \\
\hline 1633127998 & NSUN6 & 10 & 2 & 26 & insA & $\# 3$ \\
\hline 1647389817 & ITGB1 & 10 & 13 & 22 & insA & $\# 3$ \\
\hline $1652560241^{*}$ & LOC340947 & 10 & 2 & 25 & delT & $\# 1$ \\
\hline $1653671683^{*}$ & LOC642424 & 10 & 3 & 117 & delT & $\# 1$ \\
\hline 1657313101 & AGAP4 & 10 & 7 & 23 & delT & $\# 2$ \\
\hline 1658942495 & FAM25G & 10 & 3 & 48 & insC & $\# 3$ \\
\hline 1662197526 & LOC10028793 & 10 & 6 & 22 & insA & $\# 4$ \\
\hline $1662338998^{*}$ & AGAP6 & 10 & 1 & 50 & insC & $\# 2$ \\
\hline 1666373362 & PCDH15 & 10 & 19 & 56 & insC & $\# 3$ \\
\hline 1673760921 & TMEM26 & 10 & 6 & 25 & ins $\mathrm{T}$ & $\# 3$ \\
\hline 1685560504 & FAM149B1 & 10 & 7 & 26 & insT & $\# 3$ \\
\hline 1701949711 & PANK1 & 10 & 3 & 30 & insA & $\# 3$ \\
\hline 1708407108 & CCNJ & 10 & 3 & 18 & insC & $\# 3$ \\
\hline 1708510568 & ZNF518A & 10 & 1 & 30 & insC & $\# 1$ \\
\hline 1708668598 & DNTT & 10 & 2 & 18 & insA & $\# 3$ \\
\hline 1709332414 & C10orf12 & 10 & 1 & 18 & insG & $\# 3$ \\
\hline $1728973932^{*}$ & PNLIPRP2 & 10 & 3 & 52 & insG & $\# 1$ \\
\hline 1733216553 & BRWD2 & 10 & 8 & 28 & insT & $\# 3$ \\
\hline 1737221823 & ZRANB1 & 10 & 1 & 17 & insA & $\# 3$ \\
\hline 1738045786 & MMP21 & 10 & 7 & 33 & insG & $\# 3$ \\
\hline 1748226082 & C11orf21 & 11 & 4 & 63 & insG & $\# 1$ \\
\hline
\end{tabular}




\begin{tabular}{|c|c|c|c|c|c|c|}
\hline 1750053615 & RRM1 & 11 & 14 & 17 & ins $\mathrm{T}$ & $\# 3$ \\
\hline 1753342600 & SYT9 & 11 & 4 & 18 & insG & $\# 3$ \\
\hline $1760006709^{*}$ & SPON1 & 11 & 5 & 72 & insC & $\# 4$ \\
\hline 1764016157 & SAAL1 & 11 & 7 & 40 & delT & $\# 4$ \\
\hline 1771005317 & LUZP2 & 11 & 12 & 34 & insA & $\# 3$ \\
\hline 1782417168 & TRAF6 & 11 & 6 & 24 & delG & \#3 \\
\hline 1782519665 & RAG2 & 11 & 1 & 21 & insA & $\# 3$ \\
\hline $1792247476^{*}$ & CREB3L1 & 11 & 12 & 40 & insG & $\# 1$ \\
\hline 1802120506 & TCN1 & 11 & 7 & 13 & insA & $\# 3$ \\
\hline $1802663567^{*}$ & MS4A14 & 11 & 2 & 61 & delTT & $\# 4$ \\
\hline $1802663568^{*}$ & MS4A14 & 11 & 2 & 22 & delT & $\# 3$ \\
\hline $1803663946^{*}$ & TMEM216 & 11 & 3 & 54 & insA & $\# 4$ \\
\hline 1804797590 & AHNAK & 11 & 3 & 12 & insG & $\# 3$ \\
\hline 1805556025 & SLC22A10 & 11 & 1 & 17 & insC & \#3 \\
\hline $1810263379^{*}$ & UNC93B1 & 11 & 7 & 53 & insG & $\# 3$ \\
\hline $1810284280^{*}$ & ALDH3B1 & 11 & 2 & 63 & insC & $\# 2$ \\
\hline $1810287509^{*}$ & ALDH3B1 & 11 & 6 & 18 & $\mathrm{insC}$ & $\# 1$ \\
\hline $1810293595^{*}$ & ALDH3B1 & 11 & 9 & 28 & $\mathrm{ins} \mathrm{C}$ & $\# 4$ \\
\hline 1814065554 & LOC 729523 & 11 & 1 & 22 & delT & $\# 3$ \\
\hline 1826743977 & DLG2 & 11 & 5 & 23 & ins $\mathrm{T}$ & \#3 \\
\hline 1832107207 & LOC642446 & 11 & 1 & 33 & delT & $\# 4$ \\
\hline $1837197723^{*}$ & CWC15 & 11 & 5 & 152 & inst & $\# 1$ \\
\hline $1837299118^{*}$ & SFRS2B & 11 & 1 & 36 & insC & $\# 4$ \\
\hline 1850549218 & ATM & 11 & 49 & 24 & inst & \#3 \\
\hline 1852355678 & $\mathrm{ZC} 3 \mathrm{H} 12 \mathrm{C}$ & 11 & 2 & 25 & $\mathrm{ins} \mathrm{C}$ & $\# 3$ \\
\hline $1854201323^{*}$ & DIXDC1 & 11 & 7 & 16 & insC & $\# 1$ \\
\hline $1860877259^{*}$ & TREH & 11 & 15 & 28 & insG & $\# 2$ \\
\hline $1861246651^{*}$ & SLC37A4 & 11 & 3 & 37 & delC & $\# 1$ \\
\hline 1861288156 & VPS11 & 11 & 2 & 13 & insC & $\# 4$ \\
\hline 1867800518 & EI24 & 11 & 9 & 14 & $\operatorname{insC}$ & $\# 4$ \\
\hline 1867851321 & CHEK1 & 11 & 5 & 44 & ins C & \#3 \\
\hline $1888645169^{*}$ & PRB3 & 12 & 4 & 34 & delG & $\# 4$ \\
\hline $1888731023^{*}$ & PRB1 & 12 & 3 & 136 & delC & $\# 1$ \\
\hline 1891856090 & ATF7IP & 12 & 11 & 19 & insG & $\# 3$ \\
\hline $1893735417^{*}$ & MGST1 & 12 & 2 & 12 & delAA & \#3 \\
\hline $1893735418^{*}$ & MGST1 & 12 & 2 & 18 & delA & \#3 \\
\hline
\end{tabular}




\begin{tabular}{|c|c|c|c|c|c|c|}
\hline 1898574937 & SLCO1B1 & 12 & 7 & 17 & ins $\mathrm{C}$ & $\# 3$ \\
\hline 1902256413 & BCAT1 & 12 & 5 & 22 & insG & $\# 3$ \\
\hline 1913975525 & KIF21A & 12 & 10 & 20 & ins T & $\# 3$ \\
\hline 1914378775 & SLC2A13 & 12 & 10 & 17 & insA & $\# 3$ \\
\hline 1927092176 & KRT6C & 12 & 1 & 15 & insG & $\# 2$ \\
\hline 1930622534 & SUOX & 12 & 3 & 14 & insG & $\# 3$ \\
\hline 1931678522 & TMEM194A & 12 & 9 & 23 & insG & \#3 \\
\hline 1932337710 & Os9 & 12 & 12 & 17 & insA & \#3 \\
\hline 1959863488 & LRRIQ1 & 12 & 26 & 12 & delA & $\# 3$ \\
\hline 1962616654 & C12orf50 & 12 & 3 & 28 & insA & $\# 3$ \\
\hline $1978598568^{*}$ & TDG & 12 & 3 & 14 & insA & $\# 3$ \\
\hline 1986789153 & LOC100287839 & 12 & 9 & 35 & ins $\mathrm{C}$ & $\# 3$ \\
\hline 1997115077 & RSRC2 & 12 & 10 & 28 & insG & $\# 3$ \\
\hline 1999523126 & $\mathrm{UBC}$ & 12 & 1 & 29 & delT & $\# 3$ \\
\hline 2009256491 & ZMYM5 & 13 & 5 & 14 & ins $\mathrm{C}$ & $\# 3$ \\
\hline 2012756904 & SACS & 13 & 9 & 20 & insT & $\# 3$ \\
\hline 2012761230 & SACS & 13 & 9 & 23 & insT & $\# 3$ \\
\hline 2017859185 & FLT1 & 13 & 4 & 36 & insA & $\# 3$ \\
\hline 2022550582 & STARD13 & 13 & 5 & 85 & delT & $\# 1$ \\
\hline 2026525487 & CSNK1A1L & 13 & 1 & 13 & ins $\mathrm{C}$ & $\# 2$ \\
\hline 2038965626 & RCBTB1 & 13 & 8 & 17 & insG & $\# 3$ \\
\hline 2046563396 & PRR20 & 13 & 2 & 28 & delC & $\# 2$ \\
\hline 2063234131 & KLF12 & 13 & 4 & 47 & insT & $\# 3$ \\
\hline 2066482633 & MYCBP2 & 13 & 75 & 17 & insT & $\# 3$ \\
\hline 2066508358 & MYCBP2 & 13 & 62 & 14 & insC & \#3 \\
\hline 2066632819 & MYCBP2 & 13 & 22 & 23 & delC & $\# 3$ \\
\hline 2066717508 & MYCBP2 & 13 & 2 & 33 & delAA & $\# 3$ \\
\hline 2066717509 & MYCBP2 & 13 & 2 & 33 & delA & $\# 3$ \\
\hline 2088603872 & GPR18 & 13 & 1 & 20 & ins $\mathrm{T}$ & \#3 \\
\hline 2105948032 & NDRG2 & 14 & 1 & 34 & delG & $\# 1$ \\
\hline 2106009532 & FLJ10357 & 14 & 18 & 14 & delG & $\# 3$ \\
\hline 2108927297 & DHRS4L2 & 14 & 6 & 41 & insA & $\# 4$ \\
\hline $2109139875^{*}$ & MDP-1 & 14 & 6 & 13 & delA & $\# 1$ \\
\hline 2117359342 & AKAP6 & 14 & 1 & 20 & insA & $\# 3$ \\
\hline 2117747539 & AKAP6 & 14 & 12 & 21 & insA & $\# 3$ \\
\hline 2137979011 & DDHD1 & 14 & 10 & 22 & insC & $\# 3$ \\
\hline
\end{tabular}




\begin{tabular}{|c|c|c|c|c|c|c|}
\hline $2148241015^{*}$ & GPHB5 & 14 & 1 & 18 & insG & $\# 4$ \\
\hline $2158414589^{*}$ & C14orf169 & 14 & 1 & 19 & insC & \#3 \\
\hline $2159993929^{*}$ & FAM164C & 14 & 1 & 14 & insA & $\# 1$ \\
\hline 2160606560 & TTLL5 & 14 & 4 & 17 & insA & $\# 3$ \\
\hline 2179419547 & SERPINA12 & 14 & 2 & 54 & ins $\mathrm{C}$ & $\# 3$ \\
\hline 2179491154 & SERPINA4 & 14 & 3 & 14 & insG & $\# 3$ \\
\hline 2181450460 & PAPOLA & 14 & 5 & 33 & insC & $\# 3$ \\
\hline $2202211427^{*}$ & CHRFAM7A & 15 & 4 & 191 & delCA & $\# 1$ \\
\hline $2202211428^{*}$ & CHRFAM7A & 15 & 4 & 252 & delA & $\# 4$ \\
\hline $2203996021^{*}$ & CHRNA7 & 15 & 6 & 166 & delTG & $\# 1$ \\
\hline $2203996022^{*}$ & CHRNA7 & 15 & 6 & 50 & delG & $\# 2$ \\
\hline 2204534873 & SCG5 & 15 & 5 & 24 & insC & $\# 3$ \\
\hline 2212460825 & CASC5 & 15 & 10 & 14 & insA & $\# 3$ \\
\hline 2220067652 & SLC12A1 & 15 & 5 & 21 & insA & $\# 3$ \\
\hline 2237036677 & LOC100287371 & 15 & 3 & 32 & insG & $\# 3$ \\
\hline $2243652079^{*}$ & NR2E3 & 15 & 6 & 34 & delC & $\# 1$ \\
\hline 2251295853 & KIAA1024 & 15 & 1 & 14 & inst & $\# 3$ \\
\hline 2252413491 & ARNT2 & 15 & 14 & 24 & insC & $\# 3$ \\
\hline 2256610001 & ZSCAN2 & 15 & 2 & 14 & insC & $\# 3$ \\
\hline 2257065252 & PDE8A & 15 & 4 & 20 & delT & $\# 3$ \\
\hline 2261248094 & FANCI & 15 & 2 & 19 & insC & $\# 3$ \\
\hline 2261584966 & C15orf42 & 15 & 7 & 21 & inst & $\# 3$ \\
\hline $2270957952^{*}$ & LOC145814 & 15 & 4 & 23 & insC & $\# 4$ \\
\hline $2271092254^{*}$ & SYNM & 15 & 1 & 19 & insG & $\# 3$ \\
\hline $2274046312^{*}$ & C16orf35 & 16 & 12 & 89 & insG & $\# 4$ \\
\hline 2274304546 & AXIN1 & 16 & 1 & 20 & delC & $\# 3$ \\
\hline $2277509768^{*}$ & NLRC3 & 16 & 7 & 81 & delG & $\# 1$ \\
\hline $2285935013^{*}$ & LOC729978 & 16 & 4 & 20 & delAT & $\# 4$ \\
\hline $2285935014^{*}$ & LOC729978 & 16 & 4 & 44 & delT & $\# 1$ \\
\hline 2292434768 & NOMO2 & 16 & 24 & 22 & insG & $\# 3$ \\
\hline 2294397443 & ACSM2A & 16 & 9 & 23 & delA & $\# 3$ \\
\hline 2294883814 & DNAH3 & 16 & 53 & 18 & insC & $\# 3$ \\
\hline 2304906998 & HSD3B7 & 16 & 6 & 48 & delC & $\# 2$ \\
\hline 2332868773 & CLEC18C & 16 & 3 & 24 & insA & $\# 3$ \\
\hline $2333553555^{*}$ & HYDIN & 16 & 68 & 29 & delA & $\# 4$ \\
\hline $2338969142^{*}$ & CNTNAP4 & 16 & 1 & 82 & ins $\mathrm{T}$ & $\# 1$ \\
\hline
\end{tabular}




\begin{tabular}{|c|c|c|c|c|c|c|}
\hline $2351412465^{*}$ & LOC100289580 & 16 & 2 & 67 & delC & $\# 2$ \\
\hline 2354387432 & PRPF8 & 17 & 4 & 11 & insG & $\# 3$ \\
\hline $2356396572^{*}$ & P2RX5 & 17 & 3 & 13 & delG & $\# 1$ \\
\hline 2359357840 & C17orf100 & 17 & 1 & 14 & insG & $\# 2$ \\
\hline $2360272579 *$ & SENP3 & 17 & 6 & 20 & delA & \#4 \\
\hline $2361527508^{*}$ & PIK3R6 & 17 & 16 & 42 & insG & $\# 1$ \\
\hline 2363416732 & C17orf48 & 17 & 3 & 19 & insA & $\# 3$ \\
\hline 2371198121 & LGALS9C & 17 & 9 & 16 & insA & $\# 4$ \\
\hline $2376394518^{*}$ & SEBOX & 17 & 1 & 29 & insG & $\# 4$ \\
\hline $2376430014^{*}$ & SLC46A1 & 17 & 4 & 15 & delA & $\# 1$ \\
\hline 2382300534 & CCL7 & 17 & 2 & 24 & insT & $\# 3$ \\
\hline $2383802642^{*}$ & MMP28 & 17 & 4 & 28 & ins $\mathrm{C}$ & $\# 4$ \\
\hline 2384283858 & TBC1D3C & 17 & 13 & 31 & insG & $\# 1$ \\
\hline 2388631071 & KRT10 & 17 & 1 & 14 & delC & $\# 3$ \\
\hline $2392844842^{*}$ & PLCD3 & 17 & 10 & 24 & ins $\mathrm{C}$ & $\# 1$ \\
\hline $2393016586^{*}$ & MAP3K14 & 17 & 4 & 16 & insG & $\# 2$ \\
\hline 2407377091 & CLTC & 17 & 3 & 28 & inst & $\# 3$ \\
\hline 2409792732 & MED13 & 17 & 2 & 24 & insA & $\# 3$ \\
\hline $2411313186^{*}$ & WDR68 & 17 & 5 & 42 & delG & $\# 1$ \\
\hline 2412151377 & DDX5 & 17 & 8 & 23 & insT & $\# 3$ \\
\hline 2434290839 & MYOM1 & 18 & 8 & 16 & insA & $\# 3$ \\
\hline 2448603454 & RBBP\& & 18 & 14 & 22 & insC & $\# 3$ \\
\hline 2451555232 & LOC100287386 & 18 & 2 & 31 & insA & $\# 1$ \\
\hline 2471234235 & SLC14A2 & 18 & 4 & 39 & delC & $\# 3$ \\
\hline 2492044315 & CDH19 & 18 & 11 & 24 & insA & $\# 3$ \\
\hline 2501962862 & ZNF516 & 18 & 2 & 27 & delG & $\# 2$ \\
\hline $2508173565^{*}$ & SPPL2B & 19 & 7 & 26 & ins $\mathrm{C}$ & $\# 2$ \\
\hline 2510788089 & UHRF1 & 19 & 14 & 13 & ins $\mathrm{C}$ & $\# 3$ \\
\hline 2514792389 & MUC16 & 19 & 3 & 18 & insA & $\# 3$ \\
\hline 2514803399 & MUC16 & 19 & 3 & 29 & ins $\mathrm{T}$ & $\# 3$ \\
\hline 2518236406 & ZNF799 & 19 & 4 & 25 & insA & $\# 3$ \\
\hline $2521463907^{*}$ & CYP4F8 & 19 & 4 & 79 & insC & $\# 1$ \\
\hline $2522001621^{*}$ & $\mathrm{HSH} 2 \mathrm{D}$ & 19 & 5 & 71 & delA & $\# 2$ \\
\hline 2538892348 & C19orf55 & 19 & 9 & 17 & delG & $\# 2$ \\
\hline 2543188059 & ZNF780B & 19 & 2 & 24 & insC & \#3 \\
\hline $2543756504^{*}$ & LTBP4 & 19 & 24 & 14 & insG & $\# 1$ \\
\hline
\end{tabular}




\begin{tabular}{|c|c|c|c|c|c|c|}
\hline $2544255517^{*}$ & CYP2F1 & 19 & 1 & 53 & insC & $\# 4$ \\
\hline 2544853028 & CEACAM5 & 19 & 4 & 26 & ins $\mathrm{T}$ & $\# 3$ \\
\hline $2547650400^{*}$ & CEACAM20 & 19 & 8 & 54 & delT & $\# 1$ \\
\hline $2547930257^{*}$ & CBLC & 19 & 8 & 18 & ins $C$ & $\# 3$ \\
\hline $2552076265^{*}$ & DHDH & 19 & 4 & 55 & insG & $\# 2$ \\
\hline 2552600822 & ALDH16A1 & 19 & 10 & 73 & insC & $\# 2$ \\
\hline $2554469302^{*}$ & LOC 147645 & 19 & 10 & 37 & insG & $\# 4$ \\
\hline $2555437083^{*}$ & ZNF480 & 19 & 1 & 51 & delG & $\# 1$ \\
\hline 2555750854 & ZNF83 & 19 & 1 & 26 & insG & $\# 3$ \\
\hline 2559350849 & ZSCAN5C & 19 & 1 & 50 & insA & $\# 2$ \\
\hline 2560590155 & ZNF749 & 19 & 3 & 34 & insA & $\# 1$ \\
\hline 2560866399 & ZNF671 & 19 & 4 & 14 & insA & $\# 3$ \\
\hline $2561351770^{*}$ & ZNF274 & 19 & 4 & 78 & insG & $\# 2$ \\
\hline 2562070563 & DEFB126 & 20 & 2 & 20 & delCC & $\# 3$ \\
\hline 2562070564 & DEFB126 & 20 & 2 & 20 & delC & $\# 3$ \\
\hline $2567847490^{*}$ & CHGB & 20 & 4 & 28 & delGA & $\# 2$ \\
\hline $2567847491^{*}$ & $\mathrm{CHGB}$ & 20 & 4 & 70 & delA & $\# 2$ \\
\hline 2580083985 & CSRP2BP & 20 & 4 & 17 & insG & $\# 3$ \\
\hline $2583130413^{*}$ & NCRNA00153 & 20 & 7 & 49 & insG & $\# 1$ \\
\hline 2606534089 & DDX27 & 20 & 4 & 21 & insA & $\# 3$ \\
\hline 2608270909 & MOCS3 & 20 & 1 & 23 & insG & $\# 3$ \\
\hline $2640900547^{*}$ & KRTAP7-1 & 21 & 1 & 27 & delA & $\# 4$ \\
\hline $2643647262^{*}$ & SON & 21 & 12 & 40 & insA & $\# 1$ \\
\hline $2643647273^{*}$ & SON & 21 & 12 & 33 & delA & $\# 4$ \\
\hline 2654166830 & TRAPPC10 & 21 & 21 & 24 & inst & $\# 3$ \\
\hline $2656193953^{*}$ & OC100288508 & 21 & 5 & 14 & insC & $\# 1$ \\
\hline 2670991039 & HORMAD2 & 22 & 2 & 23 & insG & $\# 3$ \\
\hline 2681753989 & DNAJB7 & 22 & 1 & 66 & insA & $\# 1$ \\
\hline 2683020374 & CYP2D6 & 22 & 5 & 18 & insG & $\# 4$ \\
\hline 2701397266 & WWC3 & $\mathrm{x}$ & 7 & 15 & insG & $\# 3$ \\
\hline 2708217926 & RBBP7 & $\mathrm{x}$ & 2 & 16 & insC & \#3 \\
\hline 2709924320 & CDKL5 & $\mathrm{x}$ & 4 & 26 & $\mathrm{ins} \mathrm{C}$ & $\# 3$ \\
\hline 2711314268 & CXorf23 & $\mathrm{x}$ & 3 & 22 & delG & $\# 3$ \\
\hline 2713575280 & PHEX & $\mathrm{x}$ & 19 & 29 & insG & $\# 3$ \\
\hline 2736210225 & KDM6A & $\mathrm{x}$ & 17 & 17 & $\mathrm{insC}$ & \#3 \\
\hline 2736225869 & KDM6A & $\mathrm{x}$ & 24 & 16 & insA & \#3 \\
\hline
\end{tabular}




\begin{tabular}{|c|c|c|c|c|c|c|}
\hline 2737802282 & SLC9A7 & $\mathrm{X}$ & 7 & 21 & insC & $\# 3$ \\
\hline 2739406485 & SSX1 & $\mathrm{X}$ & 6 & 45 & insT & $\# 3$ \\
\hline $2739444455^{*}$ & SSX9 & $\mathrm{X}$ & 2 & 11 & delC & $\# 2$ \\
\hline $2741301867^{*}$ & DGKK & $\mathrm{X}$ & 22 & 55 & insG & $\# 1$ \\
\hline 2743920170 & SSX2B & $\mathrm{X}$ & 6 & 35 & insC & $\# 3$ \\
\hline 2745406637 & WNK3 & $\mathrm{X}$ & 16 & 40 & insA & $\# 3$ \\
\hline 2755460714 & OPHN1 & $\mathrm{X}$ & 8 & 18 & insC & $\# 3$ \\
\hline 2757668459 & KIF4A & $\mathrm{X}$ & 28 & 24 & insG & $\# 3$ \\
\hline 2758547967 & NONO & $\mathrm{X}$ & 6 & 22 & insT & $\# 3$ \\
\hline 2761842615 & RLIM & $\mathrm{X}$ & 3 & 18 & insG & $\# 3$ \\
\hline 2771580011 & HDX & $\mathrm{x}$ & 5 & 18 & insA & $\# 3$ \\
\hline 2779112744 & PCDH11X & $\mathrm{X}$ & 2 & 18 & insT & $\# 3$ \\
\hline 2788398590 & CENPI & $\mathrm{X}$ & 20 & & insC & $\# 3$ \\
\hline 2789376503 & TCEAL6 & $\mathrm{X}$ & 1 & 24 & insG & $\# 2$ \\
\hline 2789554171 & NXF2 & $\mathrm{X}$ & 7 & 14 & insT & $\# 3$ \\
\hline 2789554906 & NXF2 & $\mathrm{X}$ & 10 & 29 & insA & $\# 3$ \\
\hline 2802179110 & IL13RA2 & $\mathrm{X}$ & 4 & 18 & ins $\mathrm{T}$ & $\# 3$ \\
\hline 2823639589 & ARHGEF6 & $\mathrm{X}$ & 18 & 16 & insA & $\# 3$ \\
\hline $2840930428^{*}$ & LCAP & $\mathrm{X}$ & 1 & 57 & insC & $\# 2$ \\
\hline 2841794077 & MPP1 & $\mathrm{X}$ & 7 & 14 & insG & $\# 3$ \\
\hline
\end{tabular}

*: These indels commonly occurred in more than one HCC.

$\mathrm{a}$ : chromosome, b: coding sequence 
Supplemental Table 6. List of 81 nucleotide positions in 77 genes with indels at a frequency of more than $20 \%$ of reads in 4 non-tumorous tissues of 4 cases.

\begin{tabular}{|c|c|c|c|c|c|c|}
\hline Reference Position & Gene & $\mathrm{Chr}^{\mathrm{a}}$ & $\mathrm{CDS}^{\mathrm{b}}$ & Coverage & Allele change & Case \\
\hline 36247083 & THRAP3 & 1 & 4 & 18 & delG & $\# 1$ \\
\hline 75174421 & SLC44A5 & 1 & 16 & 19 & delT & $\# 1$ \\
\hline 114430296 & TRIM33 & 1 & 20 & 23 & delC & $\# 1$ \\
\hline 133132499 & YY1AP1 & 1 & 7 & 37 & inst & $\# 1$ \\
\hline 133844355 & RHBG & 1 & 9 & 48 & delC & $\# 4$ \\
\hline 201121914 & CAPN2 & 1 & 3 & 14 & delC & $\# 1$ \\
\hline 201173637 & TP53BP2 & 1 & 13 & 22 & delG & $\# 1$ \\
\hline 247664301 & C2orf43 & 2 & 4 & 18 & delA & $\# 2$ \\
\hline 319394229 & SNRNP200 & 2 & 37 & 2 & delA & $\# 1$ \\
\hline 322653290 & AFF3 & 2 & 14 & 35 & delA & $\# 1$ \\
\hline 331834049 & RANBP2 & 2 & 20 & 16 & delG & $\# 1$ \\
\hline 332901065 & RGPD5 & 2 & 20 & 22 & delT & $\# 1$ \\
\hline 374789589 & NEB & 2 & 4 & 37 & ins $\mathrm{T}$ & $\# 4$ \\
\hline 382950536 & LY75 & 2 & 5 & 19 & delA & $\# 4$ \\
\hline 401635744 & TTN & 2 & 274 & 25 & delA & $\# 1$ \\
\hline 409835043 & FAM171B & 2 & 8 & 17 & delT & $\# 1$ \\
\hline 412064501 & COL3A1 & 2 & 14 & 21 & insA & $\# 4$ \\
\hline 454784716 & PTMA & 2 & 4 & 14 & delT & $\# 1$ \\
\hline $463724350^{*}$ & AQP12B & 2 & 1 & 27 & delC & $\# 3$ \\
\hline 463734336 & AQP12A & 2 & 2 & 14 & delG & $\# 2$ \\
\hline 503335742 & DLEC1 & 3 & 4 & 20 & delT & $\# 1$ \\
\hline 503335743 & DLEC1 & 3 & 4 & 20 & delA & $\# 1$ \\
\hline 735406533 & CNOT6L & 4 & 10 & 21 & delG & $\# 1$ \\
\hline 785877214 & LARP2 & 4 & 14 & 23 & delA & $\# 1$ \\
\hline 798021293 & $\mathrm{scOC}$ & 4 & 1 & 18 & insC & $\# 1$ \\
\hline 810971969 & TRIM2 & 4 & 5 & 18 & delC & $\# 1$ \\
\hline 883256725 & PRLR & 5 & 3 & 29 & delG & $\# 4$ \\
\hline 939146286 & ANKRD32 & 5 & 16 & 15 & insC & $\# 1$ \\
\hline $985314450^{*}$ & LOC100288105 & 5 & 1 & 27 & delC & $\# 3$ \\
\hline 1033746568 & BMP6 & 6 & 5 & 24 & delC & $\# 1$ \\
\hline 1068664364 & KIAA0240 & 6 & 4 & 17 & ins $\mathrm{T}$ & $\# 4$ \\
\hline 1193244025 & FAM120B & 6 & 1 & 43 & insA & $\# 1$ \\
\hline
\end{tabular}




\begin{tabular}{|c|c|c|c|c|c|c|}
\hline 1222659823 & KIAA0644 & 7 & 1 & 463 & delC & $\# 4$ \\
\hline 1282877394 & CDK6 & 7 & 3 & 21 & delA & $\# 1$ \\
\hline 1289880816 & CYP3A4 & 7 & 12 & 45 & delG & $\# 1$ \\
\hline 1333766765 & LOC441294 & 7 & 1 & 13 & delA & $\# 4$ \\
\hline 1340012514 & SSPO & 7 & 76 & 53 & delA & \#3 \\
\hline 1356372261 & XKR5 & 8 & 6 & 130 & delA & $\# 4$ \\
\hline $1490189877^{*}$ & JRK & 8 & 1 & 29 & delC & \#3 \\
\hline $1490189878^{*}$ & JRK & 8 & 1 & 15 & delA & \#2 \\
\hline $1492082552^{*}$ & RECQL4 & 8 & 14 & 43 & delG & \#3 \\
\hline 1505961686 & MPDZ & 9 & 2 & 28 & insG & $\# 4$ \\
\hline 1526015264 & NFX1 & 9 & 3 & 36 & delT & $\# 1$ \\
\hline 1573925487 & GABBR2 & 9 & 17 & 59 & insT & $\# 4$ \\
\hline 1580509237 & ABCA1 & 9 & 4 & 24 & ins $\mathrm{T}$ & $\# 1$ \\
\hline 1637516682 & ARMC3 & 10 & 18 & 19 & delT & $\# 1$ \\
\hline 1637516683 & ARMC3 & 10 & 18 & 19 & delT & $\# 1$ \\
\hline $1657313100^{*}$ & AGAP4 & 10 & 7 & 19 & delT & $\# 2$ \\
\hline 1657313101* & AGAP4 & 10 & 7 & 14 & delT & $\# 3$ \\
\hline 1807397040 & SYVN1 & 11 & 7 & 15 & insA & $\# 1$ \\
\hline 1832107207 & LOC642446 & 11 & 1 & 18 & delT & $\# 4$ \\
\hline 1855967268 & ZW10 & 11 & 8 & 40 & delC & $\# 1$ \\
\hline 1861246651 & SLC37A4 & 11 & 3 & 143 & delC & \#4 \\
\hline 1884320486 & ATN1 & 12 & 4 & 15 & delA & $\# 1$ \\
\hline 1929584670 & KIAA0748 & 12 & 6 & 31 & delC & $\# 4$ \\
\hline 1955959709 & PPFIA2 & 12 & 18 & 52 & delA & $\# 4$ \\
\hline 1994379801 & CIT & 12 & 17 & 28 & delG & $\# 1$ \\
\hline 1995110709 & DYNLL1 & 12 & 2 & 14 & delG & $\# 1$ \\
\hline 1997144069 & KNTC1 & 12 & 2 & 31 & delC & $\# 4$ \\
\hline 2105624179 & RNASE4 & 14 & 1 & 15 & delC & $\# 1$ \\
\hline 2162358340 & C14orf133 & 14 & 13 & 15 & delT & $\# 1$ \\
\hline 2243652079 & NR2E3 & 15 & 6 & 129 & delC & $\# 4$ \\
\hline 2256057594 & ADAMTSL3 & 15 & 12 & 26 & delT & $\# 1$ \\
\hline $2277509768^{*}$ & NLRC3 & 16 & 7 & 12 & delG & $\# 2$ \\
\hline 2302633200 & EIF3C & 16 & 4 & 18 & delG & $\# 4$ \\
\hline 2303380808 & SULT1A4 & 16 & 3 & 24 & delA & $\# 1$ \\
\hline 2351412465 & LOC100289580 & 16 & 2 & 103 & delC & $\# 4$ \\
\hline 2356396572 & P2RX5 & 17 & 3 & 40 & delG & \#4 \\
\hline
\end{tabular}




\begin{tabular}{lllllll}
2376621991 & SPAG5 & 17 & 3 & 13 & delC & $\# 1$ \\
2386619109 & CCDC49 & 17 & 5 & 14 & delT & $\# 1$ \\
2413869089 & APOH & 17 & 5 & 24 & delC & $\# 1$ \\
2501962862 & ZNF516 & 18 & 2 & 29 & delG & $\# 3$ \\
2507200605 & MUM1 & 19 & 8 & 36 & delG & $\# 1$ \\
2538892348 & C190rf55 & 19 & 9 & 20 & delG & $\# 2$ \\
2565046537 & UBOX5 & 20 & 2 & 15 & delG & $\# 1$ \\
2587599923 & ZNF337 & 20 & 4 & 19 & delT & $\# 1$ \\
2598525448 & ZHX3 & 20 & 1 & 19 & delT & \#1 \\
2625038622 & NRIP1 & 21 & 1 & 24 & delG & $\# 1$ \\
2661518554 & FAM108A5 & 22 & 2 & 13 & delG & \#3 \\
2748277559 & SPIN2B & X & 1 & 13 & delG & $\# 2$ \\
2792445004 & TEX13A & X & 2 & 18 & delC & $\# 3$ \\
\hline
\end{tabular}

*: These indels commonly occurred in more than one HCC.

a: chromosome, b: coding sequence 
Supplemental Table 7. List of 40 somatic mutations with amino acid changes commonly detected in both the tumor (at a frequency of $20 \%<$ of reads) and matched non-tumorous cirrhotic liver (at a frequency of $5 \%<$ of reads) of the same patients. The two genes listed at the top (indicated by bold type) were recurrently mutated in the non-tumorous inflamed livers of two cases.

\begin{tabular}{|c|c|c|c|c|c|c|c|c|}
\hline \multirow[b]{2}{*}{ Gene } & \multirow[b]{2}{*}{$\begin{array}{c}\text { Reference } \\
\text { Position }\end{array}$} & \multirow[b]{2}{*}{$\mathrm{Chr}^{\mathrm{a}}$} & \multirow[b]{2}{*}{$\begin{array}{l}\text { Reference } \\
\text { Nucleotide }\end{array}$} & \multirow[b]{2}{*}{$\begin{array}{l}\text { Mutation } \\
\text { Nucleotide }\end{array}$} & \multicolumn{2}{|c|}{ Tumor } & \multicolumn{2}{|c|}{ Non-tumor } \\
\hline & & & & & $\begin{array}{c}\text { Mutation } \\
\text { frequency } \\
\text { (\%) }\end{array}$ & Case & $\begin{array}{c}\text { Mutation } \\
\text { frequency } \\
(\%)\end{array}$ & Case \\
\hline LEPR & 65548341 & 1 & C & A & 25.8 & $\# 3$ & 15.0 & $\# 3$ \\
\hline & & & & & & & 21.9 & $\# 1$ \\
\hline ZNF408 & 1792629936 & 11 & $T$ & A & 20.4 & $\# 2$ & 16. 0 & $\# 2$ \\
\hline & & & & & & & 15. 8 & $\# 4$ \\
\hline HRNR & 129676984 & 1 & G & $\mathrm{C}$ & 28.9 & $\# 3$ & 5.4 & $\# 3$ \\
\hline PXDN & 228577682 & 2 & G & C & 45.1 & $\# 4$ & 47.2 & $\# 4$ \\
\hline POTEF & 353150970 & 2 & $\mathrm{~T}$ & A & 41.8 & $\# 4$ & 31.0 & $\# 4$ \\
\hline ALPP & 455451136 & 2 & $\mathrm{C}$ & $\mathrm{T}$ & 32.5 & $\# 4$ & 37.5 & $\# 4$ \\
\hline GPR125 & 682521774 & 4 & C & A & 38.1 & $\# 2$ & 40.0 & $\# 2$ \\
\hline HERC6 & 746068457 & 4 & $\mathrm{~T}$ & A & 36.5 & $\# 4$ & 44.9 & $\# 4$ \\
\hline EGFLAM & 886579974 & 5 & $\mathrm{~T}$ & G & 23.3 & $\# 3$ & 5.3 & $\# 3$ \\
\hline $\mathrm{C} 4 \mathrm{~A}$ & 1057829599 & 6 & $\mathrm{~T}$ & G & 25.0 & $\# 2$ & 11.5 & $\# 2$ \\
\hline WISP3 & 1134999625 & 6 & $\mathrm{~T}$ & G & 43.3 & $\# 4$ & 64.3 & $\# 4$ \\
\hline C7orf10 & 1234451360 & 7 & $\mathrm{~T}$ & A & 25.0 & $\# 3$ & 8.3 & $\# 3$ \\
\hline PVRIG & 1290339880 & 7 & $\mathrm{C}$ & $\mathrm{T}$ & 23.5 & $\# 1$ & 21.3 & $\# 1$ \\
\hline MUC17 & 1291200140 & 7 & G & A & 21.2 & $\# 4$ & 12.5 & $\# 4$ \\
\hline PLOD3 & 1291376235 & 7 & G & $\mathrm{C}$ & 48.2 & $\# 4$ & 51.7 & $\# 4$ \\
\hline COL27A1 & 1589933932 & 9 & A & G & 56.8 & $\# 4$ & 54.6 & $\# 4$ \\
\hline AGAP9 & 1658906463 & 10 & $\mathrm{~T}$ & G & 36.7 & $\# 4$ & 16.2 & $\# 4$ \\
\hline POLL & 1713935693 & 10 & G & $\mathrm{T}$ & 44.8 & $\# 4$ & 38.6 & $\# 4$ \\
\hline MUC5AC & 1747183167 & 11 & G & A & 43.9 & $\# 4$ & 43.8 & $\# 4$ \\
\hline MRGPRX3 & 1764064669 & 11 & $\mathrm{~T}$ & $\mathrm{C}$ & 40.0 & $\# 4$ & 42.5 & $\# 4$ \\
\hline TMEM133 & 1843211533 & 11 & A & $\mathrm{C}$ & 59.5 & $\# 4$ & 83.3 & $\# 4$ \\
\hline TMEM123 & 1844621025 & 11 & G & A & 27.3 & $\# 2$ & 7.3 & $\# 2$ \\
\hline TMPRSS4 & 1860336319 & 11 & $\mathrm{C}$ & $\mathrm{T}$ & 54.4 & $\# 4$ & 41.3 & $\# 4$ \\
\hline DHRS4L2 & 2108914889 & 14 & G & $\mathrm{T}$ & 20.5 & $\# 3$ & 11.5 & $\# 3$ \\
\hline
\end{tabular}




\begin{tabular}{|c|c|c|c|c|c|c|c|c|}
\hline GOLGA6C & 2247104814 & 15 & $\mathrm{~A}$ & $\mathrm{~T}$ & 21.7 & $\# 4$ & 9.6 & $\# 4$ \\
\hline PRSS22 & 2276813235 & 16 & $\mathrm{C}$ & $\mathrm{T}$ & 50.0 & \#4 & 36.7 & $\# 4$ \\
\hline FAM38A & 2351390771 & 16 & $\mathrm{C}$ & $\mathrm{T}$ & 21.4 & $\# 4$ & 54.3 & $\# 4$ \\
\hline GGT6 & 2357265990 & 17 & $\mathrm{G}$ & $\mathrm{A}$ & 92.3 & $\# 4$ & 41.7 & $\# 4$ \\
\hline COX10 & 2366897810 & 17 & $\mathrm{C}$ & $\mathrm{T}$ & 55.2 & \#4 & 36.3 & $\# 4$ \\
\hline KIAA0100 & 2376657621 & 17 & $\mathrm{~A}$ & $\mathrm{C}$ & 47.8 & \#4 & 60.0 & $\# 4$ \\
\hline TBC1D3B & 2384202011 & 17 & $\mathrm{C}$ & $\mathrm{T}$ & 63.0 & \#4 & 27.4 & $\# 4$ \\
\hline TBC1D3D & 2385938140 & 17 & $\mathrm{~A}$ & $\mathrm{G}$ & 45.9 & $\# 4$ & 21.0 & $\# 4$ \\
\hline ERBB2 & 2387531879 & 17 & $\mathrm{~A}$ & $\mathrm{G}$ & 66.7 & \#4 & 54.6 & $\# 4$ \\
\hline $\mathrm{CSH} 2$ & 2411602334 & 17 & $\mathrm{C}$ & $\mathrm{T}$ & 90.9 & $\# 4$ & 79.5 & $\# 4$ \\
\hline QRICH2 & 2423941144 & 17 & $\mathrm{~T}$ & $\mathrm{G}$ & 50.0 & \#4 & 60.4 & $\# 4$ \\
\hline MOCOS & 2461870479 & 18 & $\mathrm{~T}$ & $\mathrm{C}$ & 72.0 & $\# 4$ & 62.2 & $\# 4$ \\
\hline CPAMD8 & 2522819358 & 19 & $\mathrm{G}$ & $\mathrm{A}$ & 21.8 & \#3 & 15.2 & $\# 3$ \\
\hline MAP4K1 & 2541732174 & 19 & $\mathrm{G}$ & $\mathrm{A}$ & 36.0 & $\# 4$ & 54.3 & $\# 4$ \\
\hline PSG8 & 2545901763 & 19 & $\mathrm{C}$ & $\mathrm{A}$ & 28.3 & \#3 & 9.5 & $\# 3$ \\
\hline KRTAP12-2 & 2654734983 & 21 & $\mathrm{C}$ & $\mathrm{T}$ & 59.3 & \#4 & 43.5 & $\# 4$ \\
\hline
\end{tabular}

a: chromosome 
Supplemental Table 8. Overview of selected exome sequencing data of 22 patients with HCV infection. Selected exome sequencing of TP53, CTNNB1, and LEPR was performed for 22 non-tumorous cirrhotic liver tissues, $10 \mathrm{HCC}$ tissues, and matched peripheral lymphocytes from each patient. Aligned reads, aligned sequences (bp), and median read depth are shown for each sample.

\begin{tabular}{ccccc}
\hline & & Aligned reads & Aligned sequence (bp) & Median read depth \\
\hline \multirow{2}{*}{ TP53 } & Tumor & 29,334 & $2,035,570$ & $1,476.2$ \\
& Non-tumor & 31,848 & $2,200,641$ & $1,575.3$ \\
& Lymphocytes & 36,690 & $2,539,944$ & $1,917.2$ \\
\hline \multirow{2}{*}{$C T N N B 1$} & Tumor & 90,022 & $6,215,000$ & $2,344.3$ \\
& Non-tumor & 75,785 & $5,282,450$ & $1,991.2$ \\
& Lymphocytes & 100,430 & $7,013,325$ & $2,710.8$ \\
\hline \multirow{2}{*}{ LEPR } & Tumor & 34,328 & $2,390,335$ & 538.3 \\
& Non-tumor & 60,128 & $4,219,089$ & $1,025.6$ \\
& Lymphocytes & 86,830 & $6,085,511$ & $1,423.0$ \\
\hline
\end{tabular}


Supplemental Table 9. Clinical features and overview of deep sequencing data of patients who underwent deep sequencing of the $L E P R$ gene. We determined the sequences of the LEPR gene in the liver of 15 non-cirrhotic HCV-associated chronic hepatitis patients. In addition, normal liver tissues were obtained from 9 liver donors at the time of the operation. Age, gender, aligned reads, aligned sequences (bp), median read depth, and numbers of mutations are shown.

\begin{tabular}{lcc}
\hline & Chronic hepatitis $(\mathrm{n}=15)$ & Normal liver $(\mathrm{n}=\mathbf{9})$ \\
\hline Age & 59.3 & 55.9 \\
Gender (M/F) & $6 / 9$ & $7 / 2$ \\
Aligned reads & 4,290 & 3,956 \\
Aligned sequence (bp) & $1,044,737$ & $1,275,068$ \\
Median read depth & 2,838 & 3,440 \\
Number of Mutations in $\mathbf{L E P R}$ gene & 0 & 0 \\
\hline
\end{tabular}


Supplemental Table 10. Mean body weights and serum levels of insulin, triglyceride, total cholesterol, and ALT of C57BL/KsJ- $d b / d b(d b / d b)$ mice and misty (control) mice after 4 weeks of thioacetamide administration.

\begin{tabular}{lcc}
\hline & $d b / d b$ & control \\
\hline Body Weight $(\mathrm{g})$ & $46.5 \pm 0.6$ & $23.5 \pm 0.4$ \\
Insulin (ng/mL) & $30.6 \pm 28.3$ & $1.6 \pm 0.2$ \\
Triglyceride (mg/dL) & $95.0 \pm 5.0$ & $50.0 \pm 20.0$ \\
Total cholesterol (mg/dL) & $215.0 \pm 15.0$ & $95.0 \pm 15.0$ \\
ALT (IU/L) & $1,325.0 \pm 1,085.0$ & $75.0 \pm 35.0$ \\
\hline
\end{tabular}

All data are presented as mean \pm standard deviation.

ALT: alanine aminotransferase 
Supplemental Table 11. Categorization of the mutated genes detected by whole exome sequencing of the AID-expressing hepatocyte cell line using the KEGG database.

\begin{tabular}{llllll}
\hline Pathway & & & & \\
\hline Metabolic pathways & ATP6V0A4 & DMGDH & HSD17B3 & PGD \\
& ATP6V1C2 & GALNT1 & HYAL2 & PHGDH \\
& BCMO1 & GATM & NDST1 & POLR3B \\
& CPS1 & HKDC1 & PAH & \\
PI3K-Akt signaling pathway & BCL2L11 & IBSP NOS3 & PRKCZ & TEK \\
MAPK signaling pathway & COL27A1 & & & \\
Cytokine-cytokine receptor interaction & FLNB & SP1 & CACNA1F & PTPN7 \\
Transcriptional misregulation in cancer & EYA1 & TNFRSF8 & TNFRSF10A & \\
Proteoglycans in cancer & FLN & ITGB3 & TIMP3 & VTN \\
PPAR signaling pathway & CPT1B & CYP4A22 & PPARD & \\
Cell cycle & E2F2 & ESPL1 & MCM7 & \\
Pathways in cancer & FLT3 & TRAF4 & PDGFA & \\
Hedgehog signaling pathway & GLI3 & LRP2 & CSNK1A1L & \\
Others & 95 genes & & & \\
\hline
\end{tabular}

The genes categorized in multiple pathways are shown in only one representative pathway.

Constitutive AID expression resulted in the accumulation of nucleotide alterations in various genes including LEPR of the cultured hepatocyte-derived cells.

Whole exome sequencing was performed on DNA derived from established non-neoplastic human primary hepatocyte cells (J Hepatol.2007 ;46:26-36.) with constitutive AID expression. AID expression in the cultured hepatocytes was performed using a lentiviral system ${ }^{26}$. After 8 weeks of AID expression, the DNA was extracted and subjected to whole exome sequencing as described in the Materials and Methods. Overall, a total of 460 nucleotide positions in 380 different genes were defined as mutated in the AID-expressing cultured hepatocytes through the variant filtering process. Among them, pathway analyses by KEGG revealed that many genes, including LEPR, were categorized into well-known signaling pathways: metabolic pathway, PI3K-Akt signaling pathway, MAPK signaling pathway, cytokine-cytokine receptor interaction pathway, and the transcriptional misregulation in cancer pathway. Only categorized genes are shown. 


\section{Supplemental Information}

\section{Materials and Methods \\ Patients}

The study group comprised of patients who had undergone living donor liver transplantation or potentially curative resection of primary $\mathrm{HCC}$ at Kyoto University Hospital from 2000 to 2010. The selection of patients enrolled in this study was based on the availability of a sufficient amount of tissue for analysis. Patients included 17 men and 9 women, with a mean age at the time of surgery of $54.9 \pm 7.7$ years (mean \pm SD; range, 37-76 years). Among them, whole exome sequencing was applied to 7 tumors, 4 non-tumorous cirrhotic livers, and matched peripheral lymphocytes from 4 patients (Supplemental Table 1, \#1-4). Furthermore, we performed selected exome sequencing of 22 non-tumorous cirrhotic livers, 10 tumors, and matched peripheral lymphocytes from 22 other affected individuals (Supplemental Table 1, \#5-26). All patients were positive for serum anti-HCV and/or HCV RNA. Written informed consent for the use of resected tissue was obtained from all patients in accordance with the Declaration of Helsinki, and the Kyoto University graduate School and Faculty of Medicine Ethics Committee approved the study.

\section{Sequence data analysis and variant filtering.}

Using the software "NextGENe v2.2"(SoftGenetics, State College, PA), the obtained reads were aligned with the reference sequences of the Human Genome Build 37.3. Reads with $96 \%$ or more bases matching a particular position of the reference sequences were aligned. Furthermore, reads with a median quality value score of more than 20 and no more than 3 uncalled nucleotides were allowed anywhere in one read. Only sequences that passed the quality filters were analyzed and each position of the genome was assigned a coverage depth, representing the number of times the nucleotide position was sequenced. To identify somatic mutations, we used a number of scores to provide an empirical estimation of the likelihood that a given mutation was real and not an artifact of sequencing or alignment errors.

In the whole exome sequencing analysis, candidates of somatic mutations were selected according to the variant filtering process (Supplemental Figure 1). We defined nucleotide alterations that appeared in more than $20 \%$ of reads as somatic mutations ${ }^{18,23 \text {, }}$ 31 . When detecting the genes commonly mutated in both tumor and non-tumorous liver tissues of the same individual, we also selected potential nucleotide alterations that appeared between $5 \%$ and $20 \%$ of the total reads in non-tumorous liver tissues for further evaluation. We excluded potential somatic mutations that represented more than 
$5 \%$ of the reads in peripheral lymphocytes of the same patient as common variants in each individual. Candidate nucleotide alterations were tested using standard Sanger sequencing on an Applied Biosystems 3500 Genetic Analyzer (Applied Biosystems, Foster City, CA) to validate the presence of each mutation.

In selected exome sequencing analysis, candidates of somatic mutations were selected according to the variant filtering process (Supplemental Figure 2). We defined somatic mutations with more than $20 \%$ of reads as high-frequency mutations and those that appeared between $1 \%$ and $20 \%$ of total reads as low-frequency mutations. We excluded potential somatic mutations that represented more than $1 \%$ of the reads in peripheral lymphocytes of the same patient. In cases in which we could not obtain lymphocyte DNA, candidates of somatic mutations found in the lymphocytes of two or more different individuals were excluded in consideration of possible Japanese polymorphisms.

We compared our variants against common and germline polymorphisms present in the dbSNP135 to discard known germline SNPs.

All sequence reads were deposited in the DNA Data Bank of Japan Sequence Read Archive; accession number DRA000867.

\section{Score}

SoftGenetics developed the Overall Mutation score to provide an empirical estimation of the likelihood that a given mutation is real and not an artifact of sequencing or alignment errors. Overall Mutation Score of NextGENe can be used like Phred scores, in which the scores are logarithmically linked to error probabilities. The Overall Mutation Score of NextGENe is obtained as the product of the "Coverage Score", which is calculated from the depth of coverage at the position of the mutation and whose value ranges from zero (0) (where depth of coverage is one (1)) to an unlimited number, multiplied by each of the four types of additional penalty scores such, as the "Read Balance Score", "Allele Balance Score", "Mismatch Score" and "Wrong Allele Score", whose values are less than 1 but are positive (the calculating formula for each score is not shown). These scores are described in the NextGENe User Manual in detail (http://www.softgenetics.com/NextGENe.html).

\section{Overall Mutation score}

SoftGenetics developed the Overall Mutation score to provide an empirical estimation of the likelihood that a given mutation is real and not an artifact of sequencing or alignment errors. A low Overall Mutation score, however, does not mean that the mutation is more than likely a false mutation. The low score implies only that the mutation cannot be called a true mutation with absolute certainty. As a general 
guideline, if the coverage is high (500 to several thousand reads) and the data is bi-directional, then scores that are 5 and lower indicate that the mutation is most likely false, while scores of 25 and higher indicate that the mutation is most likely true.

\section{Mismatch score}

Several variations from the reference sequence that occur very close together often indicate a region where mutation calls are less reliable. The Mismatch score penalizes a specific mutation if other mismatched bases are found nearby. The software first looks for mismatches that occur in a minimum percentage of reads in the $10 \mathrm{bp}$ region that is found on either of side of the mutation that is being scored.

\section{Wrong Allele score}

Mismatches that are different from the consensus are referred to as wrong mismatches. These wrong mismatches most likely result from sequencing errors. For example, A, C, $\mathrm{G}, \mathrm{T}$, and insertions represent wrong mismatches when a deletion was called at a position.

\section{Cell culture and transfection}

The cDNA encoding the wild-type and the mutated LEPR were generated by RT-PCR from the mRNA of the liver tissues, followed by PCR amplification using Phusion High-fidelity DNA polymerase (Finnzymes, Espoo, Finland) and the following oligonucleotide primers: 5'-CGCGGATCCATGATTTGTCAAAAATTC-3' (sense) and 5'- AAGGAAAAAAGCGGCCGCTTACACAGTTAGGTCACACA-3' (antisense). The resulting PCR fragments were inserted into the BamHI-NotI sites of pcDNA3 for HEK293 and the BamHI-ApaI sites of lentivirus for HepG2, as described previously14.

HEK293 and HepG2 cells were maintained in Dulbecco's modified Eagle medium (Gibco-BRL, Rockville, MD) containing 10\% fetal bovine serum. For transfection of plasmids into the HEK293 cells, we used Lipofectamine2000 transfection reagent (Invitrogen, Carlsbad, CA). At 40h post-transfection, the cells were serum starved for $8 \mathrm{~h}$, then either left unstimulated or stimulated with $100 \mathrm{ng} / \mathrm{mL}$ recombinant human leptin (Sigma-Aldrich) for $10 \mathrm{~min}$. Expression of either wild-type or mutant LEPR in HepG2 cells was performed using a lentiviral vector-mediated wild-type and mutated LEPR expression system as described previously26. In brief, LEPR complementary DNA fragments were inserted into the viral vectors, followed by the production of lentiviral stocks in HEK293 cells. HepG2 cells were cultured in virus-containing medium for 48h, starved for $8 \mathrm{~h}$, treated with $100 \mathrm{ng} / \mathrm{mL}$ recombinant human leptin (Sigma-Aldrich) for $10 \mathrm{~min}$, and then subjected to immunoblotting, immunostaining, quantitative RT-PCR, or a cell proliferation (MTT) assay. 


\section{Immunoblotting analysis}

Immunoblotting was performed using anti-signal transducer and activator of transcription 3 (STAT3) and anti-Phospho-STAT3 antibody (Cell Signaling Technology, Danvers, MA) according to the manufacturer's protocol.

\section{Animals Experiments}

C57BL/KsJ- $d b / d b$ mice ( $d b / d b$ mice), which possess homozygous deletion of the Lepr, $O b-R$ gene, and misty mice, which are wild-type with a normal Lepr, were purchased from Japan SLC (Shizuoka, Japan). Thioacetamide (TAA) (Sigma-Aldrich, St. Louis, MO) was prepared at a concentration of $0.02 \%$ and administered to mice in the drinking water for 24 weeks or 30 weeks beginning at 5 weeks of age. These mice were then sacrificed for analysis of the development of liver tumors. All animal experiments were approved by the ethics committee for animal experiments and performed under the Guidelines for Animal Experiments of Kyoto University. 


\section{Patient \#1}

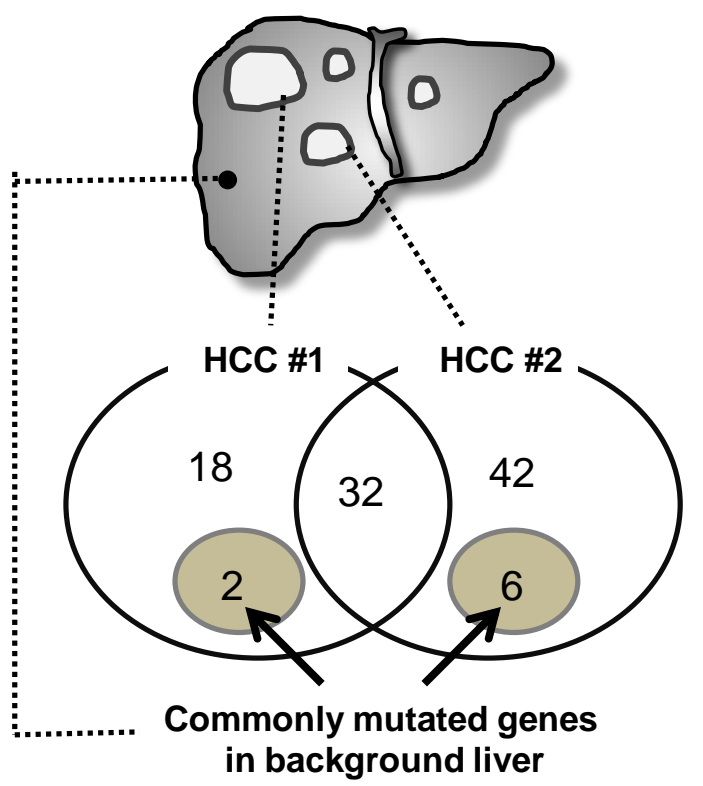

Patient \#3

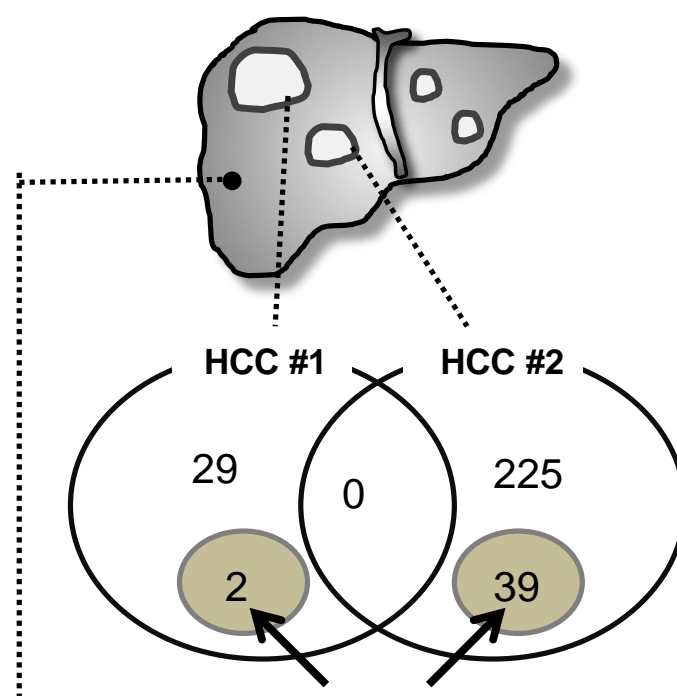

Commonly mutated genes in background liver
Patient \#2

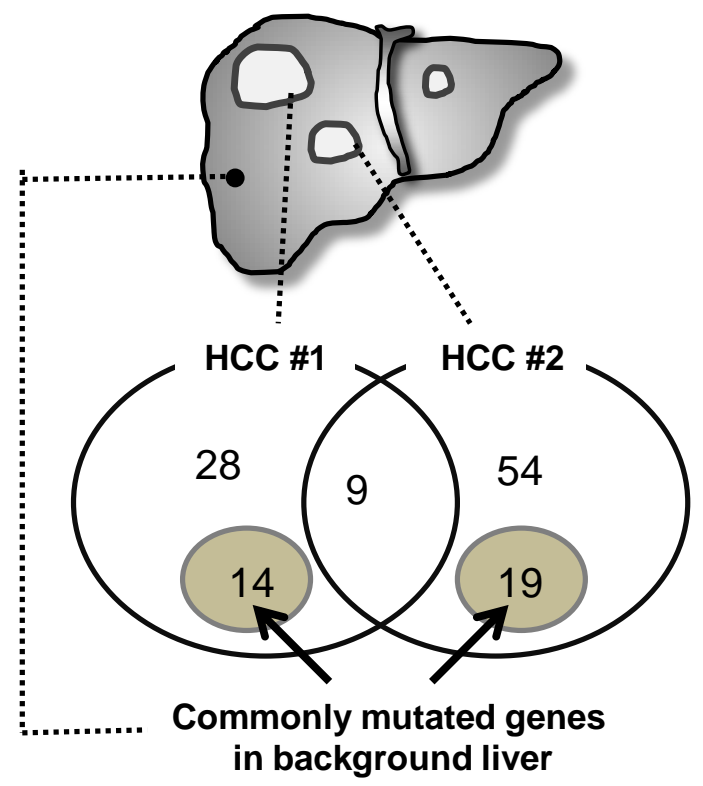

Patient \#4

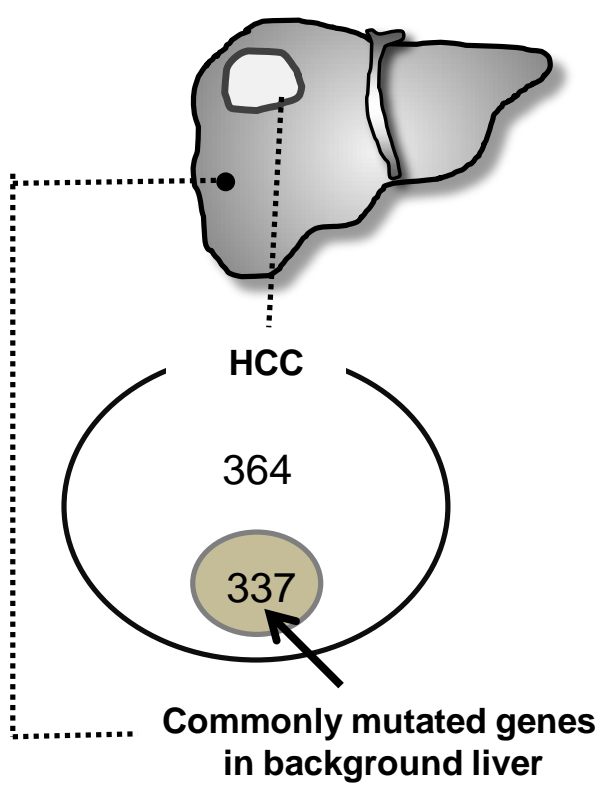




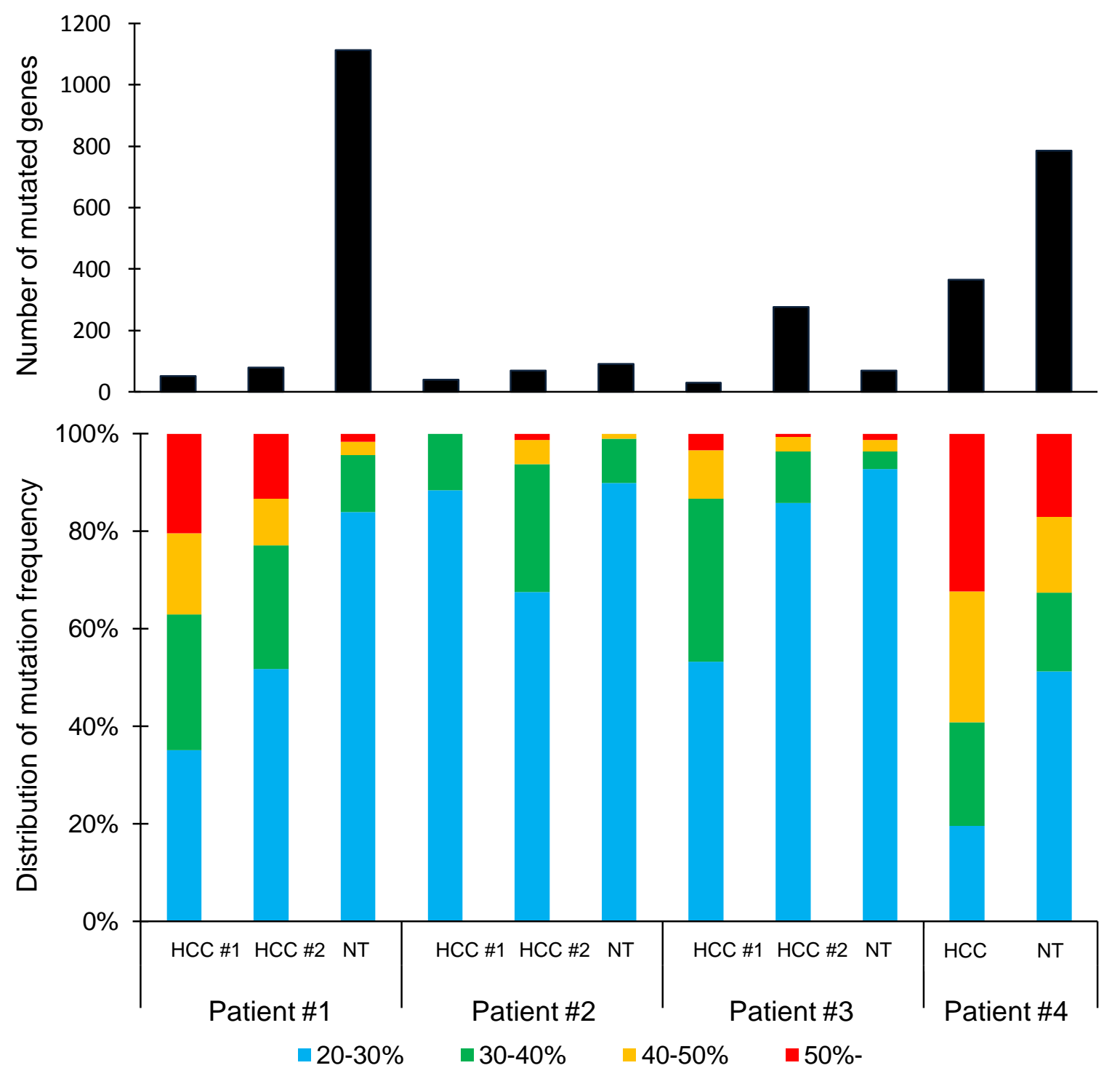


A

\section{LEPR gene}

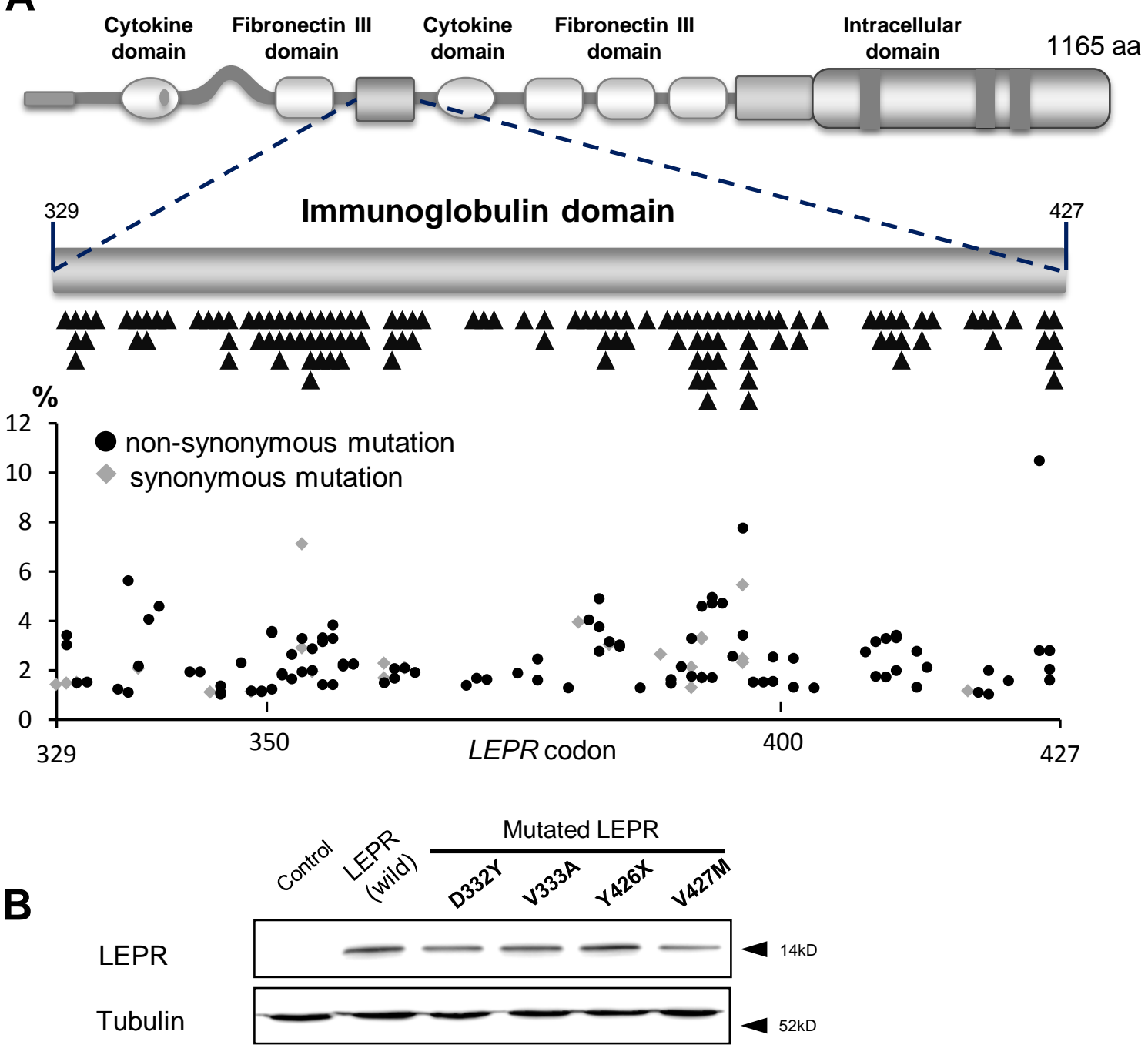

C

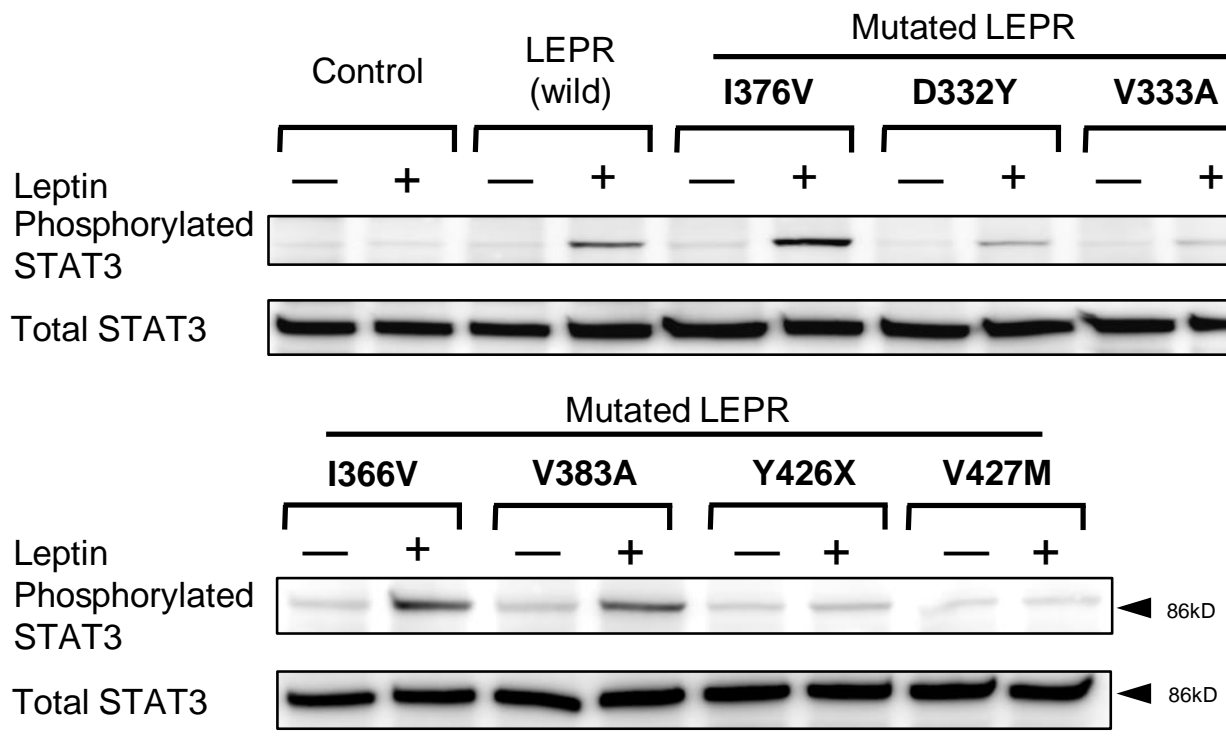


A
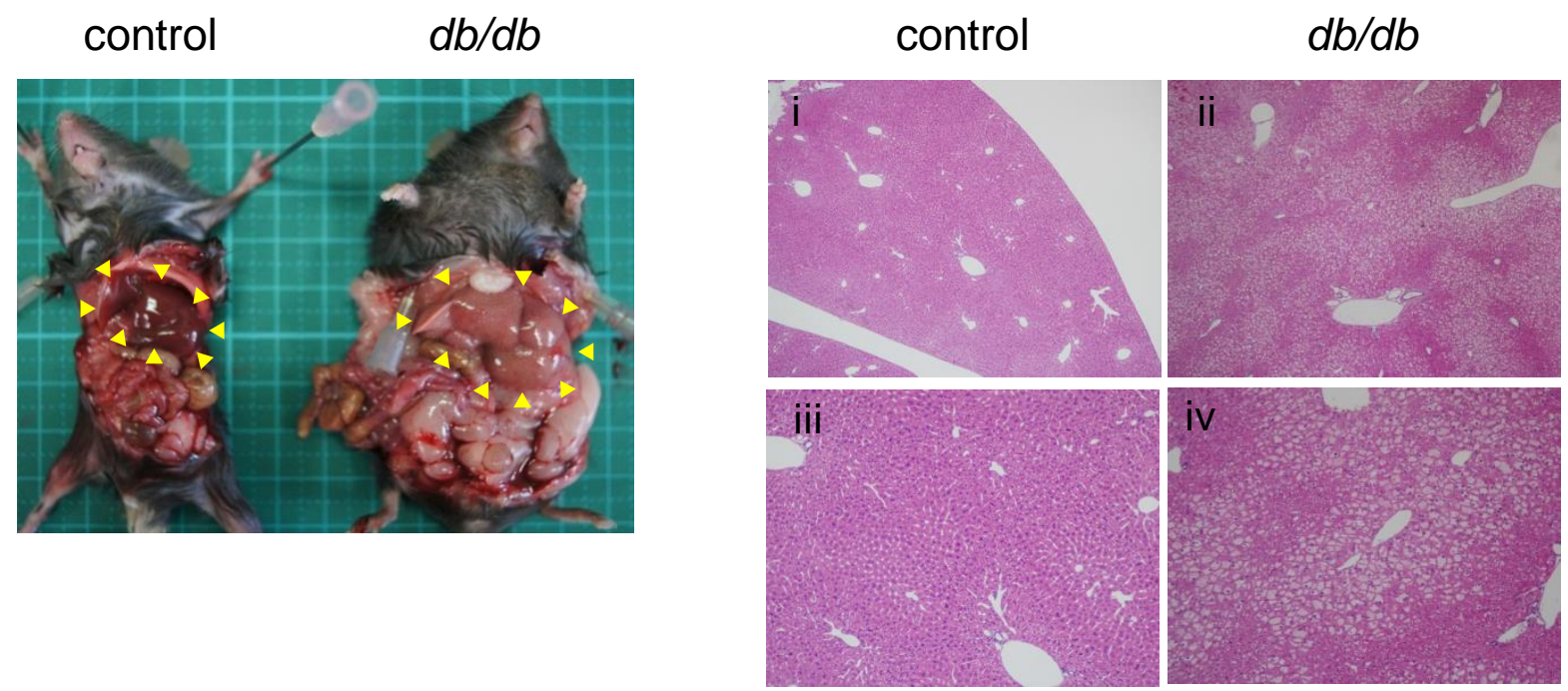

B

C
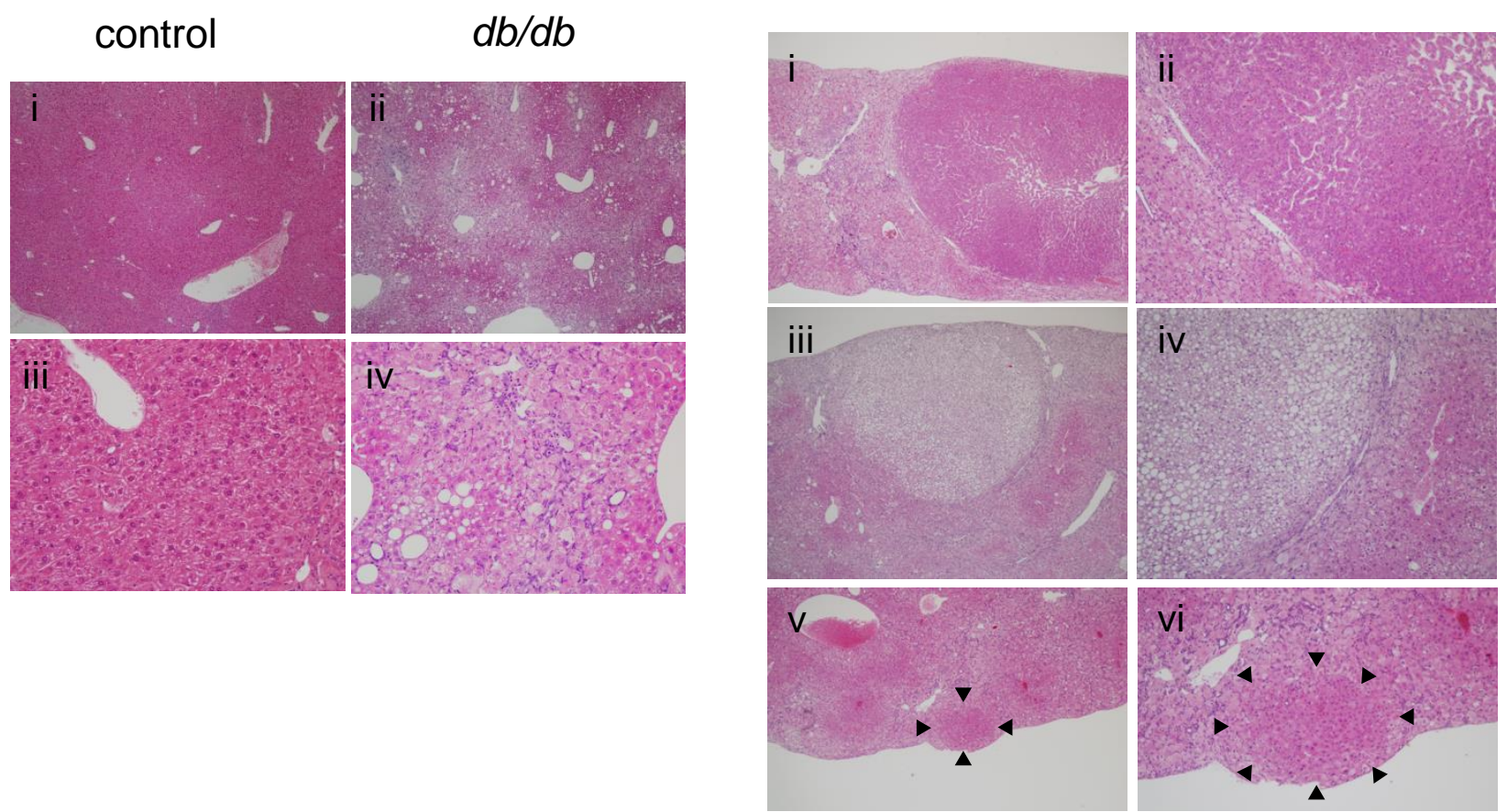